\title{
Mechanical spectroscopy of polymers with reduced dimensions and increasing cross-linking degree
}

\author{
Dissertation \\ zur Erlangung des Doktorgrades der Mathematisch-Naturwissenschaftlichen Fakultäten \\ der Georg-August-Universität zu Göttingen
}

Vorgelegt von

Tatjana Haramina, geb. Sušić

aus Karlovac, Kroatien

Göttingen 2006 
D7

Referent: Prof. Dr. Reiner Kirchheim

Korreferent: Prof. Dr. Konrad Samwer

Tag der mündlichen Prüfung: 27.06.2006 


\section{Contents}

1 Introduction $\quad 3$

2 Viscoelastic Behavior of Polymers 6

2.1 The mechanical response of matter . . . . . . . . . 6

2.2 Polymer glasses . . . . . . . . . . . . . . . . 7

2.2.1 Glassforming polymers . . . . . . . . . . . . 9

2.3 The experimental glass transition . . . . . . . . . . . 9

2.4 Some theoretical approaches for understanding the glass transition . . . . . . . . . . . . . . 11

2.4.1 Free volume theory . . . . . . . . . . . . . . 11

2.4 .2 The idea of cooperativity . . . . . . . . . 12

3 Vibrating Reed Technique $\quad 13$

3.1 The theory of flexural bending and vibrations . . . . . . . . 14

3.2 Damping and internal friction . . . . . . . . . . . 18

3.2.1 Dynamic mechanical behavior . . . . . . . . . . 18

3.2 .2 Damping of polymers . . . . . . . . . . . 20

3.2 .3 Damping of the substrate . . . . . . . . . . 20

3.2.4 Damping of the system substrate-film . . . . . . . 21

4 Experimental $\quad \mathbf{2 4}$

4.1 Vibrating Reed Apparatus (VRA) . . . . . . . . . . . 24

4.1.1 Silicon cantilevers and clamps . . . . . . . . . . 27

4.1 .2 Excitation . . . . . . . . . . . . . . . 34

4.1.3 The laser and the position sensitive detector (PSD) . 36

4.1.4 Controlling and measuring the temperature . . . . . 37

4.1.5 Vacuum system . . . . . . . . . . . . . . . 38

4.1 .6 Precision of the VRA . . . . . . . . . . 38

4.2 Spin-coating of films . . . . . . . . . . . . . 38

4.2 .1 Vacuum chucks . . . . . . . . . . . . 40

4.3 Focused Ion Beam (FIB) and Scanning Electron Microscopy

$(\mathrm{SEM}) \ldots \ldots \ldots \ldots \ldots \ldots \ldots$ 
5 Effect of Reduced Dimensions on the Glass Transition of Thin Polystyrene Films $\quad 42$

5.1 Introduction . . . . . . . . . . . . . . . . 42

5.1.1 The influence of confinement on dynamics . . . . . . 45

5.1.2 The influence of the chain length on dynamics . . . 46

5.1.3 The influence of the heating (cooling) rate on dynamics 47

5.2 Spin-coating of thin films . . . . . . . . . . 47

5.3 Mechanical spectroscopy of thin PS films . . . . . . . 48

5.3.1 Mechanical spectra of thick polymer films . . . . . . 48

5.3.2 Mechanical spectra of thin polymer films . . . . . . 51

5.3.3 Effect of the film damping on the damping of the composite . . . . . . . . . . . . 57

5.3.4 Film thickness measured by FIB/SEM . . . . . . . . 59

5.4 Activation energy . . . . . . . . . . . . . . . . 60

5.5 Molecular weight distribution measured by Size Exclusive Chromatography $(\mathrm{SEC}) \ldots \ldots \ldots$. . . . . . . . . . 6 64

5.6 Discussion . . . . . . . . . . . . . . . . . 64

6 Mechanical Spectroscopy of Poly(vinyl cinnamate) with Different Cross-linking Degree $\quad 70$

6.1 Cross-linking . . . . . . . . . . . . . . . . 71

6.1.1 Cross-linking and the mechanical response . . . . . . 71

6.1.2 Light induced cross-linking . . . . . . . . . . 71

6.1.3 Cross-linking of poly(vinyl cinnamate) (PVCN) . . . 72

6.2 Cross-linking degree assessment by Fourier Transformed In-

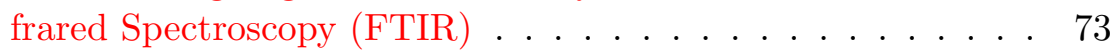

6.2.1 Preparation of samples for FTIR . . . . . . . . . 74

6.2.2 Infrared spectra depending on the UV irradiation time 74

6.3 Mechanical spectra depending on cross-linking degree . . . 78

6.3.1 Film preparation for VRA measurements . . . . . 78

6.3.2 Mechanical spectra . . . . . . . . . . . . 78

6.3.3 Eigen frequency influenced by cross-linking . . . . . . 82

6.3.4 Discussion on mechanical spectroscopy . . . . . . . . . 84

6.4 SEM Analysis . . . . . . . . . . . . . . . . 87

$\begin{array}{lll}7 & \text { Summary and outlook } & 89\end{array}$

A Energy-Dispersive X-Ray (EDX) Analysis of a Dry Etched Substrate. 


\section{Chapter 1}

\section{Introduction}

For over six decades it is known that many liquids which do not crystallize upon cooling undergo a transformation in many of their properties at a so-called glass transition temperature, $T_{g}$ (Bueche, 1953). From organic liquids to metals or polymers, almost any substance can be transformed into a glassy state. Above the $T_{g}$ these substances behave much like normal liquids and below they appear to be typical noncrystalline solids. The $T_{g}$ is for decades recognized to be the temperature at which the period of molecular motion approaches the time scale of the experiment (Bueche, 1953), the origins of the glass transition, however, despite its ubiquity in technology and everyday life still remain mysterious (Kawana and Jones, 2001). Though a fundamental parameter for describing a glass is the glass transition temperature, $T_{g}$, a more complete information about the dynamics at the near $T_{g}$ temperature range can be retrieved by mechanical dynamical measurements.

The mechanism and rate of conformational changes in polymer chains are mainly controlled by three factors: chain connectivity, intramolecular conformational potential and interactions with the environment (Bahar et al., 1995). The constrains imposed by chain connectivity operate on a wide spectrum of length scales, ranging from a few backbone bonds to Rouse type chain segments. The internal conformational potential of the chain may arise from bond stretching, distortion of bond angles and torsional rotations of bonds. The interaction with the environment are of either intermolecular or long-range intramolecular origin.

Dynamics of polymer chains may be observed by means of mechanical spectroscopy. The internal friction in the material, which is caused by conformational changes, is a function of a time lag between stress and strain, the anelasticity. The higher the time lag, the higher will be the damping of the material, hence the internal friction can be defined as a capacity of a material to damp out mechanical vibrations. The damping has its maximum when the period of molecular motions becomes comparable to the time scale of the experiment. Thus different relaxation processes lead to peaks in 
mechanical spectra.

In this work the mechanism of conformational changes and rate are influenced by cross-linking where the fixed cross-links hinder the mobility and by reduced dimensions where the ratio of the free surface area and the volume play a significant role in dynamics near the glass transition temperature. Both effects on structure of a polymer influence mechanical behavior of the material. Thus, the study on molecular dynamics reveals the problem of relating the structure with the materials properties. The mechanical spectroscopy measurements on polymer films with reduced dimensions and with increasing the cross-linking degree are performed by the vibrating reed method.

In recent years the finite size effects on glass transition was extensively investigated, but often with conflicting results, because the measurements were not performed in the same way. Three key factors influencing the observed depression of the glass transition temperature with reduced dimensions are found: the influence of a free surface, the influence of molecular weight and the third very important effect found by Fakhraai et al. (2005) is the influence of heating or cooling rate. The majority of measurements was performed by thermodynamic-type measurements and only a few dynamical studies on the effect of reduced dimensions are reported. These dynamical studies were performed by means of dielectric spectroscopy. The method allows investigation of the same sample at different frequencies, what is very important for observing the frequency dependent glass transition. However, the measured film is confined between two electrodes, without a free surface left. Therefore, the mechanical spectroscopy provides an alternative to the dielectric loss measurements. Mechanical spectroscopy of supported thin polymer films allows to study the effect of reduced dimensions on glass transition of polymer films with one free surface. So far there was not any report on dynamics of thin polymer films with at least one free surface. The measurements on polystyrene films within this work are performed with a low heating/cooling rate of $0.2 \mathrm{~K} / \mathrm{min}$. The material has high molecular weight of $1 \cdot 10^{6}$ and the polydispersity index of 1.06 . Therefore, all three demands for the proper investigation are fulfilled.

Mechanical spectroscopy of polymer films with increasing degree of crosslinking is performed on the example of poly(vinyl cinnamate) (PVCN). The cross-linked structure has a high influence on elastic modulus reflecting in damping properties of the material. The polymer is cross-linked by exposure to UV-light. This allows the study of the effect of cross-linking without using another substance as a cross-linker. The cross-linking with the UV light allows the control of the density of cross-links, by controlling the irradiation time. The structure and the density of the material is studied by the Fourier Transformed Infrared Spectroscopy (FTIR).

In Chapter 2 viscoelastic behavior of polymer glasses is described giving some basics about glass transition. Chapter 3 includes the mathematical 
description of flexural bending, vibrations and damping important for understanding the vibrating reed method. A description of the vibrating reed apparatus and the other equipment used within this work which is common for both experiments, the study of the effect of reduced dimensions and of the cross-linking effect, is given in Chapter 4. The methods and problems that are specific only for one experiment are given in Chapters referring to the respective experiments. In Chapter 5 the problem of the finite size effects and results on mechanical spectroscopy of polymer films are presented together with the FIB/SEM analysis of films. Chapter 6 deals with crosslinking of polymers the FTIR of cross-linked structure and the analysis of the effect of cross-linking on mechanical spectra of PVCN films. 


\section{Chapter 2}

\section{Viscoelastic Behavior of Polymers}

\subsection{The mechanical response of matter}

A standard condensed matter appears either as a solid or as a liquid. A stress applied on an ideal solid will produce a strain. The response is linear and elastic and the constant of proportionality E is called modulus (Hooke's law). Thus

$$
\sigma=E \epsilon,
$$

where $\sigma$ is the stress and $\epsilon$ is the strain. On the other hand the ideal liquids do not show elastic but irreversible viscous behavior and an applied stress produces a strain rate. The dependence is proportional and is given by the Newton's law

$$
\sigma=\eta \dot{\epsilon},
$$

where the coefficient $\eta$ is the viscosity.

Polymers are at the same time elastic and viscous and the common name for such a behavior is viscoelasticity. The response on the applied stress by viscoelastic materials is time dependent and if a stress is applied on a short timescale the material will behave like a solid while for longer times it exhibits viscous flow. The critical time between the solid like and the liquid like behavior is the relaxation time $\tau$. The approximate relationship between the viscosity $\eta$, an instantaneous elastic modulus $E_{0}$ and the relaxation time is given by

$$
\eta \sim E_{0} \tau .
$$

In simple liquids the relaxation times take values of order $10^{-12}-10^{-10} s$, what is short compared to typical experimental times, so they typically show 
purely viscous flow. In contrast, polymers have relaxation times of order milliseconds or even seconds. This range of values is easily discernible to the human senses, so their viscoelastic properties can be very spectacular (Jones, 2003).

The characteristic relaxation time $\tau$ depends on the energy barrier $Q_{0}$ (which is related to the latent heat of vaporisation per atom or molecule) that has to be overcome and the probability of overcoming is given by the Boltzmann distribution. Thus the characteristic time can be estimated as

$$
\tau^{-1} \sim \nu \exp \left(-\frac{Q_{a}}{k T}\right),
$$

where $\nu$ is comparable to the frequency of attempts to overcome the energy barrier, $k$ is the Boltzmann constant and $T$ is the temperature.

Putting together (Eq. 2.3) and (Eq. 2.4) the dependence of the viscosity of a simple liquid on the inverse temperature can be expressed by the Arrhenius equation:

$$
\eta=\frac{E_{0}}{\nu} \exp \left(\frac{Q_{a}}{k T}\right) .
$$

Experimental results for many liquids confirm this picture, though at low temperatures substantial deviations are found. This much stronger dependence of relaxation times on temperature is a result of the breakdown of the simple-atom picture of relaxation and leads to an entirely new phenomenon, the transition from a liquid to a glassy state. (Jones, 2003)

\section{$2.2 \quad$ Polymer glasses}

If a liquid is cooled down to a low enough temperature without crystallizing its relaxation time increases so strongly that it will be comparable with the experimental time scales. The liquid becomes a glass. In comparisson with the change in viscosity (or relaxation time) by heating the liquid, such a strong change by the glass transition is not expected (Jones, 2003). The reason for that is not clear and a proper physical description of the glass transition remains a key open question in condensed matter physics (Forrest and Sharp, 2003).

As mentioned in Section 2.1, at relatively high temperatures it is found that relaxation times or viscosity in liquids do depend on temperature according to the Arrhenius law. At low temperatures, assuming that the liquid has not crystallized, the situation is different. The relaxation time associated with changes in configuration of atoms or molecules, $\tau_{\text {config }}$, assumes a temperature dependence that strongly departs from the temperature dependence of the characteristic time of vibrations, $\tau_{v i b}$ (Fig. 2.1).

As the temperature is lowered, the state in which the relaxation time becomes comparable to the timescale of the experiment, $\tau_{\exp }$, is reached. When 


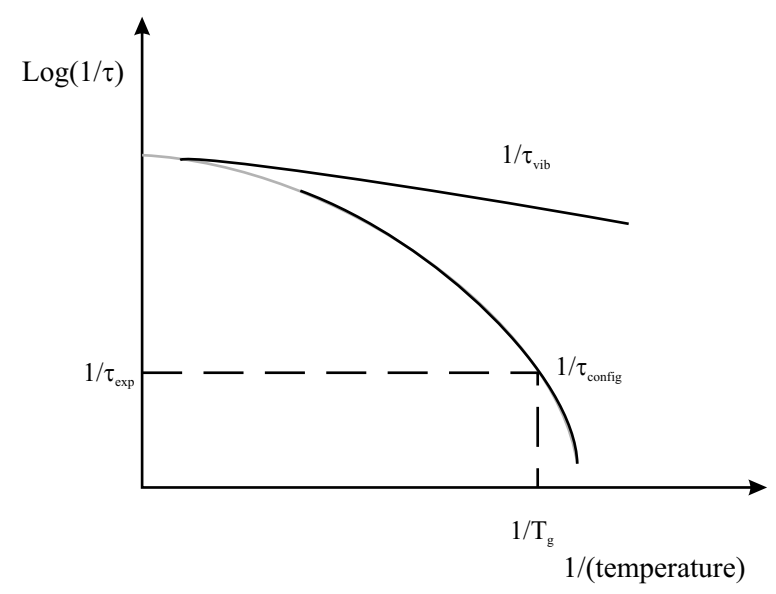

Figure 2.1: Temperature dependence of relaxation times in a glass-forming liquid (Jones, 2003).

this happens the system falls out of equilibrium with respect to configurational degrees of freedom and the structure appears frozen on the laboratory time scale. This marks the onset of the experimental glass transition.

Furthermore it is found that the configurational relaxation time appears to diverge at a finite temperature $T_{0}$, the Vogel-Fulcher-Tammann temperature. This temperature is in any case below the glass transition temperature and close to the Kauzmann temperature $T_{K}$, which marks a lower limit on the experimental glass transition temperature (see Section 2.3).

Experimentally, it is found that the temperature dependence of the relaxation time, and thus of the viscosity, close to the glass transition for silica is reasonably well described by the Arrhenius functionality (Eq. 2.5), while other liquids exhibit more pronounced viscous slow down following an empirical law known as the Vogel-Fulcher-Tamman (VFT) law:

$$
\eta=\eta_{0} \exp \left(\frac{B}{T-T_{0}}\right)
$$

where $\eta_{0}$ and $B$ are temperature independent constants (Jones, 2003), (Debenedetti and Stillinger, 2001). Since the VFT temperature $T_{0}$ means the structural arrest of a glass, the VFT law can be applied only for temperatures higher than $T_{0}$. At relatively high temperatures the VFT law turns into a simple Arrhenius law with the activation energy $Q_{a}$ (Buechenau, 1991).

Angell (1988) proposed a classification of liquids along the 'strong' and 'fragile' scale. The behavior of the former is nearly in the Arrhenius fashion, whereas that of the fragile shows marked deviations. Polymers are generally fragile and have relatively high viscosity at high temperatures because the disentaglement of long molecules lasts longer than the flow of substances of 


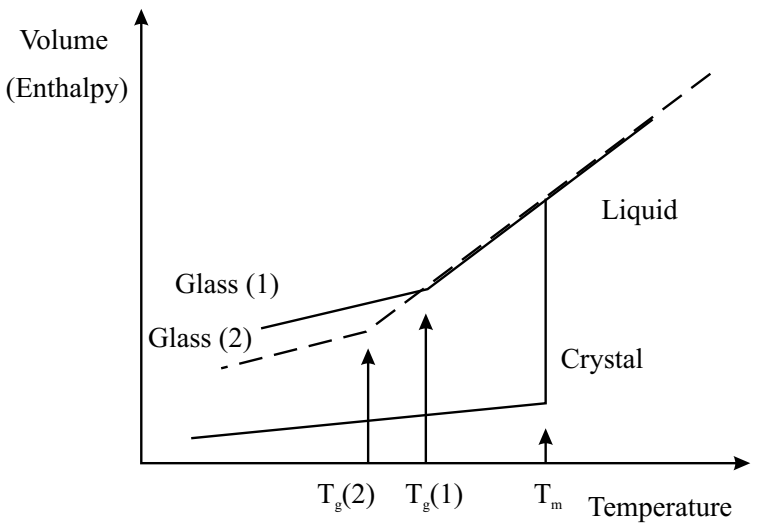

Figure 2.2: Volume (or enthalpy) changes upon cooling a glass-forming liquid. The slower the cooling rate is, the lower the $T_{g}$ will be.

low molecular weight (Buechenau, 1991).

\subsubsection{Glassforming polymers}

The major factor determining whether a polymer chain can crystallize is the occurrence of successive units in the chain in a configuration of high geometrical regularity. If the chain elements are small, simple and equal, as in linear polyethylene, crystallinity may be highly developed. If, however the chain elements are complex and contain bulky (side) groups, as in polystyrene and poly(vinyl cinnamate), the material can crystallize only if these substituent groups are arranged in an ordered or tactic configuration (Van Krevelen, 1990).

The polymers studied in this work are of the atactic arrangement and that involves quenched disorder. In atactic macromolecules every substituent belonging to a repeating unit is placed randomly at either side of the backbone. The energy barriers for rotation of the side groups are so large that once the arrangement is set in place during synthesis of the polymer, no rearrangement can take place (Jones, 2003). Hence, by cooling below the $T_{g}$, the systems studied in this work will form glasses.

\subsection{The experimental glass transition}

Fig. 2.2 illustrates the temperature dependence of a liquid's volume (or enthalpy) at constant pressure. If the liquid is able to form a crystal, it will crystallize at some well defined temperature $T_{m}$. However, under the circumstances described in Section 2.2 it is possible to cool a liquid down below its freezing point without it crystallizing. This may be because the cooling rate is so fast that the liquid does not have time to crystallize, 
or it may be because the molecules have some permanent disorder which inhibits them from forming crystals at all. Whatever the reason, at some temperature there will be a change in slope of the graph, corresponding to a discontinuous change in the thermal expansivity (see 'Glass (1)' curve in Fig. 2.2). This discontinuity marks the glass transition temperature $T_{g}$.

The glass transition is similar in appearance to a second-order phase transition, but it is not a true thermodynamic phase transition. This is because the transition temperature depends on the rate at which an experiment is performed. If $\tau_{\text {exp }} \approx \tau_{\text {config }}$ (Fig. 2.1), the degrees of freedom associated with translational motion of the molecules can no longer come to equilibrium on the timescale of the experiment. Because of this, these degrees of freedom can no longer contribute to the values of thermodynamic quantities as they are measured in the experiment and a glass transition is observed.

If the experiment is done more slowly $\tau_{\exp }$ will be increased so $\tau_{\exp } \approx$ $\tau_{\text {config }}$ will be at a lower temperature and as depicted in Fig. 2.2 the 'Glass (2)' curve with $T_{g}(2)<T_{g}(1)$ will be observed.

By means of the calorimetric measurements at constant pressure, the heat capacity $c_{p}$ can be measured. It is related to the entropy of the system by the relation:

$$
c_{p}=T\left(\frac{\partial S}{\partial T}\right)_{p} .
$$

By integrating experimental heat capacity curves, the entropy as a function of temperature can be found. Qualitatively are the graphs (Fig. 2.3) similar to those in a plot of volume against temperature (Fig. 2.2). Here one also observes the experimental glass transition depending on the cooling rate. The entropy of a glass is not a pure thermodynamic function of state, because it depends not only on temperature and pressure, but also on the history of the sample. By decreasing the cooling rate, lower and lower glass transitions are expected. Figure 2.3 shows that at a certain temperature the extrapolation of the entropy curve for the supercooled liquid intersects the entropy curve for the crystalline solid. Since it does not make physical sense for a glass to have a lower entropy than the crystal, it is suggested that this temperature, which is known as the Kauzmann temperature $T_{K}$, marks an effective lower limit on the experimental glass transition temperature. Estimates of the Kauzmann temperature reveal that it is often remarkably close to the Vogel-Fulcher temperature $T_{0}$.

Despite the similarity to the second-order phase transition (the transition between liquid state and a glass is marked by discontinuities in thermodynamic quantities that are second derivatives of a free energy), the glass transition should be understood as a kinetic transition, since its location depends on the rate at which the experiment was carried out. Thus the 


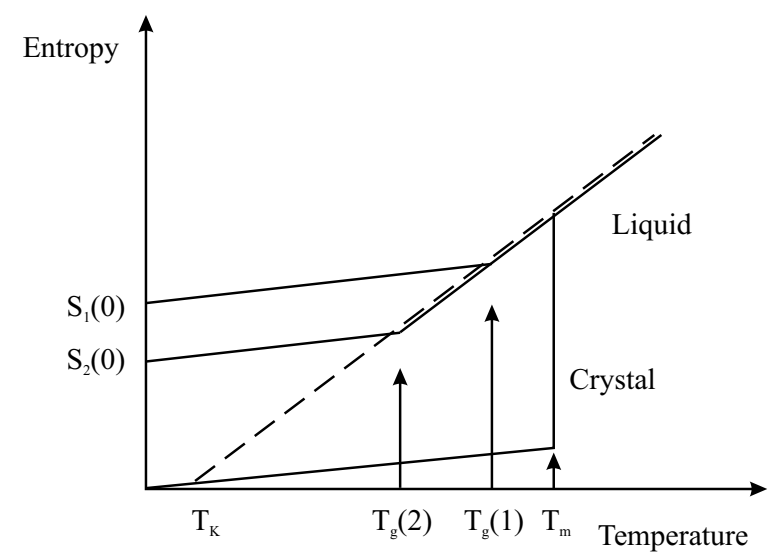

Figure 2.3: Entropy changes on cooling a glass-forming liquid. $T_{g}(2)$ represents the experimental glass transition observed using lower cooling rate than $T_{g}(1)$.

glassy state is not an equilibrium phase, it is not the state of the lowest free energy.

\subsection{Some theoretical approaches for understand- ing the glass transition}

There are a variety of theoretical approaches, still they do not give a consistent and complete picture of the glass transition. Yet, they do give some insight into the origins of the glassy state. Here a brief description of the free volume theory which is an early approach but still popular in polymer science (Bueche, 1953) will be given and the idea of cooperativity which is useful in constructing theories of the glass transition. A description of some more recent approaches like Mode coupling theories or Theory of hierarchically correlated molecular motions are not given here. An introduction to these theories can be found in e.g. (Cavaille et al., 2001).

\subsubsection{Free volume theory}

The main assumption in this theory is that a liquid of a volume $v$ contain a certain amount of free volume $v_{f}$, which is available to permit motion of nearby segments. It is argued that the fractional free volume $v_{f} / v$ can be written as a simple linear function of temperature, as

$$
\frac{v_{f}}{v}=f_{g}+\alpha_{f}\left(T-T_{g}\right),
$$

where $f_{g}$ is the fractional free volume at the glass transition temperature $T_{g}$, and $\alpha_{f}$ is the expansion coefficient of the free volume. If there is a simple relationship between free volume and viscosity $\eta$ of the form 


$$
\eta=a \exp \left(\frac{b v}{v_{f}}\right),
$$

where $a$ and $b$ are constants, then the VFT relationship can be recovered.

One drawback of the theory are the rather arbitrary assumptions for Eq. 2.8 that make the theory not really predictive. Another one is that not all the predictions that the theory does make are confirmed by experiment (Jones, 2003).

\subsubsection{The idea of cooperativity}

Adam and Gibbs (1965) proposed that at high temperatures the space required for a molecule to move out of its location can be made by the uncoordinated, local vibrational motion of its neighbors and that at low temperatures this local motion is not sufficient, but a number of its neighbors must move cooperatively in order to make space. They introduced the concept of a cooperatively rearranging region to describe the minimum number of molecules that have to move cooperatively in order for any motion to take place. The size of the region becomes infinite at the VFT temperature where the viscosity diverges.

The activation energy is dependent on the number of molecules that have to move cooperatively, what explains the non-Arrhenius behavior of a glass as the temperature decreases. 


\section{Chapter 3}

\section{Vibrating Reed Technique}

As described in Chapter 2, viscoelastic materials can behave either elastically or viscously, depending on the timescale over which they are deformed; therefore experiments based on an oscillatory deformation at a certain frequency $\omega$ are extremely important for characterizing viscoelastic properties. Different relaxation processes have different relaxation times. Thus, in order to learn more about these relaxation processes, mechanical spectroscopy methods of time scales comparable to the respective relaxation times have to be developed.

The vibrating reed technique is a mechanical spectroscopy method by which the free vibration of a bar is measured in a given temperature range. The more internal friction, the higher will be the damping. At low temperatures the motion of molecules is too slow to contribute to the damping and at high temperatures it is too fast. The movements, the relaxation times of which are comparable to the experimental time scale lead to peaks in the mechanical spectra.

In this chapter the equations describing vibrations and the damping of a clamped bar used in this work are presented. In Section 3.1, a mathematical description of flexural bending is given with the analysis of vibrations with the eigenfrequency for the system of a rectangular substrate with a thin film on it. The information obtained from the analysis is how to calculate the thickness of the pure substrate and how to figure out the film thickness from the measurements of a composite (substrate with a film on it). Additionally it is pointed out how the change in the elastic modulus of the film can be characterized by observing the frequency shift of the composite system. In Section 3.2 the equations related to the energy loss and the damping are presented with the analysis of main causes for the damping of vibrations. Here the analysis of the damping of the pure substrate is presented as well as that of the composite substrate-film. For this work it is important to show how to calculate the internal friction of the film from the data measured for the composite. 


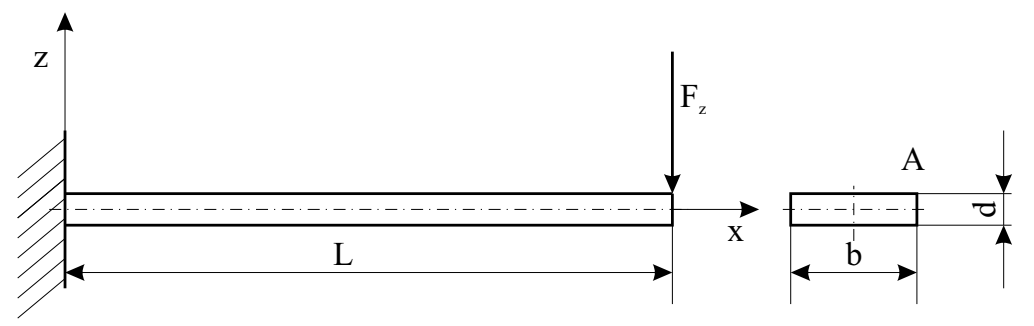

Figure 3.1: A reed of a cross section A, clamped on one side, with a force applied on a free end.

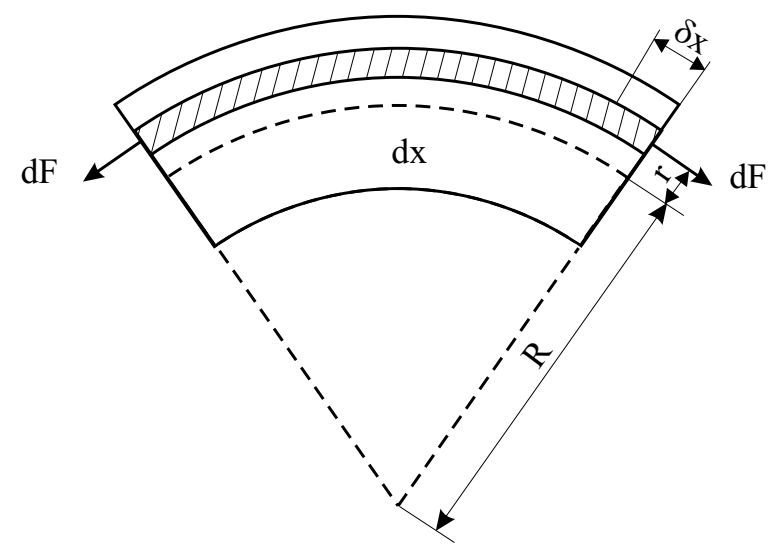

Figure 3.2: Bending strains and stresses in a bar (Kinsler and Frey, 1962).

\subsection{The theory of flexural bending and vibrations}

A straight bar of length $l$, having a uniform cross section area $A$, which is symmetric about a vertical plane through the axis of the bar is clamped on one side (Fig. 3.1). The bar is made of an elastic homogeneous material. The $x$ coordinate gives the position along the bar and the $z$ coordinate the transverse displacement of the bar from its normal configuration.

If a force is applied on the free end, the bar will bend. Here the bending of the console as a function $z(x)$ is calculated with an assumption that the local curvature radius remains much smaller than that of the bar; therefore the plastic deformation can be neglected. It is obvious that the material on the upper part of the console will be stretched and contrary on the lower side it will be compressed. Between these two areas there must be a neutral axis, the length of which will not change in the case of elastic deformation. The local $z$ axis has its origin starting from the neutral axes as depicted in Fig. 3.1. The local strain is then

$$
\epsilon_{x}=\frac{\Delta l}{l}=\frac{(R+r) \theta-R \theta}{R \theta}=\frac{r}{R} .
$$

A general analytical expression for the radius of curvature, $R$, is 


$$
\frac{1}{R}=\frac{\partial^{2} z / \partial x^{2}}{\left(1+(\partial z / \partial x)^{2}\right)^{3 / 2}},
$$

and for $d z / d x<<1$ the approximate relation may be used

$$
\frac{1}{R} \approx \frac{\partial^{2} z}{\partial x^{2}} .
$$

The total longitudinal force $F_{x}=\int d F$ is zero, negative forces above the neutral axis being canceled by positive forces below the neutral axis. However, a bending moment $\mathrm{M}$ is present in the bar. The moment is given by

$$
M=-\int_{A} r d F=-\int_{A} r \sigma_{x} d A .
$$

Combined with Eqs. 2.1 and 3.1 the moment can be expressed as

$$
M=-\int_{A} E \frac{r^{2}}{R} d A .
$$

For a bar of a rectangular cross section consisting of a substrate and a thin film applied on it, the moment can be calculated as

$$
-M=\int_{-d_{s} / 2}^{d_{s} / 2} E_{s} \frac{r^{2}}{R} b d z+\int_{d_{s} / 2}^{d_{s} / 2+d_{f}} E_{f} \frac{r^{2}}{R} b d z,
$$

where $\mathrm{b}$ is the width, $d_{s}$ and $d_{f}$ are the thicknesses of the substrate and of the film, respectively and $E_{s}$ and $E_{f}$ are the moduli of the substrate and of the film, respectively.

The integration gives

$$
-M=\frac{E_{s} b d_{s}^{3}}{12 R}+\frac{E_{f} b d_{s}^{2} d_{f}}{4 R}=\frac{b d_{s}^{3}}{12 R}\left(E_{s}+\frac{3 E_{f} d_{f}}{d_{s}}\right) \equiv \frac{b d_{s}^{3}}{12 R} E_{c}^{*} .
$$

Including Eq. 3.3 into Eq. 3.7 yields

$$
-M=\frac{b d_{s}^{3}}{12} E_{c}^{*} \frac{\partial^{2} z}{\partial x^{2}} \equiv D_{c} \frac{\partial^{2} z}{\partial x^{2}} .
$$

The effect of distorting the bar is to produce not only bending moments but also shear forces (Fig. 3.3).

For small displacement the variations in angular momentum may be neglected; therefore, from the sum of moments about the left end of the segment, the shear force $F_{z}$ can be expressed as $-\partial M / \partial x$ (Kinsler and Frey, 1962). The net upward force $d F_{z}$ acting on the segment $d x$ is then given by 


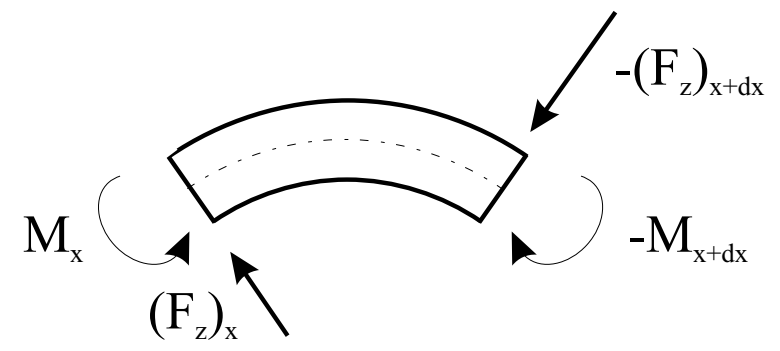

Figure 3.3: Moments and shear forces produced by distorting the bar.

$$
d F_{z}=D_{c} \frac{\partial^{4} z}{\partial x^{4}} .
$$

This force will give the segment an upward acceleration. It is in a balance with

$$
d F(x)=\frac{d^{2} z}{d t^{2}} d m
$$

Since the mass of the segment $\mathrm{dm}$ is $b\left(\rho_{s} d_{s}+\rho_{f} d_{f}\right) d x \equiv \mu_{c} d x$, the equation of motion can be written as

$$
\mu_{c} \frac{\partial^{2} z}{\partial t^{2}}=D_{c} \frac{\partial^{4} z}{\partial x^{4}}
$$

This differential equation can be solved by the method of separation of variables. The solution for the displacement $z$ is then

$$
\mathbf{z}=e^{i \omega t}\left(\mathbf{A} e^{\omega x / v}+\mathbf{B} e^{-\omega x / v}+\mathbf{C} e^{i \omega x / v}+\mathbf{D} e^{-i \omega x / v}\right),
$$

where $v=\sqrt{\omega c \kappa}$, c is defined as $\sqrt{E / \rho}$ and $\kappa$ is a constant depending on the cross section. For the rectangular cross section $\kappa=d / \sqrt{12}$ (Kinsler and Frey, 1962).

If one end of the bar is rigidly clamped, both the displacement and the slope must be zero at this end for all times $t$. The boundary conditions for the clamped end are therefore $z=0$ and $\partial z / \partial x=0$.

At the free end both $M$ and $F_{z}$ are zero in a plane located at an infinitesimal distance from the end perpendicular to the $x$ axis, hence $\partial^{2} z / \partial x^{2}=0$ and $\partial^{3} z / \partial x^{3}=0$.

This gives

$$
\cosh \frac{\omega l}{v} \cos \frac{\omega l}{v}=-1 .
$$

According to e.g. Kinsler and Frey (1962) the frequencies corresponding to the allowed modes of vibration are given by 


$$
\frac{\omega l}{2 v}=\frac{\pi}{4}(1.194,2.988,5,7, \ldots) .
$$

In the experiment done within this work only the first mode vibrations are excited. For the first mode follows the eigenfrequency of the composite, film on a substrate,

$$
f_{c}=1.194 \frac{\pi c \kappa}{8 l^{2}}=\frac{0.1615}{l^{2}} \sqrt{\frac{D_{c}}{\mu_{c}}} .
$$

or

$$
f_{c}=\frac{0.1615}{l^{2}} \sqrt{\frac{b d_{s}^{3} E_{s}\left(1+3 E_{f} d_{f} / E_{s} d_{s}\right)}{12 b \rho_{s} d_{s}\left(1+\rho_{f} d_{f} / \rho_{s} d_{s}\right)}} .
$$

Since the systems observed consist of a silicon substrate and a thin polymer film, both the thickness and the modulus of the film are much smaller than those of the substrate. This allows to neglect of higher derivatives in the Taylor series giving

$$
f_{c} \approx \frac{0.1615}{l^{2}} \sqrt{\frac{d_{s}^{2} E_{s}}{12 \rho_{s}}}\left(1+\frac{3 E_{f} d_{f}}{2 E_{s} d_{s}}\right)\left(1-\frac{\rho_{f} d_{f}}{2 \rho_{s} d_{s}}\right) .
$$

With the frequency of the pure substrate

$$
f_{s}=\frac{0.1615 d_{s}}{l^{2}} \sqrt{\frac{E_{s}}{\rho_{s}}} .
$$

substituted into Eq. 3.17 the frequency of the composite can be expressed as

$$
f_{c} \approx f_{s}\left(1+\frac{3 E_{f} d_{f}}{2 E_{s} d_{s}}\right)\left(1-\frac{\rho_{f} d_{f}}{2 \rho_{s} d_{s}}\right) \approx f_{s}\left(1+\frac{3 E_{f} d_{f}}{2 E_{s} d_{s}}-\frac{\rho_{f} d_{f}}{2 \rho_{s} d_{s}}\right) .
$$

The difference between Eqs. 3.19 and 3.18 gives an expression for calculating the thickness of polymer films from the frequency shift $\Delta f=f_{c}-f_{s}$ caused by the increase in mass

$$
\frac{\Delta f}{f_{s}} \approx\left(\frac{3 E_{f}}{2 E_{s}}-\frac{\rho_{f}}{2 \rho_{s}}\right) \frac{d_{f}}{d_{s}} .
$$

The thicker the film, the lower the resonant frequency.

For a rough estimation of the film thickness, since $E_{f}<<E_{s}$, the thickness can be calculated as

$$
\frac{\Delta f}{f_{s}} \approx-\frac{\rho_{f}}{2 \rho_{s}} \frac{d_{f}}{d_{s}}
$$


For the case of cross-linking of the polymer film (Chapter 6), when the modulus of a material changes, but the product $\rho_{f} d_{f}$ remains constant, the eigenfrequency changes, too. This allows the change in the modulus to be calculated from the measured frequency shifts

$$
\frac{\Delta \Delta f}{f_{s}}=\frac{\Delta f-\Delta f_{(x)}}{f_{s}}=\frac{3}{2 E_{s} d_{s}}\left(E_{f} d_{f}-E_{f x} d_{f x}\right),
$$

where $\Delta f$ is the difference in the eigenfrequency of the substrate and an uncross-linked film and $\Delta f_{(x)}$ is the difference in the eigenfrequency of the substrate and the cross-linked film.

If we suppose that the film thickness does not change significantly with increasing cross-link density then

$$
\frac{\Delta \Delta f}{f_{s}}=\frac{3 \Delta E_{f} d_{f}}{2 E_{s} d_{s}} .
$$

\subsection{Damping and internal friction}

\subsubsection{Dynamic mechanical behavior}

The experiments based on an oscillatory deformation at a certain frequency $\omega$ are very powerful measurements for obtaining information on time dependent viscoelastic behavior.

If an oscillatory stress $\sigma$ of the form

$$
\sigma=\sigma_{0} \exp (i \omega t)
$$

is applied then for small amplitudes the resulting strain $\epsilon$ will lag behind the stress for a phase angle $\phi$. This can be written as

$$
\epsilon=\epsilon_{0} \exp [i(\omega t-\phi)] .
$$

$\sigma_{0}$ and $\epsilon_{0}$ are stress and strain amplitudes respectively.

The ratio $\sigma / \epsilon$ is a complex quantity, called complex modulus $E^{*}$ and is a function of the circular frequency $\omega$. The complex modulus can be then written as

$$
E^{*}=E^{\prime}+i E^{\prime \prime},
$$

where the real part, $E^{\prime}$, is known as the storage modulus and the imaginary part, $E^{\prime \prime}$, is known as the loss modulus. The phase angle of the response is given by

$$
\tan \phi=E^{\prime \prime} / E^{\prime}
$$

and the modulus $\mathrm{E}$ is given by 


$$
E=|E|=\sqrt{\left(E^{\prime}\right)^{2}+\left(E^{\prime \prime}\right)^{2}} .
$$

The ratio of energy dissipated per cycle and the mean energy of the system between two successive vibrations is commonly called the quality factor $Q^{-1}$. It is a measure of the internal friction in a solid (Zener, 1937). Hence

$$
Q^{-1}=\frac{1}{2 \pi} \frac{\Delta W}{W} .
$$

In the case where $\phi<<1$, the following approximations are valid (Nowick and Berry, 1972)

$$
\tan \phi \approx \phi \approx Q^{-1} \approx \frac{\lambda_{D}}{\pi},
$$

where $\lambda_{D}=\delta T=\delta / f$ is the logarithmic decrement, $\delta$ is the damping factor and $\mathrm{T}$ is the period. The approximations are valid to within $1 \%$ of the exact value up to $\tan \phi=0.2$ (Graeser and Wong, 1992).

From 3.27, 3.28 and 3.30 the storage and the loss modulus follow respectively:

$$
E^{\prime}=\frac{E}{\sqrt{1+\left(Q^{-1}\right)^{2}}}
$$

and

$$
E^{\prime \prime}=\frac{Q^{-1} E}{\sqrt{1+\left(Q^{-1}\right)^{2}}} .
$$

The modulus is frequency dependent; therefore there are two extreme cases, the relaxed modulus $M_{R}$ (low frequencies) and the unrelaxed modulus $M_{U}$ (high frequencies). It is convenient to define a dimensionless quantity $\Delta$, called a relaxation strength, by

$$
\Delta=\frac{M_{U}-M_{R}}{M_{R}} .
$$

For $\Delta<<1$, the equation for the internal friction may also be expressed as a Debye function

$$
Q^{-1} \approx \Delta \frac{\omega \tau}{1+\omega^{2} \tau^{2}}
$$

The function has its maximum in $\omega \tau=1$ and is valid only for the systems with only one relaxation time $\tau$. However, in a real solid different processes cause the anelasticity. This leads to a spectrum of the relaxation times and hence a broadening of the relaxation peak. 


\subsubsection{Damping of polymers}

The damping of polymers is a consequence of their viscoelastic nature (Chapter 2). The mechanical response that we observe at the macroscopic level can be understood at the microscopic level in terms of the role of bond energies and timescales of segments and molecular motions.

In this the glass transition is observed. The origin of the glass transition is mostly in long-range segmental motion. A polymer with a backbone that exhibits higher flexibility will have a lower $T_{g}$. This is because the activation energy for conformational changes is lower. Since the relaxation times of different segments differ from each other, the relaxation peak is broader than the Debye peak. Additionally, for polystyrene at $f>40 \mathrm{~Hz}$ it is known (McCrum et al., 1991) that slow $\beta$ relaxation merges with the $\alpha$ relaxation (or glass transition). The relaxation strength, which is to observe in the peak height, increases with the increase in the film thickness. In the experiments performed within this work the $\alpha$ relaxation is influenced by reducing the thickness of the polymer film and by cross-linking the chains.

\subsubsection{Damping of the substrate}

The damping of the single crystal silicon substrate is mainly caused by thermal relaxation. The two sides of a reed vibrating transversely have dilations of opposite signs. In an isotropic solid with a positive thermal expansion coefficient, a positive dilation lowers the temperature and a negative dilation raises the temperature. Therefore an alternating temperature gradient exists between the two faces of the reed. Relaxation can thus occur by heat flow from the compressed to the extended layers.

The thermoelastic damping shows the Debye behavior described with Eq. 3.34 with the relaxation strength

$$
\Delta=E \alpha^{2} T / c_{p}
$$

therefore

$$
Q_{t h}^{-1} \approx \frac{E \alpha^{2} T}{c_{p}} \frac{\omega \tau}{1+\omega^{2} \tau^{2}},
$$

with the relaxation time

$$
\tau=\frac{d_{s}^{2} c_{p}}{\pi^{2} \lambda_{t h}},
$$

where $\alpha$ is the thermal expansion coefficient, $c_{p}$ is the specific heat per unit volume, $\mathrm{T}$ is the temperature and $\lambda_{t h}$ is the thermal conductivity ((Nowick and Berry, 1972)).

Since Si has a very small thermal expansion, the relaxation strength of the reed is also very small. With $c_{p}=1.66 \cdot 10^{6} \mathrm{~J} / \mathrm{m}^{3} \mathrm{~K}, \alpha=2.6 \cdot 10^{-6}$ 
$\mathrm{m} / \mathrm{mK}$ and $\lambda_{t h}=150 \mathrm{~W} / \mathrm{mK}$, the relaxation strength for the reeds used in the experiments described in Chapter $4\left(d_{s}\right.$ between 30 and $\left.100 \mu \mathrm{m}\right)$ is between $10^{-5}$ and $10^{-4}$, depending on the reed, the relaxation time between $10^{-6}$ and $10^{-5} \mathrm{~s}$ and the thermal damping between $10^{-6}$ and $10^{-5}$. The exact value for each type of read will be calculated in Chapter 4 .

Inserting Eqs. 3.18 and 3.37 into Eq. 3.34 (and neglecting the denominator of Eq. 3.34, since $\tau^{2} \approx 10^{-10}$ ) gives the dependence of thermal damping on dimensions of a reed

$$
Q_{t h}^{-1} \propto \frac{d_{s}^{3}}{L^{2}},
$$

where $\mathrm{L}$ is the length of the reed. The thermal damping is independent on the width of the reed. This is a very useful equation for choosing substrates of minimal damping.

In order to prevent oxidizing and reduce friction with the gas molecules, the measurements are performed in vacuum of about $10^{-6}$ mbar. According to Newell (1968) damping of a rectangular reed in a gas atmosphere can be calculated as

$$
Q_{\text {gas }}^{-1}=\left(\frac{2}{\pi}\right)^{3 / 2} \sqrt{\frac{M}{R T}} \frac{1}{\rho_{s} d_{s}} \frac{p}{f},
$$

where $\mathrm{M}$ is the molecular mass of the gas molecules, $\mathrm{R}$ is the universal gas constant, $\mathrm{T}$ is the temperature and $\mathrm{p}$ is the pressure. With $M=29$ $\mathrm{g} / \mathrm{mol}$ (air), $R=8.314 \mathrm{~J} / \mathrm{Kmol}, T=300 \mathrm{~K}, \rho=2330 \mathrm{~kg} / \mathrm{m}^{3}, d_{s}=100$ $\mu \mathrm{m}, p=10^{-4} \mathrm{~Pa}$ and $f=600 \mathrm{~Hz}$, the air damping is $Q_{\text {qas }}^{-1} \approx 10^{-9}$. For the thinner reeds and lower frequencies it can be up to $10^{-8}$.

Some additional damping comes from the friction in clamps. The experimental solutions for this problems are described in Chapter 4.

The single crystal silicon reeds used in this work are slightly phosphorus or boron doped in order to conduct the current necessary for the capacitive excitation. It is expected that there are also some other defects in the single crystal wafers. This causes some small additional internal friction.

\subsubsection{Damping of the system substrate-film}

Consider a system containing a silicon substrate and a thin polymer film applied on it. The internal friction of the film, the substrate and the composite of these two follow from Eq. 3.29. Hence

$$
\begin{aligned}
Q_{f}^{-1} & =\frac{1}{2 \pi} \frac{\Delta W_{f}}{W_{f}}, \\
Q_{s}^{-1} & =\frac{1}{2 \pi} \frac{\Delta W_{s}}{W_{s}}
\end{aligned}
$$


and

$$
Q_{c}^{-1}=\frac{1}{2 \pi} \frac{\Delta W_{c}}{W_{c}} .
$$

Since the sum of the energies must remain constant, the following equations are valid:

$$
W_{c}=W_{s}+W_{f}
$$

and

$$
\Delta W_{c}=\Delta W_{s}+\Delta W_{f} .
$$

Substitution of Eqs. 3.40, 3.41 and 3.42 into Eq. 3.44 gives

$$
Q_{c}^{-1}=\frac{Q_{s}^{-1}+W_{f} Q_{f}^{-1} / W_{s}}{1+W_{f} / W_{s}} .
$$

The stored energy of the polymer film is much smaller then that of the silicon substrate $\left(\left(W_{f} / W_{s}\right)^{2} \approx 0\right)$; therefore

$$
\begin{aligned}
Q_{c}^{-1} & \approx\left(Q_{s}^{-1}+Q_{f}^{-1} \frac{W_{f}}{W_{s}}\left(1-W_{f} / W_{s}\right)\right) \approx \\
& \approx Q_{s}^{-1}+Q_{f}^{-1} \frac{W_{f}}{W_{s}}-Q_{s}^{-1} \frac{W_{f}}{W_{s}} .
\end{aligned}
$$

The difference between the $Q^{-1}$ factors of the composite sample and that of the pure substrate is then

$$
\Delta Q^{-1}=Q_{c}^{-1}-Q_{s}^{-1}=\frac{W_{f}}{W_{s}}\left(Q_{f}^{-1}-Q_{s}^{-1}\right) \approx \frac{W_{f}}{W_{s}} Q_{f}^{-1}
$$

The energy per length unit is

$$
d W_{s}=d x \int_{-d_{s} / 2}^{d s / 2} \frac{E_{s}}{2}\left(\frac{r-r_{0}}{R}\right)^{2} b d r .
$$

For neutral axis being at $r_{0} \approx 0$, substituting Eq. 3.3

$$
\begin{aligned}
d W_{s} & =\frac{E_{s}}{2}\left(\frac{d^{2} z}{d x^{2}} b d x\right)^{2} b d x \int_{-d_{s} / 2}^{d_{s} / 2}\left(r-r_{0}\right)^{2} d r= \\
& =\frac{E_{s}}{6}\left(\frac{d^{2} z}{d x^{2}} b d x\right)^{2} b d x\left(\frac{d_{s}^{3}}{4}+\frac{3}{2} d_{s} y_{0}^{2}\right)
\end{aligned}
$$

and 


$$
d W_{f}=d x \int_{-d_{s} / 2}^{d_{s} / 2+d_{f}} \frac{E_{f}}{2}\left(\frac{r-r_{0}}{R}\right)^{2} b d r \approx \frac{E_{f}}{2}\left(\frac{d^{2} z}{d x^{2}}\right)^{2} b d x \frac{d_{s}^{2}}{4} d_{f} .
$$

Substitution of these two equations into 3.47 with $r_{0}=0$

$$
\Delta Q^{-1}=\frac{3 E_{f} d_{f}}{E_{s} d_{s}} Q_{f}^{-1}
$$

From measurements got by means of the vibrating reed technique, $\Delta Q^{-1}$ is very obvious. The Eq. 3.51 allows the calculation of the internal friction of thin polymer films. 


\section{Chapter 4}

\section{Experimental}

The dynamical properties of polymer films depending on temperature are measured by means of the vibrating reed technique. A design of the apparatus was not a part of this work, but of the diploma thesis of Hölck (2000). In the frame of this work the apparatus is adapted for measurements on supported polymer films. Therefore in Section 4.1 the emphasis is put on assemblies (i.e. clamped substrate and film) that allowed observing the relaxation processes in thin films. The section includes a description of the vibrating system: the cantilevers, their respective clamps, the excitation and the detection of the vibration. Additionally a short description of the temperature regulation and of the vacuum system is given. Section 4.2 deals with spin-coating of films on substrates of different dimensions. Eventually, in Section 4.3 the microscopic methods applied for characterization of films, focused ion beam (FIB) and scanning electron microscopy (SEM), are presented.

The experimental part which is characteristic only for cross-linking and its characterization (the UV-lamp and the FTIR) is given in Chapter 6 .

\subsection{Vibrating Reed Apparatus (VRA)}

Vibrating reed technique is one of several resonance mechanical spectroscopy methods. By means of the VRA one can measure the internal friction in free standing bulk samples as well as in supported thin polymer films. The method is well established for nondestructive characterization of metal layers, but it is not as spread for the analysis of dynamics in thin polymer films. Thus e.g. Mizubayashi et al. (1992) and Harms et al. (1999) studied the elasticity of thin aluminum films, Tanimoto et al. (2004) studied the anelasticity of nanocrystalline metals or Hasegawa et al. (2004) dealt with hydrogen-induced internal friction of Zr-based bulk glassy alloys. On the other hand the relaxation processes in polymer films are mostly studied by dielectric spectroscopy. One of the rare works on mechanical spectroscopy of 


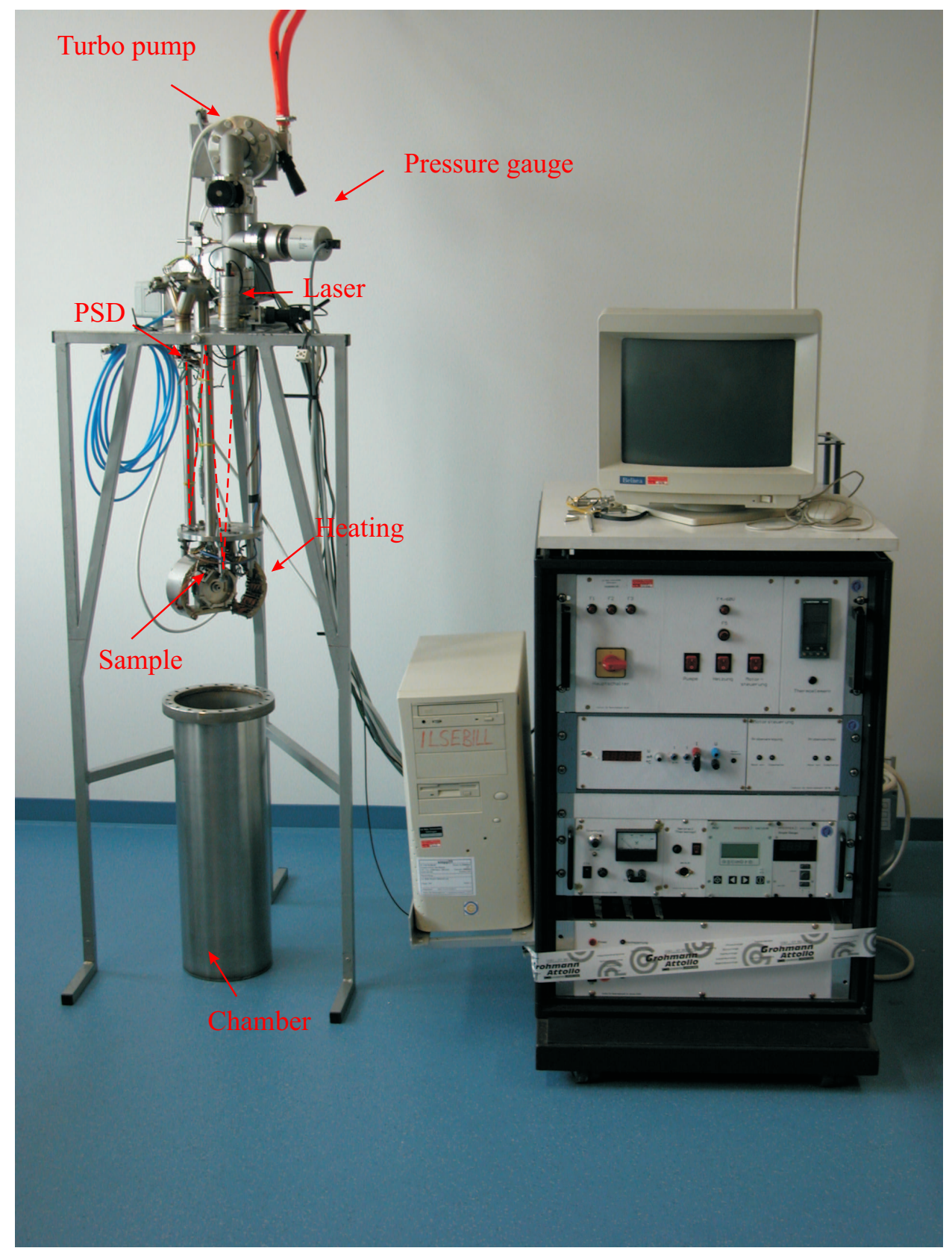

Figure 4.1: The VRA with the opened vacuum chamber. The dashed line is drawn where the laser beam passes. 


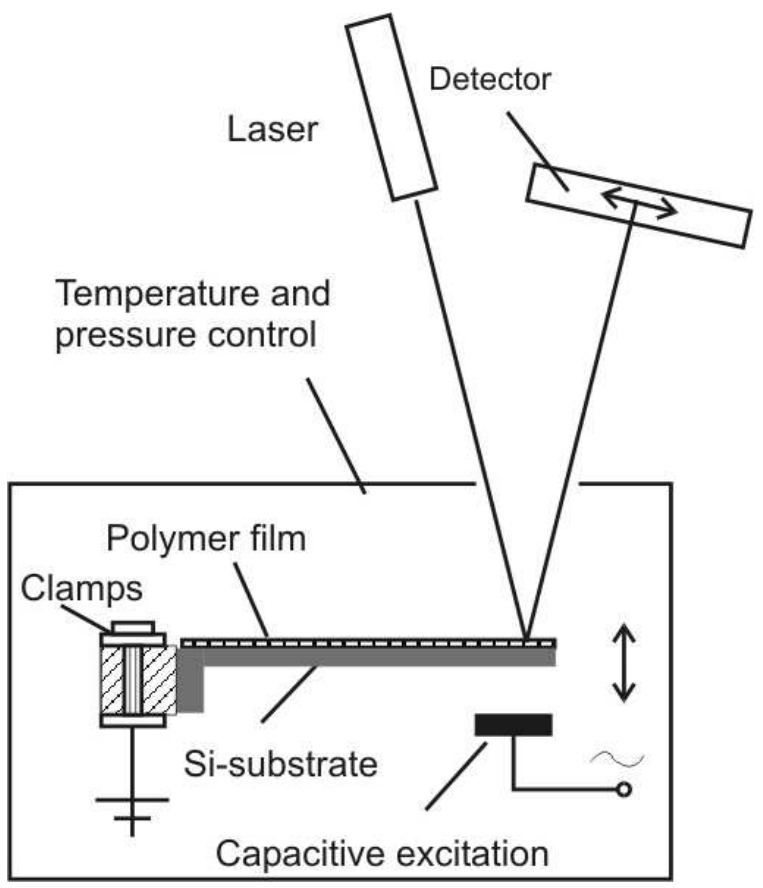

Figure 4.2: The simplified scheme of the VRA.

polymers is that of Neuhauser et al. (2004), where the mechanical properties of unstable thin poly(1,4-phenylen-vinylene) films are studied by means of the vibrating reed method.

In this work supported polymer films of thicknesses from $8 \mathrm{~nm}$ up to several microns were studied. The advantage of the method compared to the dielectric spectroscopy is that one surface of a film studied remains free of contact with a different material. This free surface plays a key role in the analysis of the influence of reduced dimensions on glass transition (see Section 5.1.1). The method does no give only the information about the $T_{g}$, but also about the whole relaxation spectrum.

A polymer film is applied on a Si substrate, mostly by spin-coating (for films of several microns a polymer solution is applied on the substrate without spinning). One side of the sample is wrapped in $\mathrm{Al}$ foil and clamped between two copper plates of the sample holder. The sample is placed in the vacuum chamber and a damping of free vibrations is measured at the temperature range appropriate for the material. The sample is capacitively excited at its free end to the flexural bending. A laser beam is reflected from the top of the sample to the position sensitive detector (PSD). The free decay of the displacement amplitude resulting from the internal friction is measured as the position of the reflected laser beam on the detector in time. The heating rate is $0.2 \mathrm{~K} / \mathrm{min}$ and it is considered constant for one decay measured (see Fig 4.14). The actual temperature is measured by a 
Pt1000 resistance device which is in contact with the copper plates of the clamps. The film thickness is calculated from the shift in the eigenfrequency of the sample (Eq. 3.20) and proved by microscopic methods described in 4.3 .

A photo of the apparatus is given in Fig. 4.1 and the focused sample holder with a sample mounted in Fig. 4.13. The simplified scheme of the apparatus is depicted in Fig. 4.2.

Different effects in the material cause the internal friction. In the experiment, not only the material-specific damping is measured, but also some other artifacts (some of the artifacts are described in Section 3.2) which have to be subtracted.

\subsubsection{Silicon cantilevers and clamps}

All the cantilevers in the VRA experiments are of a rectangular form. The advantage of the shape is in the possibility to make rather accurate and clear calculations as shown in Chapter 3. However, for spin-coating of uniform films, an ideal shape would be circular.

One of the key technical problems is to reduce the damping of the substrate, in order to observe relaxation processes in thin polymer films. For measurements based on mechanical damping, the substrates are often made of $\mathrm{Si}(100)$ single crystal, due to its low damping. Si is anisotropic and the damping of $\mathrm{Si}(100)$ is for about $25 \%$ lower then that of $\mathrm{Si}(110)$. The $\mathrm{Si}(100)$ wafers of which the substrates are made of are slightly Phosporus or Boron doped in order to increase the conductivity, necessary for the capacitive excitation of vibrations.

The dominant damping mechanism in Si reeds is the thermal damping $Q_{t h}^{-1}$ and since in the case of a rectangular reed $Q_{t h}^{-1} \propto d_{s}^{3} / L^{2}$ (Eq. 3.38), the thinner and longer the reed is the lower the thermal damping will be. $\mathrm{Si}$ single crystal wafers are very brittle; therefore the limits of the dimensions are set by production, film preparation and by mounting the sample in the apparatus. Due to their different natural frequencies, impurities and complexity of production, with one Si-reed as a substrate one has to be capable to perform measurements with several films. Polymer films if not cross-linked may be dissolved in appropriate solvents from the substrates. The covalent bonds established by cross-linking can be destroyed by heating the sample in a furnace at $500^{\circ} \mathrm{C}$. At this temperature both PS and PVCN disintegrate, but one has to avoid oxidizing of Si. A thick oxide-layer can influence the modulus and the frequency of the substrate, which can affect the calculation of the film thickness.

It is very important how the sample is clamped. The oscillation mode exhibits an inhomogeneous stress distribution in the clamping area and, more problematic, considerable friction at the clamps (Weller, 2001). The friction between the reed and the clamps may cause an energy loss that is 


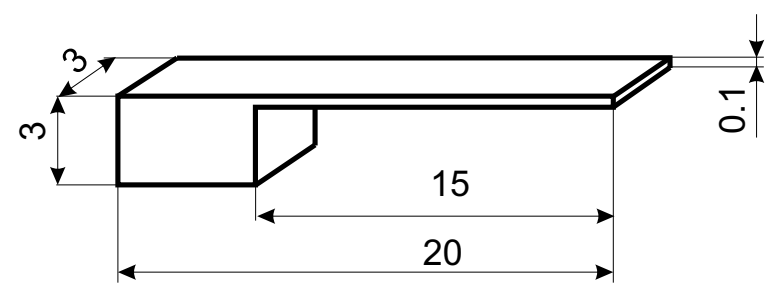

Figure 4.3: $\mathrm{Si}(100)$ wafers are cut to $3 \times 3 \times 21 \mathrm{~mm}^{3}$ strips and polished so that the thick part remains for clamping and the thin reed exhibits low damping.

even higher than the thermal damping. It is important that the support is rigid and massive in relation to the specimen. For that reason, the substrates are made in that way that the clamped end of a specimen is rigid. The clamps have to be made of a material that is a good heat conductor, but also does not diffuse fast into $\mathrm{Si}$ at experimental temperatures.

In the next paragraphs, the reeds and the clamps used in this work are described. Additionally, a brief description of one reed that was not acceptable is given, pointing out its disadvantages.

\section{Polished cantilevers}

These substrates are produced at the Institute of Materials Science, University of Tsukuba, Japan. Strips of a silicon single crystal of $3 \times 3 \times 21 \mathrm{~mm}^{3}$ are cut out of $\mathrm{Si}(100)$ single crystals (B-doped p-type) and polished to thin reeds with one end left thick for clamping (see Fig. 4.3). In that way, by vibrating, only the thin part moves. Thus the friction between the reed and the clamps is reduced. The function of the thick part is also to make the handling easier. The top surface of the reeds is a mirror surface (Fig. 4.4A) and the back surface is smooth, but slightly undulated by $\pm 5 \mu \mathrm{m}$ along the long axis. The edges of the reed are rather rough. The gauge length of the reed is $15 \mathrm{~mm}$. The eigenfrequency of the substrates at $300 \mathrm{~K}$ are between $598 \mathrm{~Hz}$ and $640.5 \mathrm{~Hz}$, depending on the reed. The thickness of the reeds calculated from their natural frequencies (Eq. 3.18) is about $105 \mu \mathrm{m}$, what is in agreement with the microscopic pictures (Fig. 4.4). The relaxation time is $1.24 \cdot 10^{-5} \mathrm{~s}$ (Eq. 3.37) and the thermal damping $Q_{t h}^{-1}=10^{-5}(\mathrm{Eq}$. 3.36).

The clamps for this kind of substrates are made of two copper plates, jointed by two screws as depicted in Fig. 4.5. In order to prevent a very fast diffusion of $\mathrm{Cu}$ atoms into Si crystal (Runyan, 1965), the thick part of the reed is first wrapped in $\mathrm{Al}$ foil and then built between the two copper plates. The reed has to be placed in the middle of the clamps. The screws are tightened till the upper plate bends.

The measured $Q^{-1}$ factor of these substrates is about $1.5 \cdot 10^{-5}$ at $300 \mathrm{~K}$. Therefore, losses that were not caused by the thermal damping as calculated 

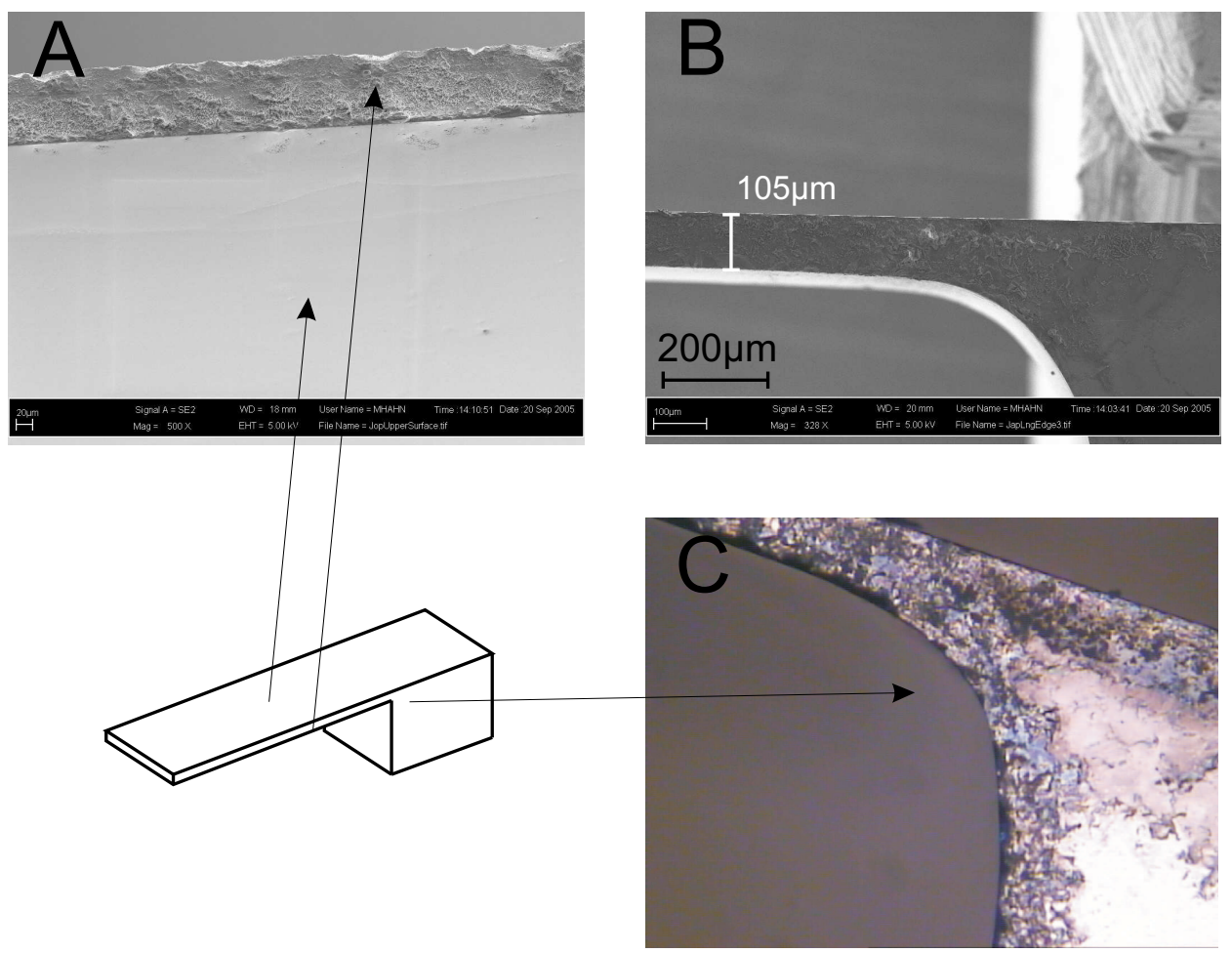

Figure 4.4: A and B are SEM pictures of a polished reed. The reed under the optical microscope can be seen in $\mathrm{C}$.

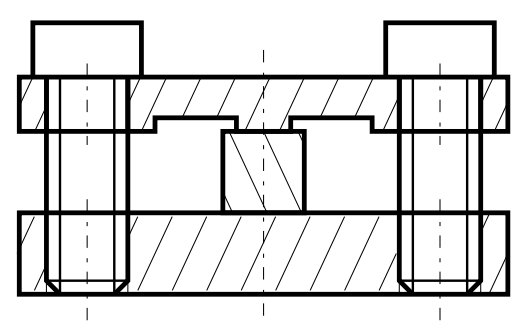

Figure 4.5: The cross-section of the thick part of the Si substrate (in the middle), clamped between two copper plates. 

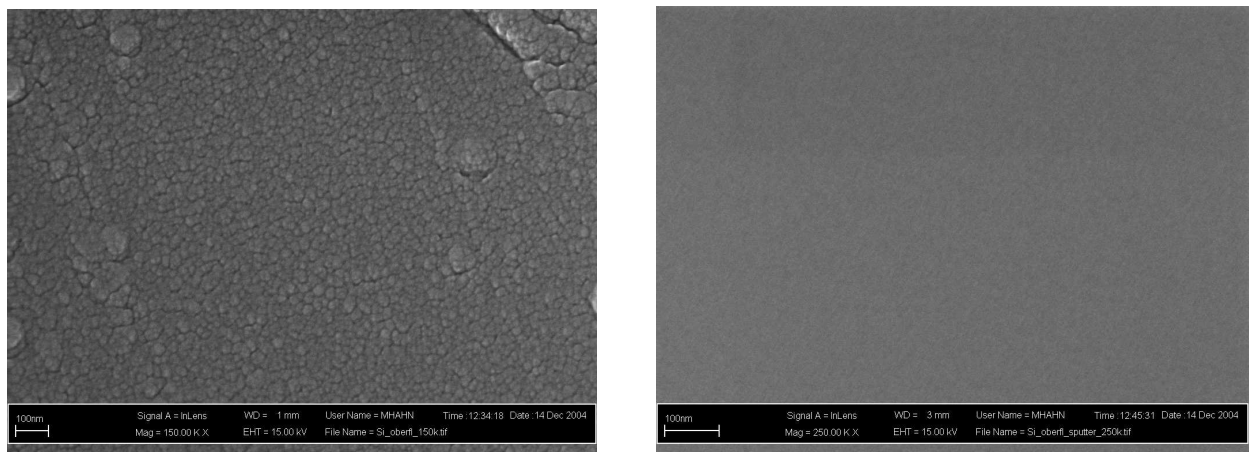

Figure 4.6: SEM pictures of the lower surface of the polished reed before (1.) and after (r.) dry etching.

from Eq. 3.36 are about $0.5 \cdot 10^{-5}$.

\section{Dry etched cantilever}

The $Q^{-1}$ factor of the above described substrates is too high for observing the $\alpha$ relaxation in films of thicknesses below $100 \mathrm{~nm}$. The thermal damping $Q^{-1}$ can be reduced by making the reed thinner (Eq. 3.38). The lower surface of a polished sample received from Japan is therefore sputtered by Ar ions under the angle of $90^{\circ}$ in order to have a uniform thickness. After sputtering, the surface became smoother (see Fig. 4.6).

The eigenfrequency of the reed at $300 \mathrm{~K}$ after sputtering is $238 \mathrm{~Hz}$, what corresponds to a thickness of $39 \mu \mathrm{m}$. The relaxation time of a reed of that thickness is $1.7 \cdot 10^{-6} \mathrm{~s}$, what infers $Q_{t h}^{-1}=1.35 \cdot 10^{-6}$. The measured $Q^{-1}$ factor was $6.5 \cdot 10^{-6}$.

Here one can observe that the difference between the calculated thermal loss and the measured loss is the same as by the sample before sputtering, 5 . $10^{-6}$. The shape of the reed and the clamps are the same in both cases; hence these additional losses can be attributed to friction with clamps, acoustic noises, friction with the air left after evacuating and the other losses in the experimental setup.

Usually, in mechanical spectroscopy, the inconvenience for measurements with different frequencies is considered as a big disadvantage. By dry etching two frequencies are achieved. The difference in the eigenfrequency is used for the calculation of the activation energy of the $\alpha$ relaxation (Chapter 5).

The EDX analysis showed that the sample is contaminated by atoms from the steel sample holder needed for sputtering (see Appendix). Since the properties of the reed were not known any more due to the contamination and the eigenfrequency and the modulus are important for ultra-thin films the sample was not appropriate for the measurement concerning reduced dimensions. Further chemical treatment of the sample in order to remove 

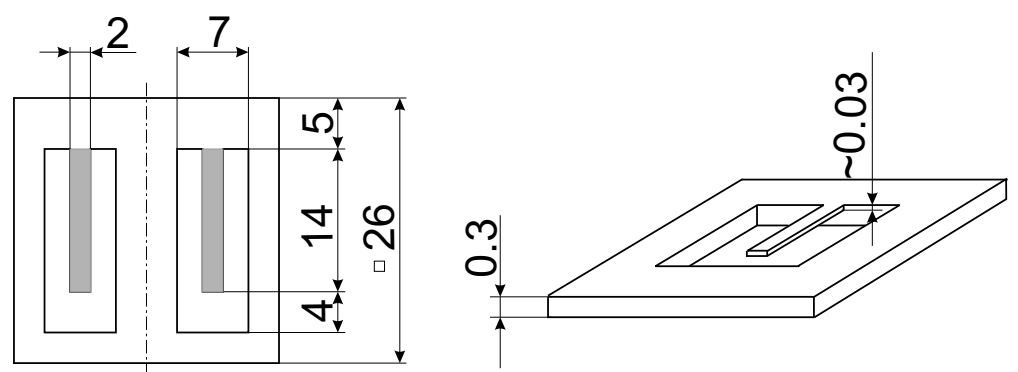

Figure 4.7: On the left hand side is the top view of the wet etched cantilever after etching. The dimensions $26 \times 26 \mathrm{~mm}^{2}$ are determined by the size of the etching mask. The gray colored surfaces are the vibrating reeds. After etching the break is done on the dash-dotted line. The sketch is proportional to the real dimensions. On the right hand side is the $3 \mathrm{D}$ projection of the substrate. The sketch is not proportional to the real dimensions.
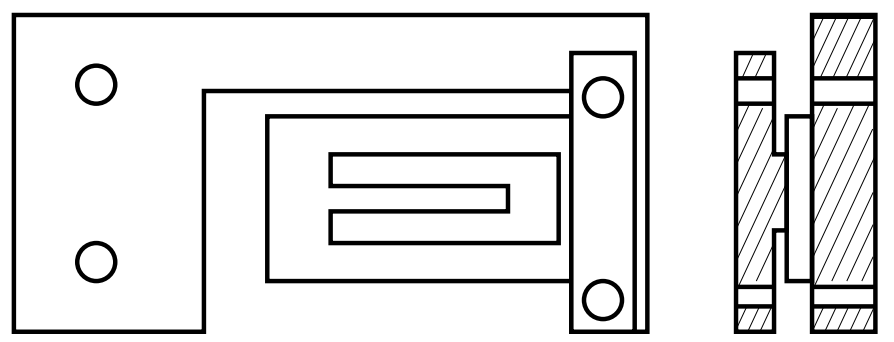

Figure 4.8: The frame of the cantilever is clamped on the side which is not in contact with the vibrating reed. This is done in order to avoid friction in clamps. The contact surface of the upper $\mathrm{Cu}$-plate is reduced in order to avoid breaking the frame. The plate slightly bends by screwing. The holes on the left hand side are needed for mounting the sample holder in the apparatus.

the unwanted foreign atoms would ruin the mirror surface which is necessary both for the spin coating of thin films as for reflecting the laser beam from the surface onto the detector for measuring the displacement of the reed.

\section{Wet etched cantilevers}

The wet etched substrates are a good solution for reducing the damping, necessary for observing dynamical behavior of thin polymer films. The samples are produced in cooperation with the Institute of Semiconductor Technology in Braunschweig by anisotropic etching in potassium hydroxide $(\mathrm{KOH})$. The wafers from which the resonators are etched were $300 \mu \mathrm{m} \mathrm{Si}(100)$ P-doped n-type. The maximal surface of the etching mask is $26 \times 26 \mathrm{~mm}^{2}$; therefore two samples consisting of a frame $13 \times 26 \times 0.3 \mathrm{~mm}^{3}$ each and a reed of about $30 \mu \mathrm{m}$ thickness are etched at the same time (see Fig. 4.7). After etching the frame was broken in the middle, in Fig. 4.7 marked as a dash-dotted line. When excited to a vibration at the resonant frequency of the thin reed 

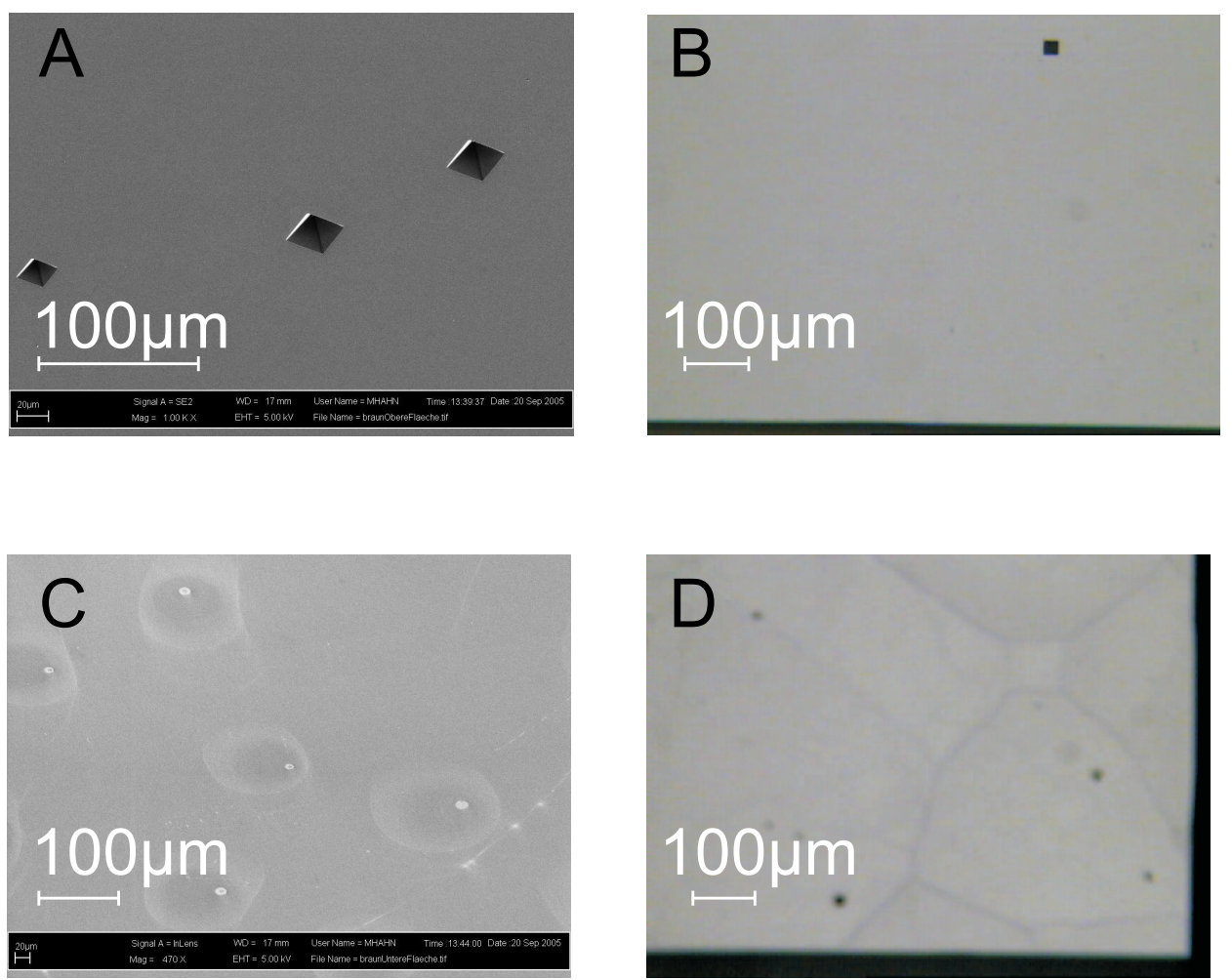

Figure 4.9: The wet etched reed observed by SEM (A and C) and optical microscope (B and D). A and B are the upper surfaces, $\mathrm{C}$ and $\mathrm{D}$ are the lower surfaces. The upper surface contains a few pyramid imprints resulting from the damages in the etching mask. The pyramidal shape is caused by the anisotropy of Si. The lower surface looks like the orange peel. The surface can be nicely seen under the light microscope in Fig. D. 


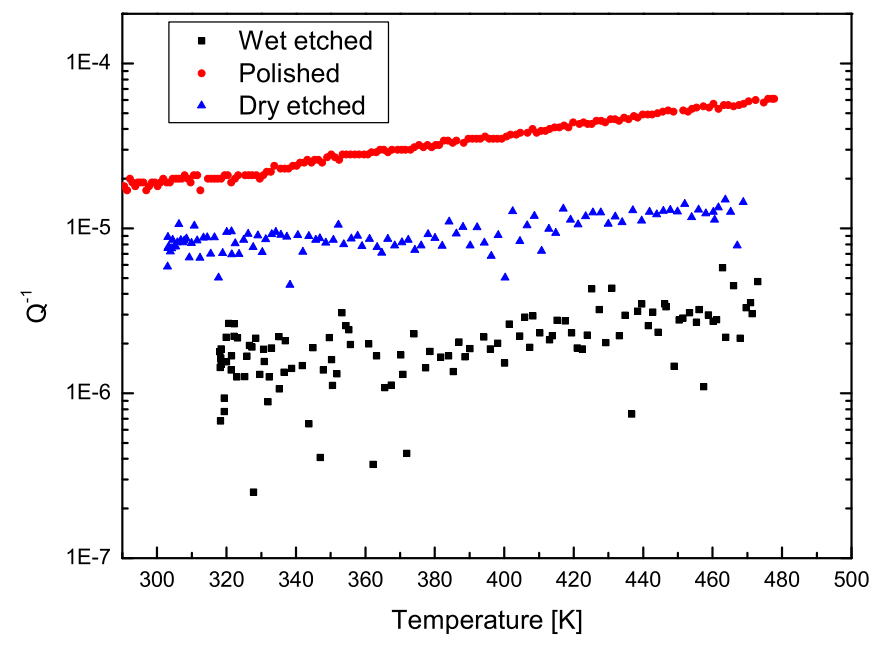

Figure 4.10: Damping of different substrates. With the wet etched substrates the detection limits of the VRA are reached.

the frame does not vibrate and the loss in clamps is reduced. The frame also serves as a protection of the thin reed from breaking, by clamping or spin-coating.

In order to clamp the sample as far from the reed as possible, the sample holder was designed as depicted in Fig. 4.8. The material of which the sample holder was made is copper and again between the Si surface and the copper plate a thin $\mathrm{Al}$ foil was put in order to prevent diffusion of $\mathrm{Cu}$ into Si.

The eigenfrequency of these samples is between 100 and $220 \mathrm{~Hz}$ corresponding a thickness of 17 to $36 \mu \mathrm{m}$. The relaxation time of the thermal relaxation is ranging from $3.2 \cdot 10^{-7}$ up to $1.5 \cdot 10^{-6}$ and the $Q_{t h}^{-1}$ resulting from the thermal damping is between $10^{-7}$ and $3 \cdot 10^{-7}$.

The measured $Q^{-1}$ is about $10^{-6}$. It seems that this is the detection limit of the method.

The microscopic analysis of the wet etched reed shows that the upper surface on which films are spin-coated is very smooth with rare pyramid imprints following the crystal planes. The imprints are damages caused by some defects in the etching mask. On the lower surface the etching causes the appearance of orange peel, which does not play an important role since polymer films are not in contact with it.

The spectra of the substrates described above are given in Fig. 4.10. 


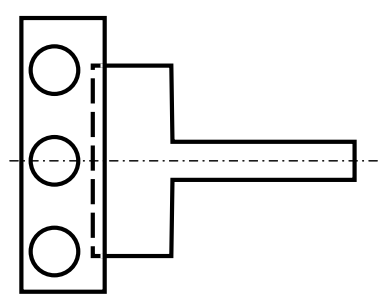

Figure 4.11: The laser cut cantilever and the respecting sample holder. The size of the vibrating reed is the same as that of the polished sample.
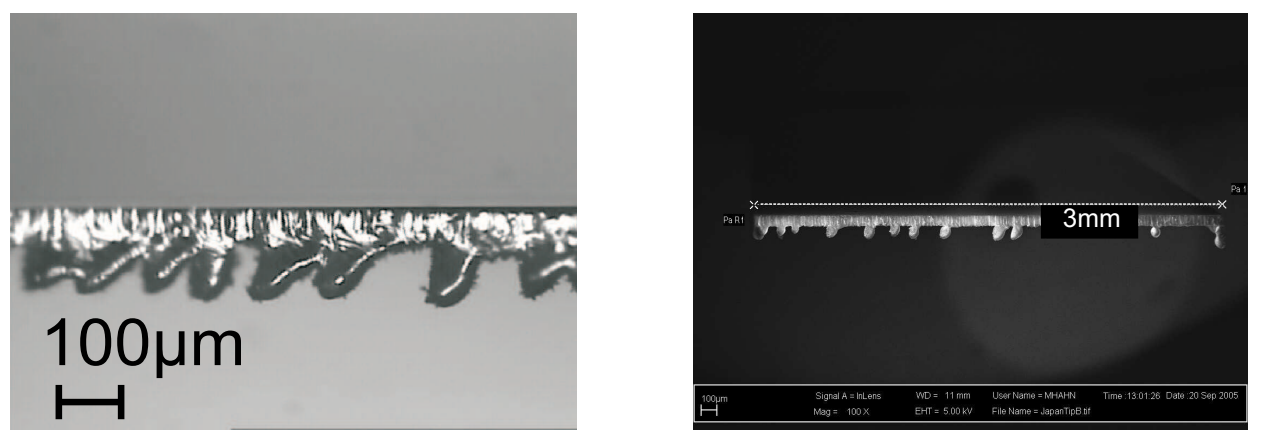

Figure 4.12: Droplets at the vibrating reedresulting from the laser cut. On the left hand side the picture was obtained by the optical microscope, on the right hand side by SEM.

\section{A laser cut cantilever}

This sample is cut by a laser from a $60 \mu \mathrm{m} \operatorname{Si}(100)$ single crystal wafer at the Fraunhofer Institute for Laser Technique in Aachen. It was assumed that cutting a $\mathrm{T}$ form out of the wafer and clamping the substrate a few millimeters away from the vibrating part as depicted in Fig. 4.11 will be enough to reduce the damping in clamps. However, the $Q^{-1}$ factor measured for these samples is $6 \cdot 10^{-3}$, although the length was the same as for polished reeds described above and the thickness was $40 \mu \mathrm{m}$ lower. The microscopic pictures of the reed can be seen in Fig. 4.12. The laser cutting leaves droplets on the edges of the substrate. Therefore the samples were not used.

\subsubsection{Excitation}

As depicted in Fig. 4.2 and in Fig. 4.13 a sample is excited capacitively. A slightly doped Si reed makes a parallel plate type capacitor with a brass screw connected to the alternating current. The frequency can be changed by a frequency generator. The excitation takes place when the excitation frequency is close to the resonant frequency of the reed. The screw is placed 
below the tip of a reed; therefore only the first vibration mode can be excited.

The electrostatic force, $\mathrm{F}$, between the parallel plates is

$$
F=\frac{1}{2} \frac{\epsilon_{0} A}{d^{2}} U^{2},
$$

where $\epsilon_{0}$ is the permittivity of free space $\left(\epsilon_{0} \approx 8.854 \cdot 10^{-12} \mathrm{~F} / \mathrm{m}\right), A$ is the surface of the plates, $d$ is the distance between the plates and $U$ is the voltage.

If only an $\mathrm{AC}$ voltage

$$
U=U_{0} \cos \left(\omega_{R} t\right)
$$

with an amplitude $U_{0}$ and the resonant frequency $\omega_{R}$, is applied, the electrostatic force will be

$$
F=\frac{1}{4} \frac{\epsilon_{0} A}{d^{2}} U_{0}^{2} \cos \left(1+2 \omega_{R} t\right) .
$$

This means that the force oscillates with the frequency that is twice as big as that of the AC voltage. With $A=3 \cdot 10^{-6} \mathrm{~m}^{2}, d=10^{-3} \mathrm{~m}$ and $U_{0}=10 \mathrm{~V}$, the approximate value of the amplitude force would be $F=6 \cdot 10^{-10} \mathrm{~N}$. In order to increase the force between the two plates a bias-direct voltage $U_{\text {bias }}=180 \mathrm{~V}$ is applied. The overall voltage in this case is

$$
U_{\text {sum }}=U_{\text {bias }}+U_{0} \cos \left(\omega_{R} t\right) .
$$

Inserting Eq.. 4.4 into Eq. 4.1, the force will be

$$
F=\frac{1}{2} \frac{\epsilon_{0} A}{d^{2}}\left[U_{\text {bias }}^{2}+2 U_{\text {bias }} U_{0} \cos \left(\omega_{R} t\right)+\left(U_{0} \cos \left(\omega_{R} t\right)\right)^{2}\right] .
$$

$U_{\text {bias }}$ is a direct voltage and has no influence on the resonant frequency. $U_{0}$ is between 1 and $15 \mathrm{~V}$, depending on doping, film thickness, distance $d$, therefore $2 U_{\text {bias }} U_{0}>>U_{0}^{2}$. This yields the force proportional to $U_{\text {bias }} U_{0}$

$$
F \approx \frac{\epsilon_{0} A}{d^{2}} U_{b i a s} U_{0} \cos \left(\omega_{R} t\right) .
$$

Adding the bias, with $U_{0}=10 \mathrm{~V}$, the force increases 72 times, giving $4 \cdot 10^{-8} \mathrm{~N}$. In this case is the AC-frequency the same as that of the force of the first mode frequency.

The amplitude of the reed can be adjusted by changing the AC voltage or the distance of the plates. With the $3 \mathrm{~cm}$ long PSD, the maximal amplitude of the tip of the reed, $a$, that can be read may be calculated from the geometry of the apparatus as $a / L_{s u b} \approx 0.5 \cdot L_{P S D} / L_{\text {beam }}$. The maximal vibration angle is $\tan ^{-1}(1.5 / 100)=0.86^{\circ}$ (see Subsection 4.1.3) and for the substrate length $\mathrm{L}=15 \mathrm{~mm}$ the maximal amplitude is $225 \mu \mathrm{m}$. For all 


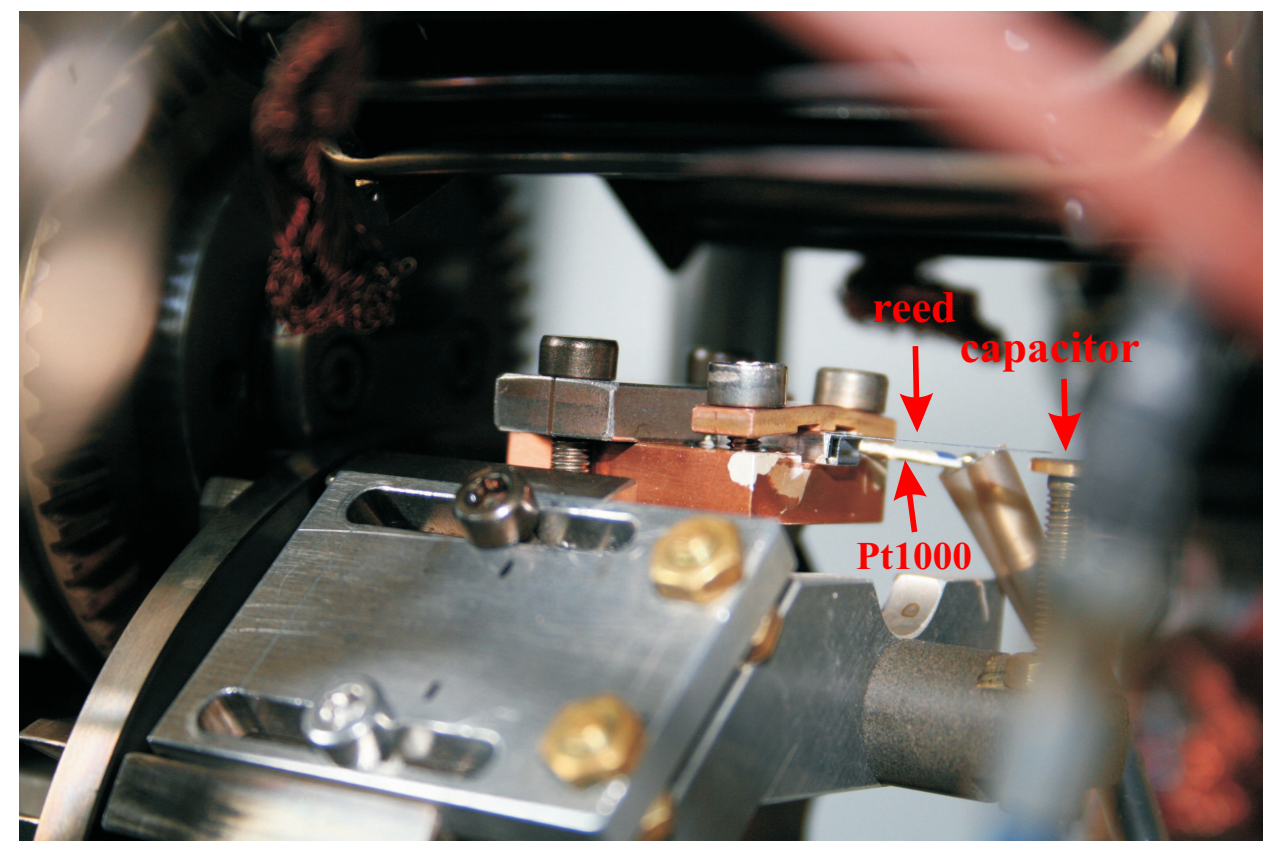

Figure 4.13: A detail of the VRA, the reed clamped in the sample holder, a parallel plate capacitor for the excitation, the Pt1000 inserted between the two copper plates of the sample holder and connected with conductive silver.

substrates the damping is independent of the amplitude. This can be seen in Fig. 4.14. The decay is exponential; hence the result is independent of the moment or the amplitude when the measurement (or calculation of the exponent) starts. During the full temperature scan of $200 \mathrm{~K}$ with 0.2 $\mathrm{K} / \mathrm{min}$ (16 to 17 hours), both the resonant frequency of the sample and the amplitude are influenced by changes of the elastic modulus; therefore both are tested for each damping measurement.

\subsubsection{The laser and the position sensitive detector (PSD)}

A Si based PSD is an optoelectronic device which uses the lateral photovoltaic effect to determine the position of a light spot on a semiconductor surface. A laser beam used as the light source allows the object studied to be positioned relatively far away from the PSD. In this case the sensitivity of the laser-PSD system increases significantly and is independent of the size of the laser point.

A red laser beam of class 2 with a wavelength of $670 \mathrm{~nm}$ and the maximal power output less then $1 \mathrm{~mW}$ passes through a small pinhole and hits the mirror surface of the substrate close to the top. The beam is reflected from the substrate surface to the PSD. In order to increase the path of the reflected beam a system with two adjustable mirrors is designed increasing 

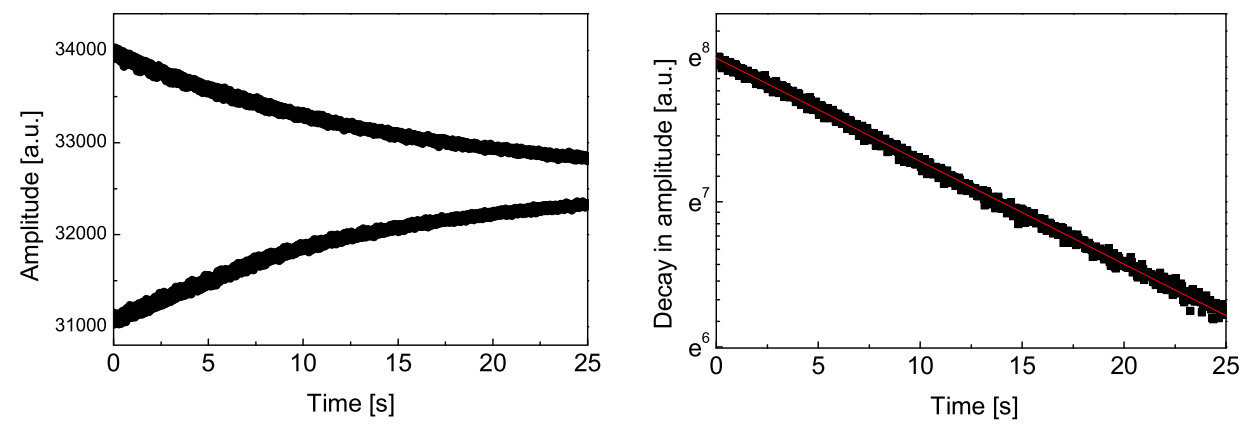

Figure 4.14: A decay in amplitude of a vibrating reed. Here, on the left hand side, only the extremes, minima and maxima, are depicted, whereas on the right hand side there is the difference between them in a log scale.

the length of the incident beam to about $40 \mathrm{~cm}$ and of that the reflected beam to about $100 \mathrm{~cm}$. The length of the one dimensional PSD used in this experiment is $3 \mathrm{~cm}$.

The difference in the photocurrent on both edges of the detector is converted into voltage by means of the PSD-electronics, which is then transfered by the A/D converter to the PC.

The A/D converter is a $\mathrm{PC}$ card programmed by a $\mathrm{SW}$ to convert linearly the voltage between -10 and $10 \mathrm{~V}$ into 16 bit data. The maximal sample rate for one channel is $100 \mathrm{kHz}$.

\subsubsection{Controlling and measuring the temperature}

The temperature control is a very important feature when the behavior of polymers is observed. The increase in temperature, if too fast, may influence the effect of the reduced dimensions on $\alpha$ relaxation.

As it can be seen in Fig. 4.1, there is a heating system made of two wound heating resistors around the sample holder and the sample. Moreover, the heater and the sample holder are connected by several copper wires to increase the heat flow. In this way the heat is transfered both by radiation and by conduction.

The temperature is controlled by a PID regulator. The actual temperature is read by a thermocouple of a type $\mathrm{K}$ which is placed close to the sample. The measurements are done with the heating rate of $0.2 \mathrm{~K} / \mathrm{min}$.

Additionally, a Pt1000 sensor is placed between the copper plates of the sample holder very close to the vibrating reed. The conductivity is improved by conductive silver. The temperature measured in this way is taken as the actual value presented in the results. 


\subsubsection{Vacuum system}

A vacuum system is required to avoid losses caused by friction of the vibrating reed with gas molecules (Eq. 3.39) and to prevent oxidizing of polymer films. A vacuum in the chamber of the order of magnitude $10^{-7}$ to $10^{-6}$ mbar is reached by a turbomolecular pump backed by a diaphragm vacuum pump.

\subsubsection{Precision of the VRA}

The resolution of the PSD given from the producer for optimal conditions is up to $2 \mu \mathrm{m}$. It is independent of the size of the laser. However, a small error can be caused due to the dispersion of the light after the transmittance through the polymer films. The error appears, because the area affected by the leaser beam changes due to the vibrations. The maximal offset angle by vibrations is $0.86^{\circ}$ and the surface of the affected area affected by the laser beam is up to $4 \mathrm{~mm}^{2}$, hence the increase in the area is about $0.01 \%$. The PC card with the sample rate of $100 \mathrm{kHz}$ assures the determination of a maximum of a $2 \mathrm{kHz}$ sine signal to the accuracy of $2 \%$.

The largest error of the system results from the noise in vibrations due to the acoustic excitations, pumps, stability of the apparatus etc.

The difference in the temperature read compared to the real temperature of the substrate is about $2 \mathrm{~K}$, but since the temperature is measured always at the same position, the deviation is constant for all experiments. This allows a correct measuring of the shift in the position of the relaxation peak. In the experiments described in Chapters 5 and 6 the effect observed is the changes in the relaxation peak; therefore the exact temperature does not have a high relevancy for the experiment.

\section{$4.2 \quad$ Spin-coating of films}

Thin polymer films are produced by applying a dilute solution of a polymer in a volatile solvent uniformly on a substrate which then rotates. The film surface and thickness may be influenced by solution concentration, spinning velocity, evaporation constant and the substrate shape. The solutions made out of a nonvolatile solvent on a circular substrate have the best thickness uniformity. The convective radial flow of the solvent governs film thickness during the early stages of the process, while the solvent evaporation becomes significant in later stages. A good description of the process and the influencing parameters is given in (Birnie III and Manley, 1997).

For the preparation of polymer films analyzed in this work, the spin coater P-6708D produced by Cookson Electronics is used. The speed can be varied between 100 and 8000 RPM. The parameters chosen for spin-coating 


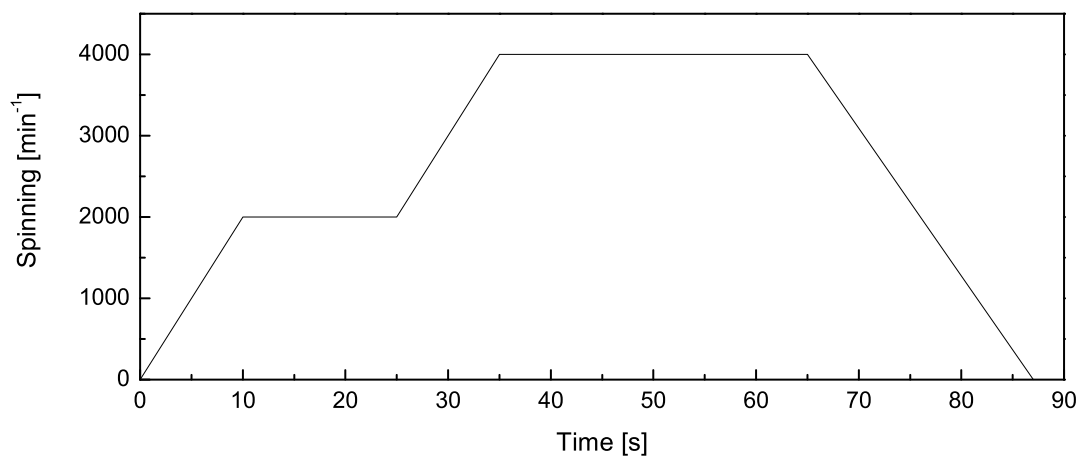

Figure 4.15: The spin-coating cycle.

the thin films are shown in Fig. 4.15. The thickness is varied by changing the solution concentration. Small variations in the initial dispense volume yield the same spinning behavior resulting in the same film thickness (Birnie III and Manley, 1997).

The film characterization and the measurements of the film thickness by the dual FIB/SEM system is performed on substrates prepared only for the microscopy to avoid handling with the very sensitive and complex vibrating reeds. Since the spin-coated films are dependent on the geometry of the substrate, the measured thicknesses are not the same as those of the films spin-coated on cantilevers, but are close to them.

Before any analysis is done the sample has to be annealed at a temperature above $T_{g}$ for several hours for two reasons: (i) The "skin" formation resulting from the enrichment of solute component on the surface by evaporation inhibits further evaporation of the remaining solvent. Heating above $T_{g}$ makes the evaporation faster. (ii) The second reason is to relax stresses in the film caused by spinning and evaporating the solvent.

The substrate is held by a vacuum chuck. The upper surface of the substrate must be polished in order to get a film as uniform as possible and the lower one may be slightly undulated, but still it must be appropriate for the vacuum holding.

PS is dissolved in toluene and PVCN in tetrahydrofuran, both volatile. More information on solubility of polymers in different solutions can be found in Van Krevelen (1990). 


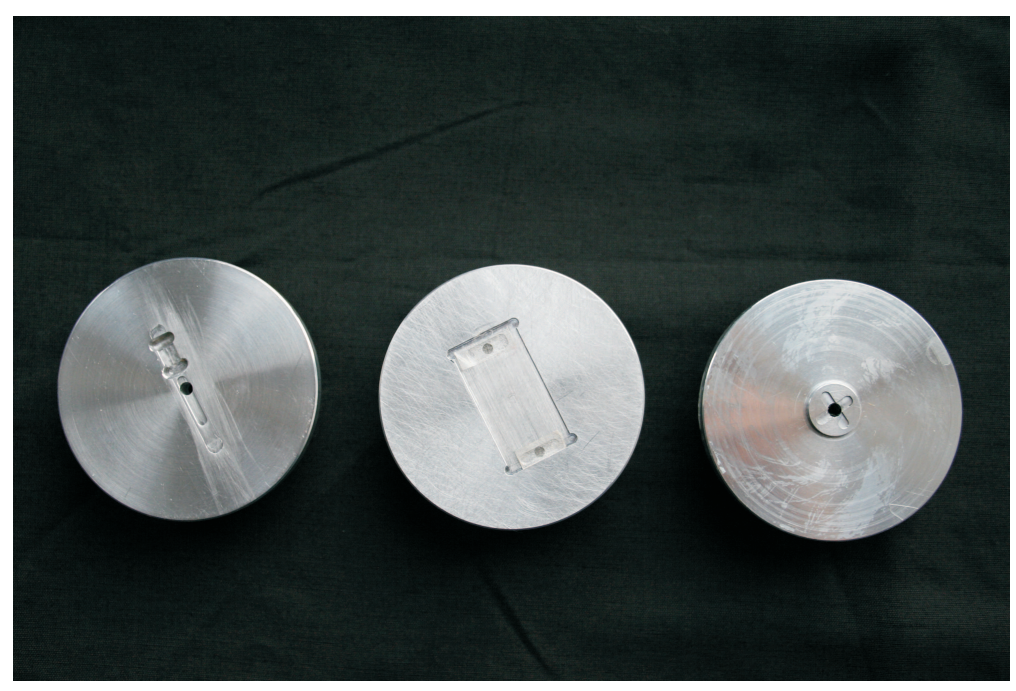

Figure 4.16: The three different sample holder for spin-coating. The first one from the left is appropriate for the polished and the dry etched reed, the second one is for the wet etched reed and the third one is appropriate for simple squared substrates used for FT-IR and FIB/SEM analysis.

\subsubsection{Vacuum chucks}

There are three different sample holders used in these experiments as presented in Fig. 4.16. Each holder is meant for the sample of a different shape. In order to spin-coat the surface as uniform as possible a substrate has to be placed in the middle of the sample holder, on the rotational axis and its surface must be perpendicular to the axis. The tight clearance between the exchangeable sample holder and the spin-coater, needed for the vacuum, is achieved by using a Teflon ring. If possible, the sample holder should have the holes drilled so, that the frame of the substrate is held and not the thin reed itself, in order to avoid bending of the substrate due to the vacuum holder and sticking of the reed to the sample holder due to the unwanted flow of the solution sucked between the reed and the sample holder.

\subsection{Focused Ion Beam (FIB) and Scanning Elec- tron Microscopy (SEM)}

The thickness of films analyzed by mechanical spectroscopy was calculated from the difference in the eigenfrequency of the pure substrate and the composite substrate-film (Eq. 3.20). In order to prove whether the calculation gives the exact values even for very thin films, a precise cross-sectioning analysis with the FIB/SEM dual platform instrument is performed. The electron beam is used to view the cross-section face as the ion beam mills 
normal to the sample surface. The sample is prepared by spin-coating the same solution as that used for the films applied on the vibrating reed, with the same spinning parameters, on a Si substrate of $1 \mathrm{~cm}^{2}$. Slight differences are to be expected due to the different geometry of the substrate. The instrument used is produced by FEI, type Nova 600 NanoLab DualBeam (FIB/SEM). The liquid metal ion source (LMIS) is gallium. An electric field of $10^{8} \mathrm{~V} / \mathrm{cm}$ is applied on a tungsten needle, the tip of which is wetted by Ga. The extraction voltage pulls Ga from the $\mathrm{W}$ tip and ionizes it by field evaporation of the metal. The instrument has a gas source, needed for the chemical vapor deposition of Pt.

A small film surface of about $2 \times 1 \mu \mathrm{m}^{2}$ is covered by a Pt layer on the film surface first deposited by the electron beam (low energy deposition prevents the damaging of the polymer layer) and then the deposition is continued by ion beam (faster) (see Fig. 5.18). The layer has two roles, one is to protect the film and the other one is to make an interface with the film, which makes the thickness measurement easier. The cross-section of the layered sample is milled by FIB. The milling is achieved by accelerating gallium ions which etch off the exposed material, leaving a very clean hole. The imaging of the cross-section is done by the SEM. A good introduction into FIB and FIB/SEM dual platform systems can be found in (Giannuzzi and Stevie, 2005) and some more detailed physics about the topic can be found in (Orloff et al., 2005)

The SEM analysis of the substrates described in this Chapter is done by LEO SUPRA ${ }^{\mathrm{TM}} 35$ with a resolution between 1.3 and $2.1 \mathrm{~nm}$ at the acceleration voltage of $5 \mathrm{kV}$.

The SEM imaging of the cross-linked thick polymer film described in Section 6.4 is done by Philips SEM 515 with the LaB6 cathode and the resolution of $5 \mathrm{~nm}$. The analysis was done with $25 \mathrm{kV}$. 


\section{Chapter 5}

\section{Effect of Reduced Dimensions on the Glass Transition of Thin Polystyrene Films}

\subsection{Introduction}

The study and application of very small systems are driven by nanotechnology and by need and desire in science to understand the physical properties of materials whose sample size is in the nanometer regime. It is still not completely understood how the properties of the small systems might differ from those of bulk systems. Different effects such as the large surface area to volume ratio for instance may influence the dynamics of molecules near the glass transition and hence the properties of the material. In particular, studies of the glass transition and dynamics in thin polymer films offer great promise to increase the understanding of dynamics in polymeric systems and in glass forming systems in general. The nature of the glass transition is an important and unsolved problem and a proper explanation of the thin film data may provide an elucidation of fundamental physics behind the glass transition (Sharp and Forrest, 2003).

In recent years many studies on the glass transition at the nanometer length scale have been conducted, but often with conflicting results and even fundamental questions regarding the behavior of polymer chains near surfaces are poorly understood. An increase, decrease or no effect of reduced dimensions on molecular mobility have been reported depending on the experimental method.

The experimental study of size effects on the glass transition consists of two major types of measurements: thermodynamic-type measurements of the glass transition and dynamic-type measurements of the $\alpha$ relaxation 


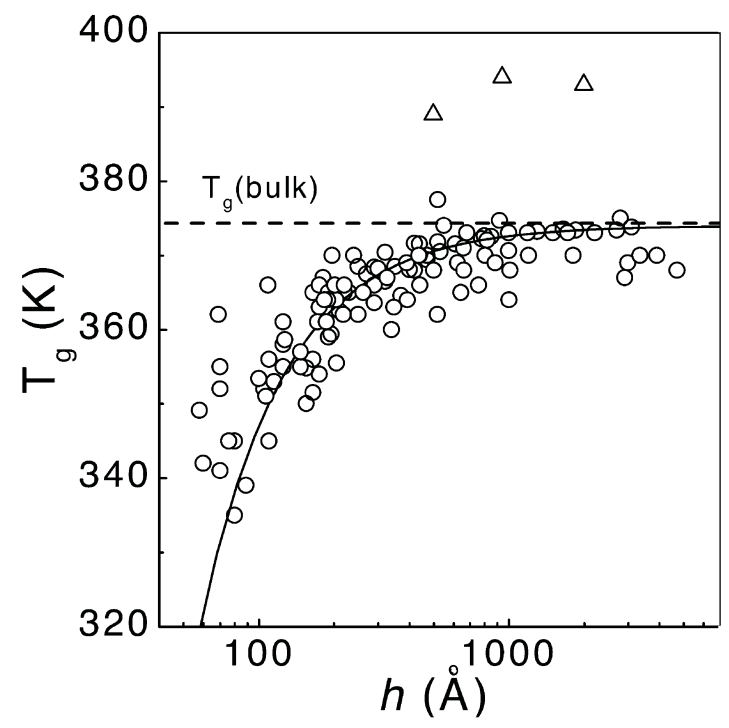

Figure 5.1: Measured values for the glass transition temperature $T_{g}$ of thin supported PS films. The data was reported using different techniques and substrates. (Forrest and Dalnoki-Veress, 2001)

associated with $T_{g}$ or molecular mobility. The thermodynamic measurement is defined as a direct measurement of a thermodynamic property such as heat capacity or volume versus temperature, where a break or jump in the thermodynamic property is seen at the transition temperature (see Fig. 2.2). There are also a pseudo-thermodynamic measurements, where properties other than thermodynamic, but related to them, are measured. The early work on thin polymer films was performed primarily using the pseudothermodynamic methods (Alcoutlabi and McKenna, 2005). More recently dynamic measurements have been performed. In dynamic measurements the properties to be investigated are the viscosity and relaxation times as a function of temperature or frequency.

Some of the pseudo-thermodynamic measurements of $T_{g}$ that have been performed are Brillouin light scattering (Forrest and Dalnoki-Veress, 2001), x-ray reflectivity (van Zanten et al., 1996), ellipsometry (Fakhraai and Forrest (2005), Dalnoki-Veress et al. (2001), Singh et al. (2004), Sharp et al. (2004) etc.), positron annihilation spectroscopy (DeMaggio et al., 1997) and fluorescence probe intensity (Ellison et al., 2002). In addition, thermodynamic measurements on thin films have been performed using differential scanning calorimetry (Efremov et al., 2005). Forrest and Dalnoki-Veress (2001) presented some results in their famous diagram (see Fig. 5.1) showing a dramatic decrease in $T_{g}$ with reducing the film thickness.

The relaxation in thin films can be directly investigated using dynamic measurements. Thus Fukao et al. (Fukao and Miyamoto (2000), Fukao and 
Miyamoto (2001), Fukao et al. (2001) and Fukao (2003)) studied thin films by means of dielectric spectroscopy and thermal expansion spectroscopy. Kremer at al. (Hartmann et al. (2002), Serghei et al. (2005)) studied the $\alpha$ relaxation by means of dielectric spectroscopy. Both, Fukao and Miyamoto (2001) and Serghei et al. (2005), studied exactly the same system, thin PS films between two evaporated Al surfaces, but the dynamics observed differed from each other. A possible explanation for the different results can be found in Sections 5.1.1 and 5.1.3. Hall et al. (1997) investigated the $\alpha$ relaxation in thin films by second-harmonic generation, concluding that the relaxation time distribution broadens with reduced dimensions, but $\langle\tau\rangle$ remains constant within the error of the setup. However, the system studied consists of a complex polymer with a large side-chain on a patterned quartz substrate, hence it is hard to compare the system with the other results.

In the recent work of Fakhraai et al. (2005), the time needed for healing of thin PS bilayers in order to observe the segmental dynamics was measured by ellipsometry. They find that by reducing the thickness the healing time increases, revealing that the out of plane relaxation becomes slower. The $T_{g}$ measured on the same samples show the familiar behavior of a decrease with the thickness. Therefore they claim that for thin films the measurements of $T_{g}$ cannot be used to predict chain dynamics and vice versa. Furthermore, it is suggested that comparison between different measures of dynamics may not be valid. The explanation for that will be given in the further text. In any case the question "what happens to the dynamics in thin polymer films" remains opened.

This work is the first try in measuring the dynamics in thin polymer films by means of mechanical spectroscopy. One reason may be the demand of high sensitivity to measure the damping of very low amount of material. The importance and the advantage of the method compared to dielectric measurements are the presence of a surface of the studied thin polymer films. The free surface plays a major role in the investigation of thin films as it will be explained in Section 5.1.1. The influence of reduced dimensions on the dynamics of thin films was measured for (PS). The repeating unit of PS is depicted in Fig. 5.2. The term dynamics should be considered in the context of the short length scale motion associated with the glass transition and the diffusion of polymer chains. The material is throughly studied, providing the best chance to elucidate the problem of the glass transition. The atactic polystyrene cannot crystallize (see Section 2.2.1) and does not dewet the Si surface (DeMaggio et al., 1997). The decrease of the glass transition temperature of supported PS films is not dependent on the type of substrate (DeMaggio et al. (1997), Forrest and Dalnoki-Veress (2001)), as can be seen in Fig. 5.1. Compared to the measurements done on poly(methyl methacrylate) (PMMA), the polymer which is also often investigated, the magnitude of $T_{g}$ depression and the slope of $T_{g}$ versus the film thickness were higher for PS then for PMMA (Roth and Dutcher, 2003). 


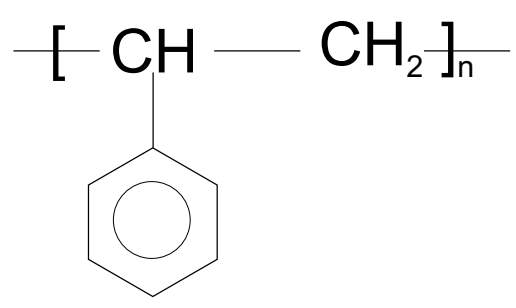

Figure 5.2: The chemical makeup of polystyrene is a long chain hydrocarbon with every other carbon connected to a Phenyl group.

Hence, the system PS on a Si substrate is optimal for the investigation of dynamic mechanical behavior of supported polymer films.

In the frame of this work the vibrating reed method is successfully applied, not only to measure $T_{g}$ but also to study changes in the $\alpha$ relaxation of thin polymer films with respect to the bulk.

\subsubsection{The influence of confinement on dynamics}

The effect of confinement on dynamics in thin films is throughly studied and the contradictions in results concerning glass transition have often been related to it. Hence the systems of polymer films confined between two free surfaces, between one hard (treated or not treated) and one free surface and two hard surfaces are still investigated. The main idea is that the free surface is crucial for observing the depression in $T_{g}$ by films of reduced dimensions due to a liquid-like layer of increased molecular mobility and to an increase of the free volume amount at the film - vacuum (or gas) interface. The role of the interface and the liquid-like layer is interesting because DeMaggio et al. (1997) found that the thermal expansion coefficient of PS films in the glassy state was independent of the film thickness, whereas in the liquid state it is found to decrease with decreasing the film thickness. Hence, the existence of a liquid like layer could cause a decrease in the amount of free volume in a thin film. However, for reptation-like dynamics (kinks may diffuse along a loop, if it is extended to the surface region) a lower amount of the free volume is needed (de Gennes, 2000).

An early report on the influence of the free surface on the glass transition temperature of thin polymer films was given by Forrest et al. (1996). They measured $T_{g}$ of freely standing thin PS films and compared the results to those of Keddie et al. (1994) on supported PS films.

In their recent study, Sharp et al. (2004) showed the high importance of the free surface by measuring different systems using ellipsometry. They compared systems of thin PS films with one free surface to the systems capped by a $5 \mathrm{~nm}$ thin Au layer and by a $5 \mathrm{~nm}$ thin Al layer. They showed that in the case of $\mathrm{Al}$ capped films a partial delamination of the metal layer can take place which leads to the free surface. This does not happen with the 
gold layer. In the case when two thin films are spin-coated on an evaporated $\mathrm{Al}$ layer and then connected and healed together, it is not possible to see the depression in the $T_{g}$. As soon as the $\mathrm{Al}$ layer is removed, the depression can be measured. The further explanation for such a behavior of $\mathrm{Al}$ and $\mathrm{Au}$ on a PS film can be found in (Sharp et al., 2004), (Faupel et al., 1998), and (Strunskus et al., 2000). These findings are important for explaining different results in dielectric measurements.

\subsubsection{The influence of the chain length on dynamics}

Experimental investigations of the molecular weight $\left(M_{w}\right)$ dependence of $T_{g}$ in free-standing thin polymer films showed that the $T_{g}$ depression with the $M_{w}$ reduction is very pronounced (Roth and Dutcher (1997), DalnokiVeress et al. (2001), Roth and Dutcher (2003)). However, for supported thin polymer films Singh et al. (2004) reported a decrease in $T_{g}$ measured by ellipsometry and Roth and Dutcher (1997) report no significant change measured by Brillouin light scattering and ellipsometry. Both systems studied were polystyrene on $\mathrm{SiO}_{\mathrm{x}}$, with the difference that in the case of Roth and Dutcher (1997) a $\mathrm{SiO}_{\mathrm{x}}$ layer was evaporated on a Si substrate and the PS film was water transfered after spin-coating and annealing and by Singh et al. (2004) the films were spin-coated on a native $\mathrm{SiO}_{\mathrm{x}}$ layer and annealed afterward. Hence, the reason for the different observations could be in the fact that the evaporated $\mathrm{SiO}_{\mathrm{x}}$ layer might be rougher than the native oxide layer. The molecules on a rough surface have more contacts with the substrate, what would lead to more hindrance in the molecular mobility and to the shortening of segments the movements of which are influencing the glass transition comparable to those in a bulk. In the bulk the glass transition is independent on the molecular weight for $M_{w}>5 \cdot 10^{4}$.

Dalnoki-Veress et al. (2001) studied more then 40 different freely standing PS films with 6 different molecular weights, reporting the dependence of the glass transition in thin films for molecular weights ranging from $5.75 \cdot 10^{5}$ to $9.1 \cdot 10^{6}$. For each $M_{w}$ the decrease in $T_{g}$ with reduced dimensions is observed, but the reduction in $T_{g}$ of those with the lower $M_{w}$ started by thinner films and the decrease was slower than of those with the extremely high $M_{w}$ (e.g. for $M_{w}=1.25 \cdot 10^{6}$, which is the closest to the $M_{w}$ of the polymer used in the frame of this work, the critical thickness was about $70 \mathrm{~nm}$ ). Singh et al. (2004) studied supported thin PS films of three different molecular weights. The trend of their results is in agreement with (Dalnoki-Veress et al., 2001), but they observed a deviation from the bulk $T_{g}$ earlier (for $M_{w}=1.571 \cdot 10^{6} \mathrm{~K}$, the critical thickness was about $\left.160 \mathrm{~nm}\right)$.

In this work two different systems of PS on polished Si substrates were studied, one commercial with the $M_{w}$ of $2.8 \cdot 10^{5}$ and a rather broad $M_{w}$ distribution (see Section 5.5) and one PS standard with the $M_{w}$ of $1.07 \cdot 10^{6}$ and a narrow $M_{w}$ distribution with the polydispersity index $M_{w} / M_{n}=1.06$ 
( $M_{w}$ is the weight average molecular mass and $M_{n}$ is the number average molecular mass). All the measurements on thin films are performed with the later in order to exclude the influence of the high polydispersity on dynamics in thin films. The PS with the broad $M_{w}$ distribution is due to its low price used for measurements of films on the polished substrates, needed to check whether the effect can be observed by means of the VRA.

\subsubsection{The influence of the heating (cooling) rate on dynamics}

The film thickness dependence of the $T_{g}$ values influenced by the cooling rate was recently studied by Fakhraai and Forrest (2005) by means of ellipsometry. They found that by increasing the cooling rate, the depression of $T_{g}$ becomes smaller until at cooling rates higher than $90 \mathrm{~K} / \mathrm{min}$ there is only a slight evidence for a film thickness dependent $T_{g}$ value. As explanation for the effect it was suggested that the cooling rates can be considered as an inverse of some relaxation time probed by the experiment, hence by a fast cooling rate, slow dynamics cannot be observed. Compared to the results reported in the literature, they find that their results explain the apparently contradictory experimental results that can be found.

Mechanical spectroscopy done within this work is performed with heating/cooling rate of $0.2 \mathrm{~K} / \mathrm{min}$, leaving enough time for the relaxation to take place. In the experiments the influence of different heating rates was not tested, since the experimental setup does not allow to change increasing the cooling/heating rate significantly.

The fact that the cooling rate and the free surface as well as the molecular weight have influence on dynamics and hence on the glass transition temperature emphasizes the importance of paying attention to these issues, by experiments and indicate the complexity of polymer systems with reduced dimensions.

\section{$5.2 \quad$ Spin-coating of thin films}

As described in Section 4.2 thin films are spin-coated on a Si substrates with a native oxide layer. A single layer of a thin film the mechanical spectrum of which is presented in Section 5.3.2 is prepared from a very dilute solution of $0.017 \%$ PS in toluene. Since the relaxation times are frequency dependent, it is very important to perform all the measurements with the same frequency for different film thicknesses. Therefore the films, the spectra of which are directly compared in Section 5.3, are all spin-coated on the same substrate. The different film thicknesses are achieved by spin-coating one layer on top of the other. The sample is annealed after each new layer at the temperature of about $380 \mathrm{~K}$ (slightly above $T_{g}$ ) for several hours. Fakhraai et al. (2004) and Forrest and Sharp (2003) studied the influence of such a layered structure on the glass transition. There was not any effect observed depending on 
whether the films were spin-coated as a single layer or if more layers were applied one onto the other, if the film was annealed above its glass transition temperature afterward.

The annealing is very important because the material created by spincoating can undergo approximately $14 \%$ volume change after the evaporation of solvent, which would lead to a large temperature shift (McKenna, 2000). Alcoutlabi and McKenna (2005) claim that the biaxial constraint of the substrate could possibly lead to yielding of the material and that the yielded material can be considered to be in a thermodynamic state different from that of the undeformed material. In this work the mechanical spectra for each film are measured twice, in order to prove whether the structure and stability of the films is influenced by further heating. The first run brings the additional drying and relaxing of the film and since the glass transition temperature of PS is about $363 \mathrm{~K}$ and each run is up to $430 \mathrm{~K}$ (see Fig. 5.6) with the heating rate of $0.2 \mathrm{~K} / \mathrm{min}$, the additional relaxation at $T>T_{g}$ takes place for more than 8 hours (the cooling down of the apparatus is included). The measured curves do not differ from each other, proving that further relaxing of stresses does not influence the mechanical spectra.

\subsection{Mechanical spectroscopy of thin PS films}

By means of mechanical spectroscopy the internal friction caused by movements of micro-structural units inside a material is measured. The internal friction in the material damps out mechanical oscillations.

In the case of polymers, segmental movements and sliding of chains during the glass-to-rubbery transition cause a relaxation process, the $\alpha$ relaxation. This relaxation process is frequency and temperature dependent. The measurement of the absorbed energy as a function of temperature, keeping the frequency constant, gives the mechanical loss spectrum.

The information about the mobility of polymer molecules obtained from the mechanical spectra helps to learn about the changes in the material with reduced dimensions.

\subsubsection{Mechanical spectra of thick polymer films}

Before studying properties of a material with reduced dimensions, an understanding of its properties in the bulk state must be developed. Therefore, first the mechanical spectroscopy of thick PS films, where the influence of the interfaces of a film with its surroundings is negligible, has to be performed. Since the vibrating reed apparatus described in Chapter 4 was never before used for measurements of polymer films, this is a good way to test the precision of the method. In the case of thick films the average dynamics in the material and the mechanical properties related to it are independent of the film dimensions. 


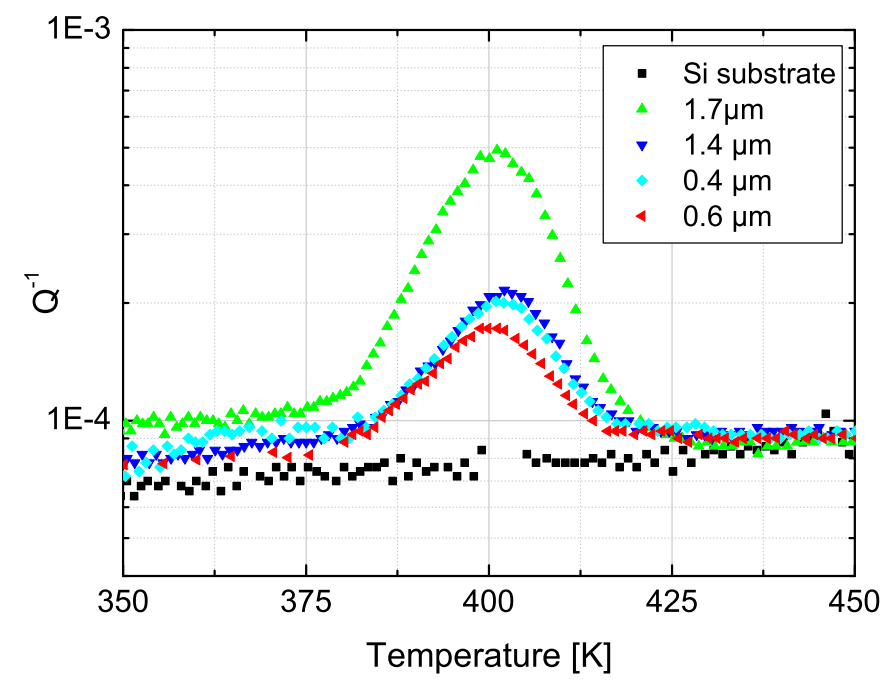

Figure 5.3: Mechanical spectra of composite samples including the glass transition peak (PS films on Si substrates) of different film thicknesses.

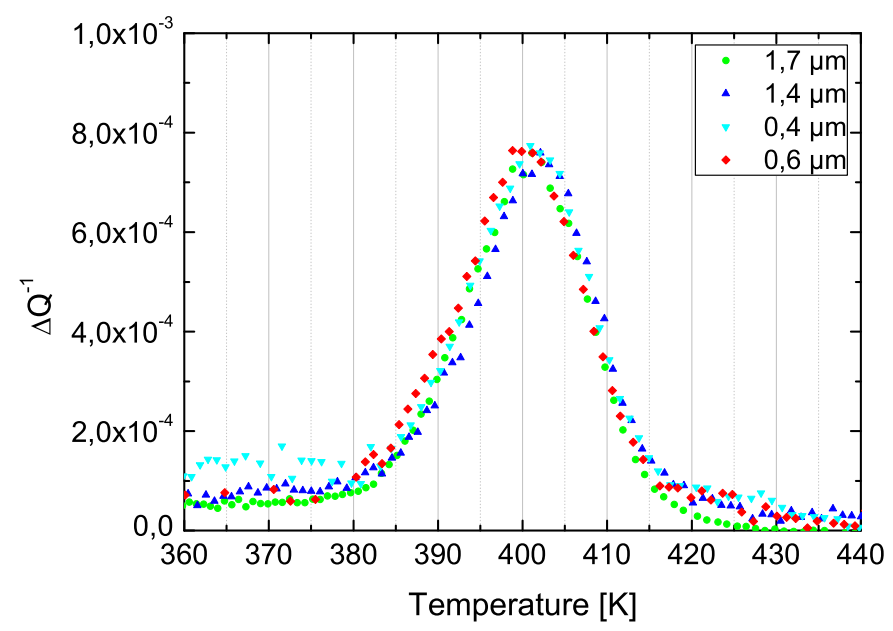

Figure 5.4: Subtracted and normalized mechanical spectra; in order to compare the relaxation peaks, the damping of the substrate has to be subtracted and the peaks have to be normalized to the same amplitude. It is obvious that in the case of thick films dynamics of molecules and thus the $\alpha$ relaxation is independent of the film thickness. 


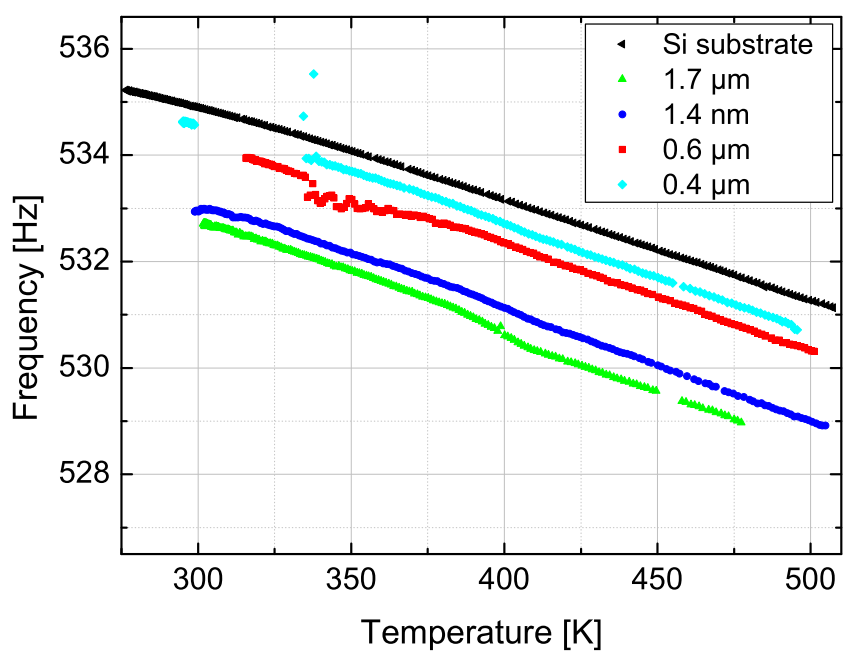

Figure 5.5: The eigenfrequency of the composite sample changes with film thickness. The frequency of the composite compared to that of the pure Si substrate yealds about the film thickness. The decrease in frequency is influenced by the decrease in modulus with increasing temperature. The inflection at about $400 \mathrm{~K}$, visible in the case of thicker films, results from the glass-to-rubbery transition. The "nonlinear" behavior at about $350 \mathrm{~K}$ in the case of the film of $0.6 \mu \mathrm{m}$ thickness, results from the offset of the laser beam from the detector and should be neglected.

The experiments on thick films presented in Figs. 5.3, 5.4 and 5.5 are performed with commercial PS with the $M_{w}=2.6 \cdot 10^{5}$ and a broad molecular weight distribution (see Section 5.5). The substrates used for these experiments are the polished cantilevers (see Section 4.1.1). The PS films are applied on a reed that is previously cleaned in methanol, toluene and acetone. All the spectra presented in Fig. 5.3 are measured with the same substrate in order to measure with the same frequency (the difference in the eigenfrequency due to the different film thicknesses is neglected). The heating (cooling) rate is $0.2 \mathrm{~K} / \mathrm{min}$ and it does not make any difference whether the sample is heated up or cooled down during the measurements.

In order to compare the spectra the damping of the substrate (and the other losses)is subtracted and the curves are normalized to the same amplitude. According to the results presented in 5.4 the spectra of the thick films are independent of the film thickness.

The film thicknesses are calculated by Eq. 3.20 from the shift in the eigenfrequency. The frequency of the sample is dependent on the film thickness as depicted in Fig. 5.5. The decrease in frequency with temperature is caused by the change in the elastic modulus of both materials. In the case 
of thicker films, the change in the modulus during the glass-to-rubbery transition at $T \approx 400 \mathrm{~K}$ can be observed in the frequency curve as an inflection.

\subsubsection{Mechanical spectra of thin polymer films}

The size of the regions over which cooperative structural rearrangements need to occur to allow a structural relaxation is called the correlation length. A polymer film is considered thin when the correlation length is of the same order of magnitude as the film thickness. Below a certain thickness, which is dependent on the molecular weight $M_{w}$ and the radius of gyration $R_{g}$, the confinement between two interfaces (solid substrate and free surface) restricts the free arrangement of the polymer chains and deviations from unperturbed coil conformation are expected. Decreasing the film thickness down to the order of the unperturbed chain dimension increases the in-plane length of the chains (Kraus et al. (2000), Russel et al. (1999)).

Dynamics of such a film differs from that of thick films. Below the critical thickness the average dynamics becomes faster. For supported PS films, the critical thickness is about $100 \mathrm{~nm}$ and it is independent of the substrate (Forrest and Dalnoki-Veress, 2001). Furthermore, the theoretical calculations Mason and Sutton (2005) and experiments (Gautam et al., 2000) on polystyrene showed that at both interfaces the preferred orientation of aromatic rings are out of the interface plane, at the film/substrate interface though, the tilt angle is higher than at the free surface.

In this section, first the best results on thin films, measured on a wet etched substrate (see Section 4.1.1), are presented and later in this section the less successful results on thin films with the other substrates are shown in order to show their limits.

\section{Spectra of films on wet etched substrates}

Different film thicknesses of the spectra depicted in Fig. 5.6 are achieved by spin-coating several layers on a wet etched substrate as described in Section 5.2. The eigenfrequency of the substrate is $100 \mathrm{~Hz}$. For each layer the measurement is repeated in order to prove the film stability. For all the films both the heating and the cooling cycles were performed and there is not any difference observed between the resulting spectra. The mechanical spectra are fitted to the Gauss function in order to make the comparison easier (Figs. 5.7 and 5.8). The simple Gaussian or lognormal distribution is a common mathematical function for describing mechanical spectra due to its symmetry and capability to describe the relaxation peak also in the case of a single relaxation time (San Juan, 2001). Two extreme cases of films, $7.5 \mathrm{~nm}$ and $95 \mathrm{~nm}$, are depicted in Fig. 5.9 and the Gauss fits of the spectra are normalized to the same amplitude. A clear broadening of the relaxation peak and its shift to the lower temperatures with reduced 


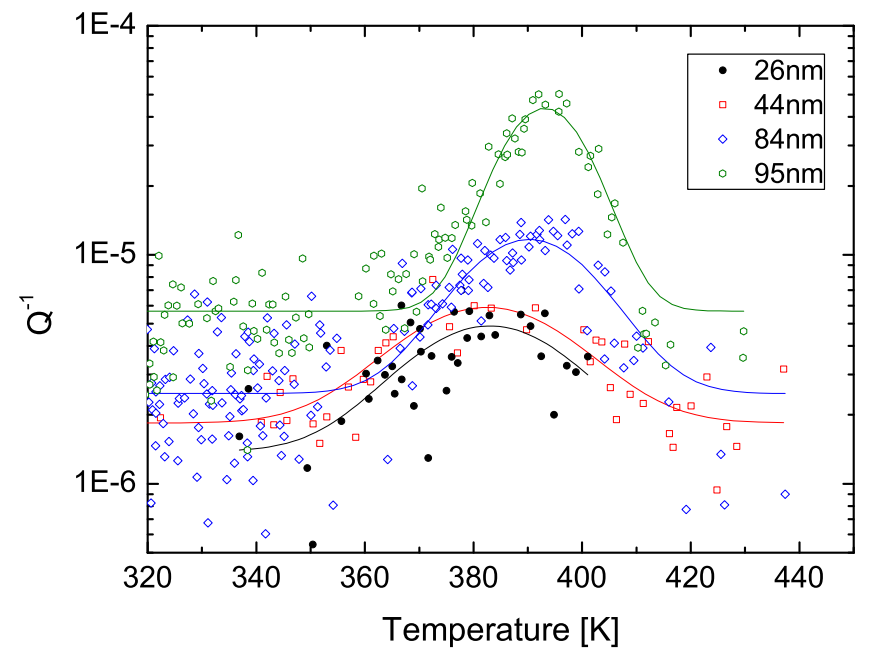

Figure 5.6: Mechanical spectra of thin PS films. The relaxation peaks are fitted to the Gauss fit. With reduced dimensions, the $\alpha$ relaxation onset and the peak maxima shift to the lower temperatures than in the case of thick films. The relaxation becomes broader due to the broader relaxation time distribution.

dimensions is observed. The film thickness is calculated from the frequency shift (Eq. 3.20). An example of the frequency shift is depicted in Fig. 5.11, where the measurement on the same film is performed three times in order to prove whether there was any residual solvent left in the film or some structural changes that could influence the elastic modulus and thus the frequency, too.

The spectra presented in Fig. 5.10 using another substrate at a frequency of $200 \mathrm{~Hz}$. For film thicknesses between 127 and $731 \mathrm{~nm}$ there is no further shift in the relaxation peak noticed. The spectra are not directly compared to those in Fig. 5.6 because the measurements are performed at the different frequency (the higher the frequency, the higher is the peak temperature).

\section{Spectra of films on polished substrates}

The effect of the reduced dimensions can also be observed using the 100 $\mu \mathrm{m}$ polished substrates (Figs. 5.12 and 5.13), though a part of the relaxation is covered by the damping of the substrate and, as depicted in Fig. 5.14 , it is not possible to calculate the film thickness from the shift in the eigenfrequency. The shift in the maximum is less then $5^{\circ} \mathrm{C}$ comparing to the spectrum of a thick film measured with the same substrate (Fig. 5.13). Compared to Fig. 5.6, a shift of $5^{\circ} \mathrm{C}$ gives a rough estimation of the film thickness of $80 \mathrm{~nm}$. The $M_{w}$ distribution of the PS used for the measure- 


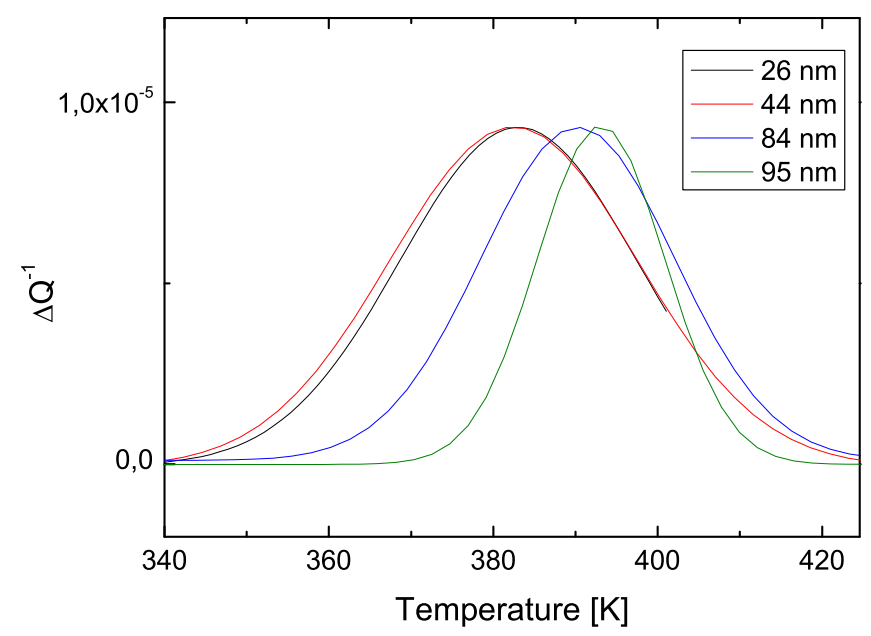

Figure 5.7: The Gauss fits of thin films mechanical spectra normalized to the same amplitude. The peak shift to the lower temperatures is caused by enhanced molecular mobility. The peak position and its width for the films of $26 \mathrm{~nm}$ and 44 $\mathrm{nm}$ thicknesses do not differ significantly.

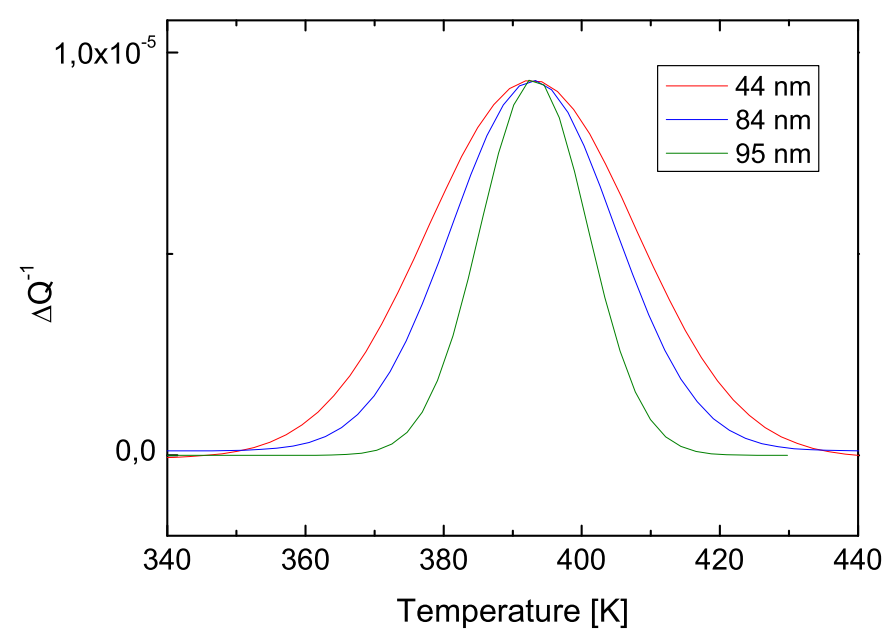

Figure 5.8: The Gauss fits of thin films mechanical spectra normalized to the same amplitude and to the same peak position of the $95 \mathrm{~nm}$ thick film. The relaxation peak width increases due to the broader relaxation times distribution in the case of thin films. 


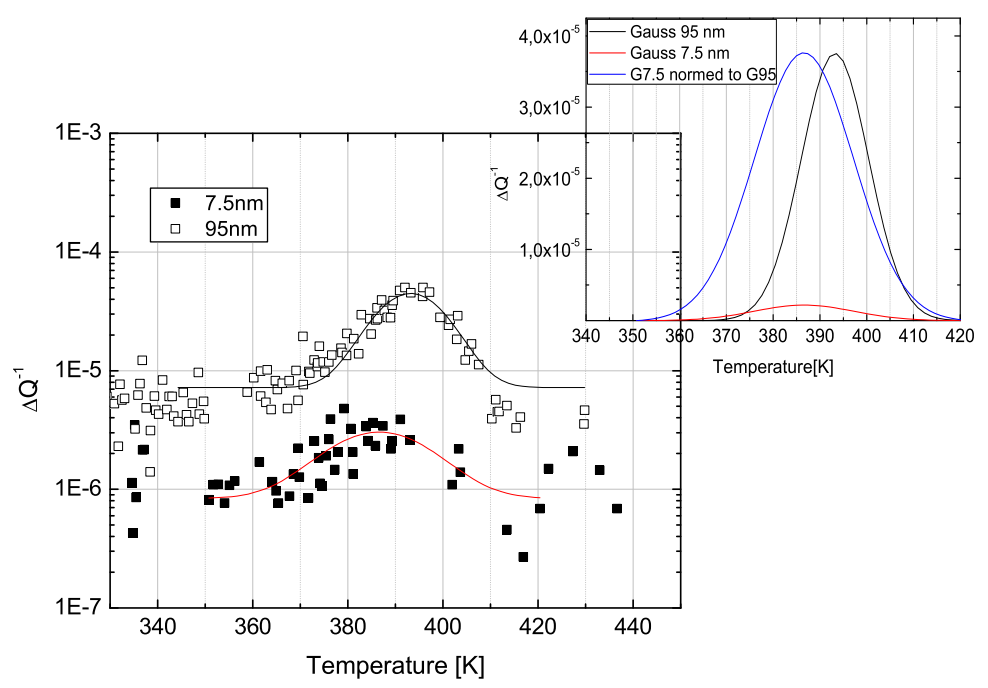

Figure 5.9: The thinnest film of $7.5 \mathrm{~nm}$ measured in the frame of this work compared to the $95 \mathrm{~nm}$ thick film.

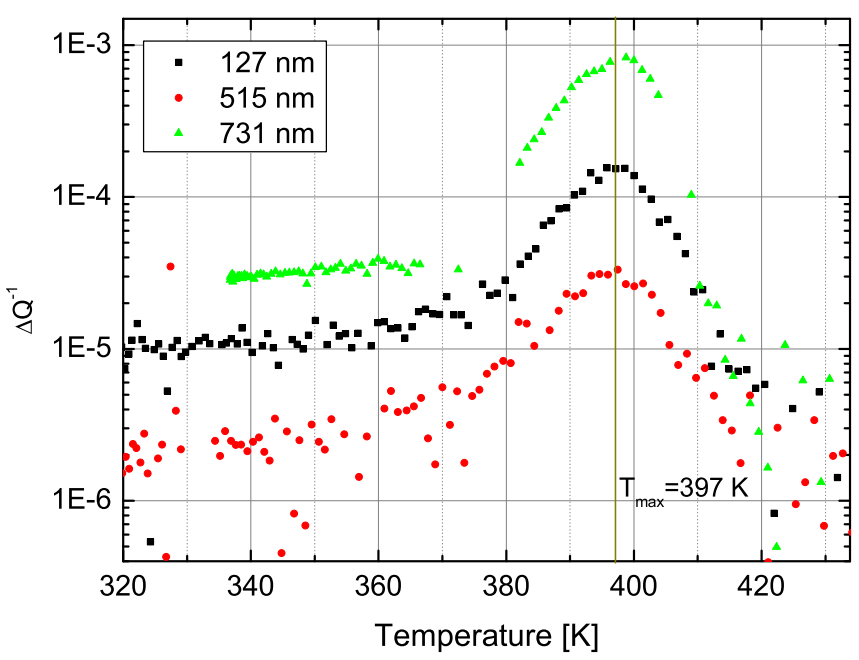

Figure 5.10: Mechanical spectra of thick films measured by the wet etched substrate. The relaxation peak is not influenced by the increase in film thickness. The spectra are not directly compared to those in Fig. 5.6 because the measurements were not performed by the same substrate. The eigenfrequency of the substrate used for measurements in Fig. 5.6 was $100 \mathrm{~Hz}$ and here it was $200 \mathrm{~Hz}$. This causes a slight shift in the peak maximum. 


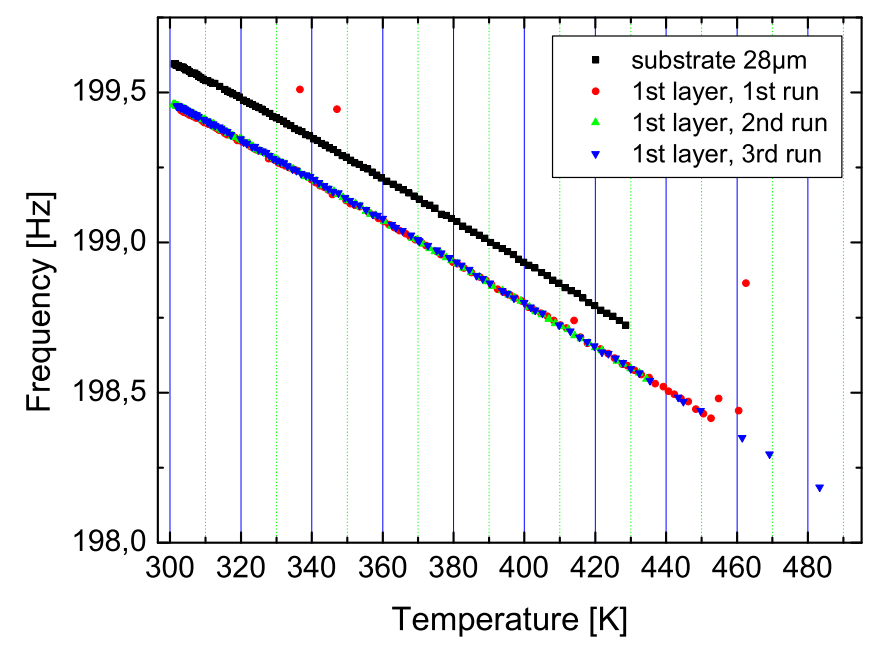

Figure 5.11: An example of frequency shift of a composite sample, the wet etched substrate and a thin film on it, compared to the frequency of the pure substrate. During the three runs, there was not any further shift in frequency proving that the solvent evaporated during the annealing time.

ments on this substrate is presented in Fig. 5.23.

\section{Spectra of films on dry etched substrates}

With the same polystyrene of a broad molecular weight distribution mechanical spectroscopy on films of different thicknesses is measured on the dry etched substrate. The results are depicted in Fig. 5.15. Again the relaxation process is pronounced and the shift in maxima and in the onset of the relaxation follow the same trend as described before. However, here a pre-peak appears, the origin of which is not clear.

The EDX measurements proved that after dry etching, $\mathrm{Fe}, \mathrm{Cr}$ and $\mathrm{Ni}$ atoms from the stainless steel remain on the surface of the substrate (see Appendix A). The polymer measured has a broad $M_{w}$ distribution and the first peak in Fig. 5.23 can be attributed either to monomers and dimers or to some impurities. Both effects can influence the result in an unpredictable manner. Additionally, the eigenfrequency does not shift to lower values with increasing the film thickness. This effect can only be explained if some further loss in weight, probably due to the evaporation in vacuum occurred. Hence, it is not possible to get any information about the film thickness.

The position of the pre-peak is thickness independent and the onset of the relaxation is at $360 \mathrm{~K}$. The peak is not attributed to the $\beta$ relaxation because, as already mentioned, McCrum et al. (1991) claims that the $\beta$ relaxation of 


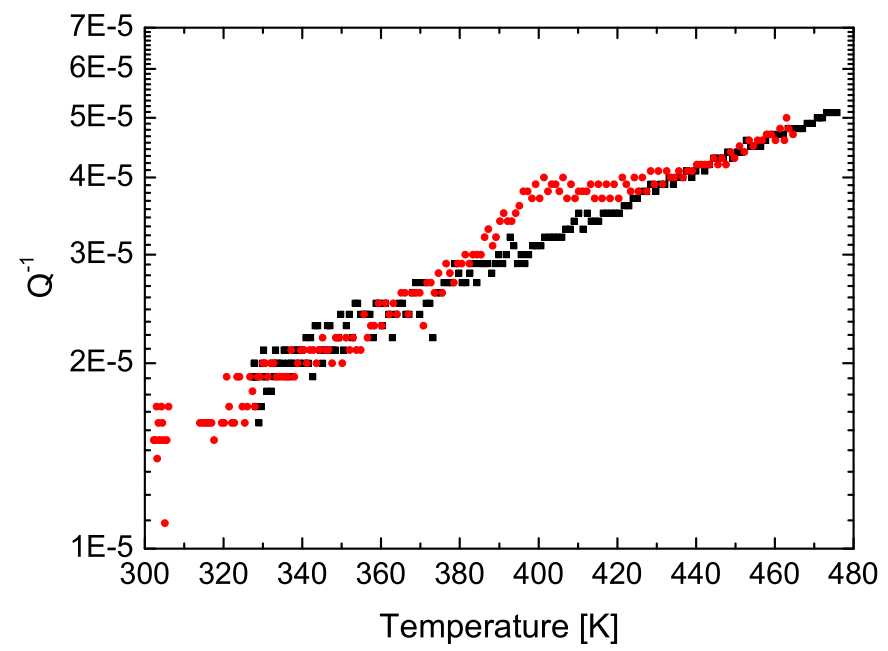

Figure 5.12: The mechanical spectrum of a thin film on the $100 \mu \mathrm{m}$ thick Si substrate. The relaxation onset might not be observed due to the high damping of the substrate.

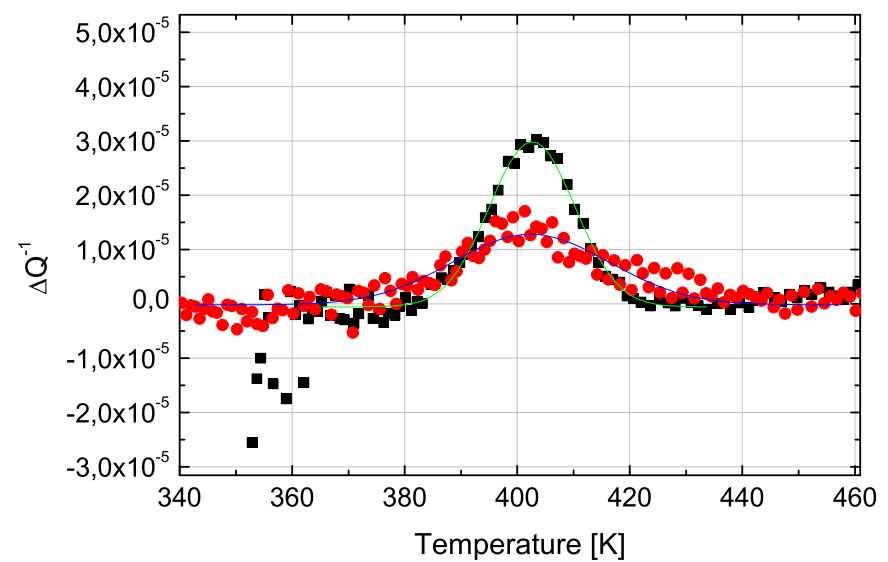

Figure 5.13: Subtracted spectra of a thick and a thin PS film are fitted to the Gauss function. The trend in the peak shift and in the width of the relaxation peak is the same as observed for the spectra of films measured on a thin wet etched substrates (Fig. 5.6). 


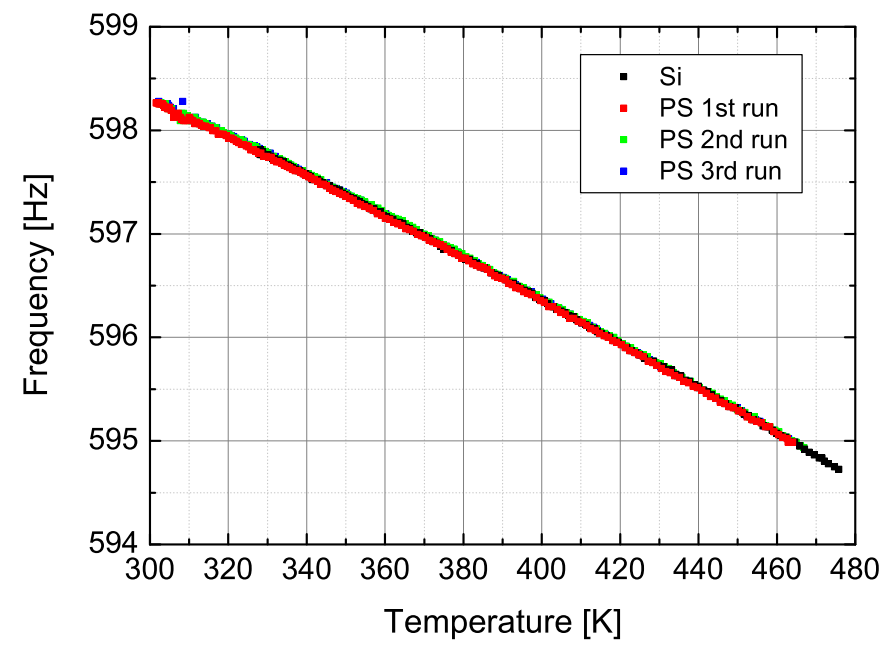

Figure 5.14: The frequency of the pure $100 \mu \mathrm{m}$ substrate does not differ from that of the composite sample, hence it is not possible to calculate the film thickness.

PS merges for frequencies higher than $40 \mathrm{~Hz}$. The slow $\gamma$ relaxation is in the case of polystyrene reported to be at very low temperatures of about 50 $\mathrm{K}$. The origin of the pre-peaks remains unclear.

\subsubsection{Effect of the film damping on the damping of the com- posite}

Equation 3.51 relates the data measured with a composite sample with the mechanical properties of the substrate and of the film below $T_{g} . \Delta Q^{-1}$ increases linearly with the film thickness $d_{f}$. Since the measurements are performed with substrates of different thicknesses $d_{s}$, it is more convenient to present the dependence of $\Delta Q^{-1}$ on the ratio $d_{f} / d_{s}$. The results for the glassy state, $T<T_{g}$, are presented in Fig. 5.16.

The linear fit of the function

$$
\Delta Q^{-1}=\frac{3 E_{f} d_{f}}{E_{s} d_{s}} Q_{f}^{-1} \equiv C \frac{d_{f}}{d_{s}}
$$

yields the coefficient $C=5.85 \cdot 10^{-4} \pm 3.16 \cdot 10^{-5}$. The expected slope $C$ of the linear function can be calculated as $\frac{3 E_{f}}{E_{s}} \cdot Q_{f}^{-1}$. Hiki and Kosugi (2005) measured the dependence of quality factor of $\mathrm{PS}, Q_{P S}^{-1}$, on temperature and frequency. At $\mathrm{T}=297 \mathrm{~K}$, the quality factor is weakly dependent on frequency and varies between 0.01 and 0.012. Although Hiki and Kosugi (2005) measured at frequencies between $0.001 \mathrm{~Hz}$ and $10 \mathrm{~Hz}$, it is assumed 


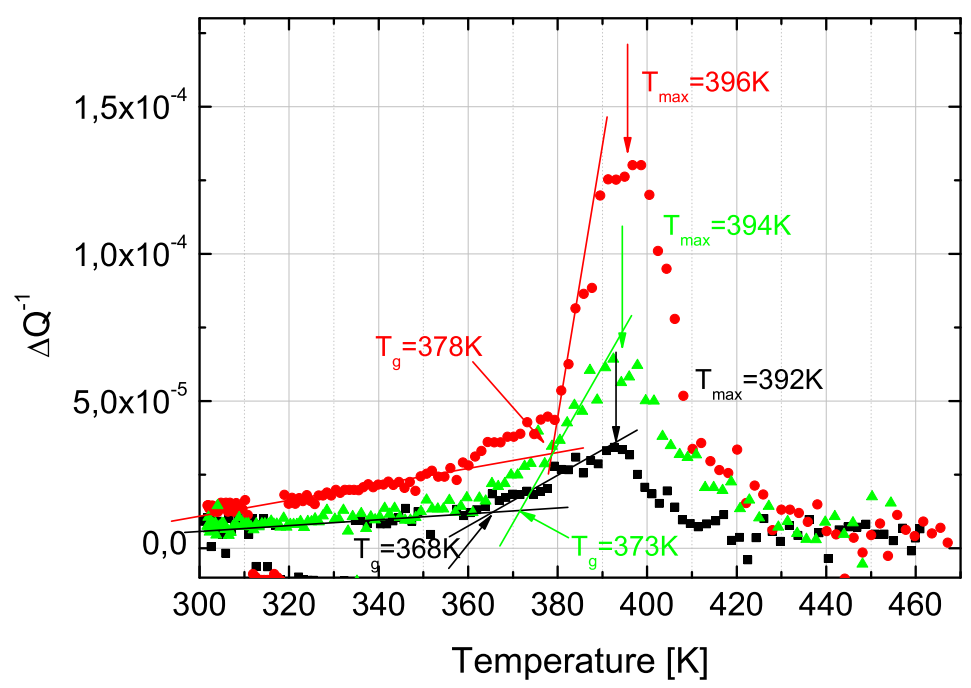

Figure 5.15: Mechanical spectra of films after background subtraction with different thicknesses spin-coated on a dry etched substrate. Though the relaxation peak shifts to lower temperatures and the relaxation time distribution becomes broader, the peak is not symmetric and a pre-peak, the position of which is independent of the film thickness, occurs. The origin of the pre-peak is not clear. The difference in film thickness is achieved by spin-coating the films from different solutions. Unfortunately, as described in the text, it was not possible to calculate the film thicknesses from the frequency shift. 


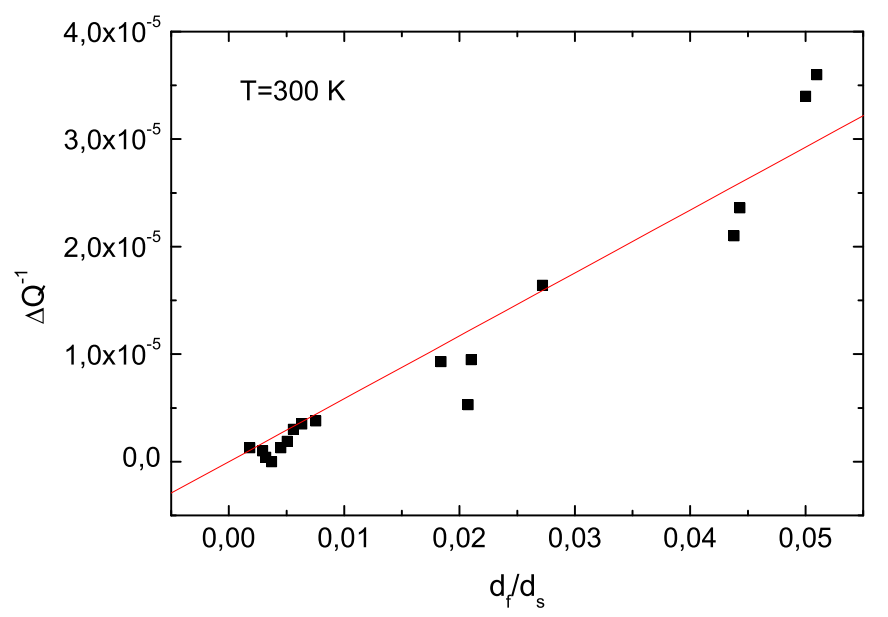

Figure 5.16: Dependence of the damping of the composite on the film thickness at $300 \mathrm{~K}$.

that at such a low temperature there will not be any dramatic change at higher frequencies, because the viscous response at low temperatures occur at low frequencies. Hence, with $Q_{f}^{-1}=Q_{P S}^{-1} \approx 0.01, E_{f}=3 \cdot 10^{9} \mathrm{~Pa}$ (Van Krevelen, 1990) and $E_{s}=163 \cdot 10^{9} \mathrm{~Pa}$, the slope is calculated as $C=5.52 \cdot 10^{-4}$. Thus the result measured by means of the VRA experiment is in good agreement with the literature data.

The offset from the linearity occurs either when a film is too thin for a substrate and at $T<T_{g}$ it is not possible to distinguish the damping of the pure substrate from that of the composite sample (e.g. Fig. 5.12) or in the case when the films are too thick for the substrate resulting in measurements that are a kind of unstable. The second case might occur due to the bending of a sensitive Si substrate caused by the mass of the film.

Hence, the diagram in Fig. 5.16 gives us two important information, one is the experimental confirmation of Eq. 3.51 and the other one is about the limits of the experiment.

\subsubsection{Film thickness measured by FIB/SEM}

The vibrating reed method allows not only to measure the internal friction of the sample, but also its thickness from the eigenfrequency of the sample. However, the situation with supported polymer films is a bit more complicated than in the case of a simple reed. Therefore the thicknesses calculated using Eq. 3.20 are proved by the FIB/SEM analysis. The samples are prepared as described in Section 4.3, from the same solution as those measured 


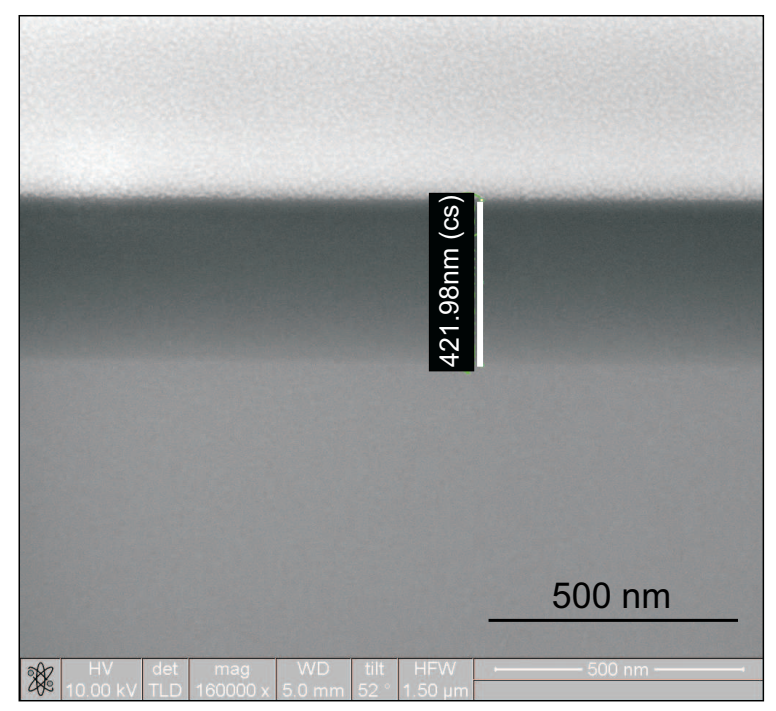

Figure 5.17: A cross-section of a thick film, the thickness of which was known from the color of the film (the so called Newtonian rings; the thickness is equal to the half of the wavelength of the film color).

by the VRA with the same spin-coating parameters. However, substrates are of different geometry, what may affect the calculated film thickness. However, the thicknesses measured by both methods are comparable. The films spin-coated on larger substrates for the FIB preparation are $20 \%$ to $30 \%$ thicker of those spin-coated on the vibrating reeds.

Not all the films measured by FIB/SEM are presented here due to the limits in contrast, specially in the printed version. Representative results showing films of several thicknesses, as well as the critical, the thinnest film, are presented in Figs. 5.17 to 5.20. In Fig. 5.18 the deposited Pt layer and the milling of the cross-section can be seen. From Fig. 5.18 it is obvious that for the shown film, the thickness of which is between 6 and $13 \mathrm{~nm}$ the limits of the FIB/SEM film characterization are reached as well as the ones of spin-coating and of the VRA (see Fig. 5.9). The last Fig. 5.21 presents the cross section of silicon/film composite which was cooled down to liquid nitrogen temperature and broken afterward. The descriptions are given in the captions with the references to the films measured by the VRA prepared in the same way.

\subsection{Activation energy}

In the field of solid-state physics and material science a variety of relaxation phenomena of the thermal activation type are observed. In relaxation an element, which can be an atom, a molecule or a group of either, tries to surmount a potential-energy barrier with some rate. The barrier height 

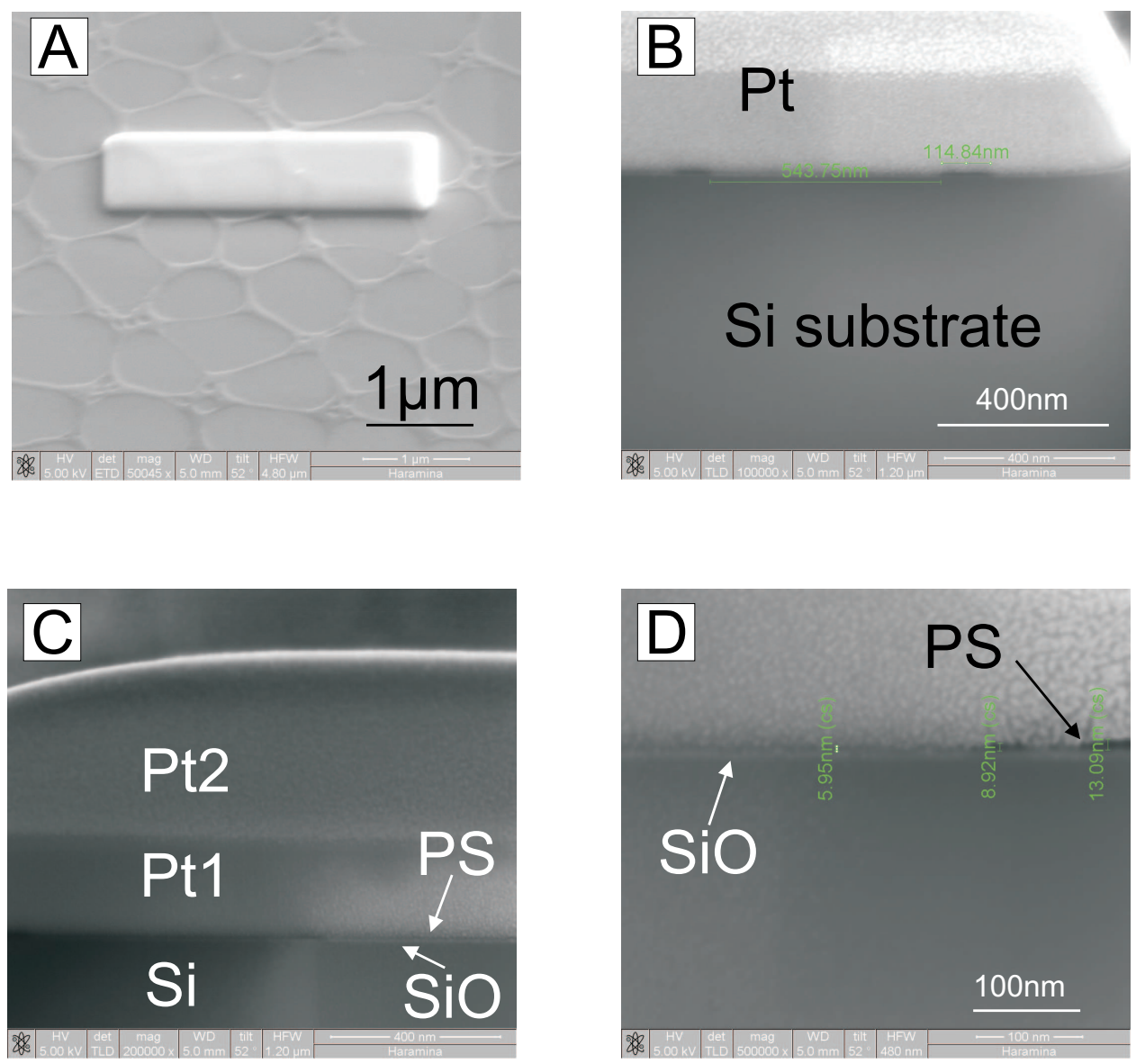

Figure 5.18: Pictures of the film surface and the FIB preparation of the crosssection. The film prepared from the same solution and the same spin-coating parameters as that of the film, the spectrum of which is depicted in Fig. 5.9. In Fig. A the surface of the thin film can be observed. A small area is covered by Pt. Although the surface is rough, the amount of the "hills" is small comparing to the area of the "valleys", therefore, the thickness of the "valleys" is closer to the average film thickness. In Figs. B, C and D the cross-section can be seen. Starting from below, the cross-section consists of the Si substrate (dark gray), a few nanometers $\mathrm{SiO}$, the PS film, Pt deposited by the electron beam and Pt deposited by the ion beam. The film thickness vary between 6 and $13 \mathrm{~nm}$. The diameter of the "valleys" of $6 \mathrm{~nm}$ are 500-600 nm, whereas the width of the "hills" of are up to $120 \mathrm{~nm}$. The thickness of the film prepared from the same solution, but on the substrate of different geometry, calculated from the eigenfrequency shift is $7.5 \mathrm{~nm}$. 


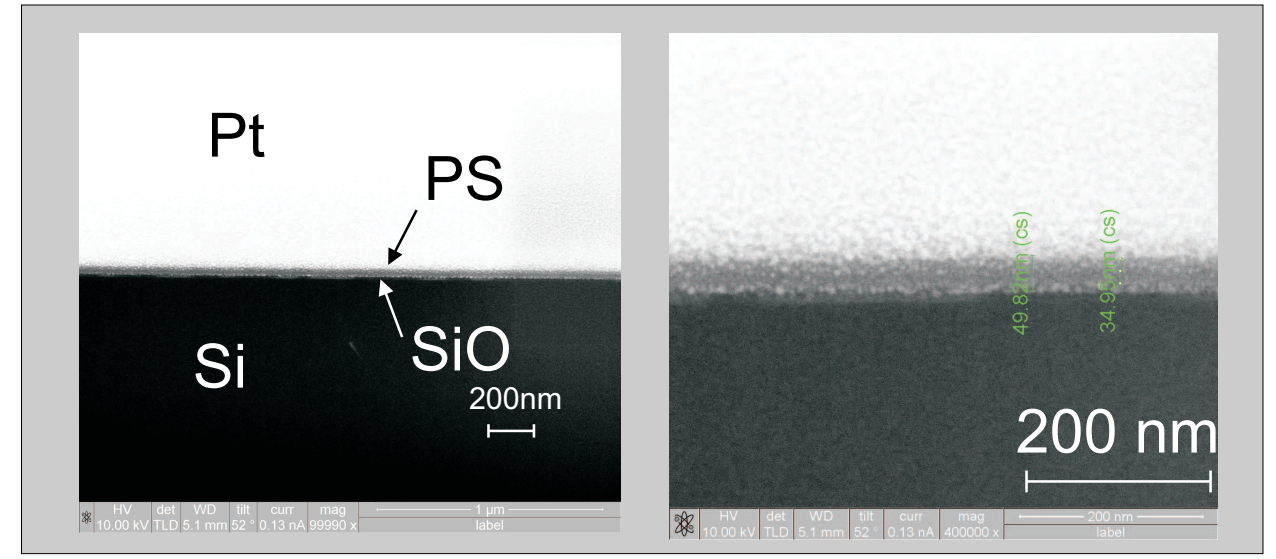

Figure 5.19: The $35 \mathrm{~nm}$ thick film made of three spin-coated PS layers is annealed at $T>T_{g}$ after each spin-coating. The preparation is the same as that of the 26 $\mathrm{nm}$ film (calculated value from frequency shift), the mechanical spectrum of which is depicted in Fig. 5.6.

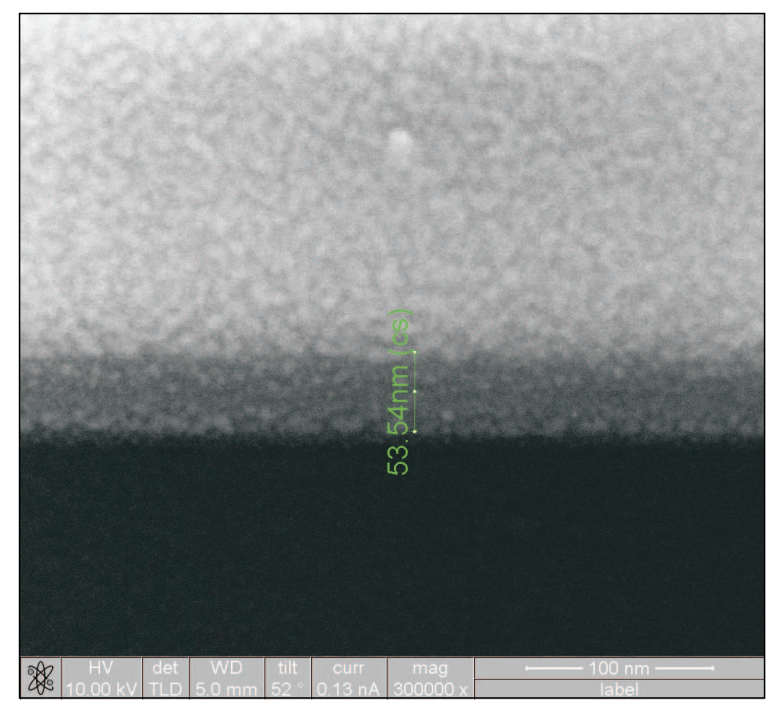

Figure 5.20: This $53.5 \mathrm{~nm}$ thick film has one layer more than that in Fig. 5.19 and is comparable to the $44 \mathrm{~nm}$ thick film (calculated value from frequency shift), the mechanical spectrum of which is depicted in Fig. 5.6. 


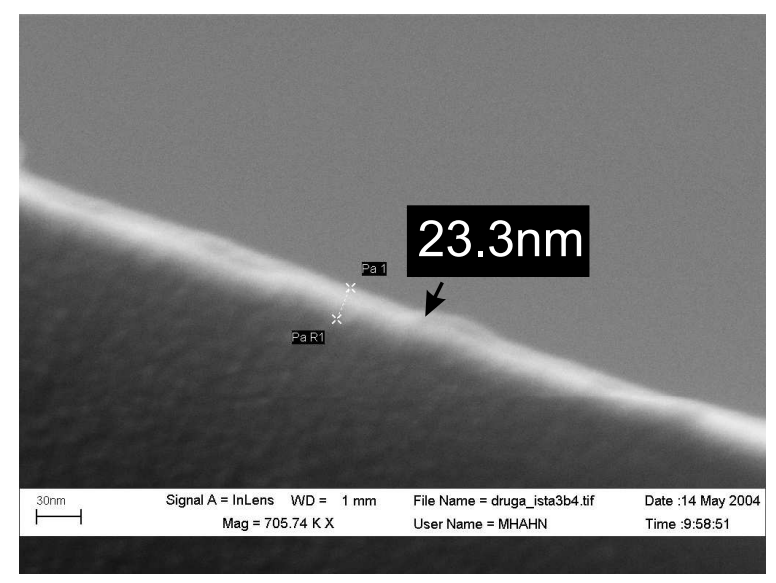

Figure 5.21: SEM micrograph of the cross section of a silicon/film composite which was broken after cooling in liquid nitrogen. The film prepared was prepared from the same solution as the film, the mechanical spectrum of which is presented in Fig. 5.12. The thickness of $26 \mathrm{~nm}$ was confirmed by ellipsometry done at the company Nanofilm, Göttingen.

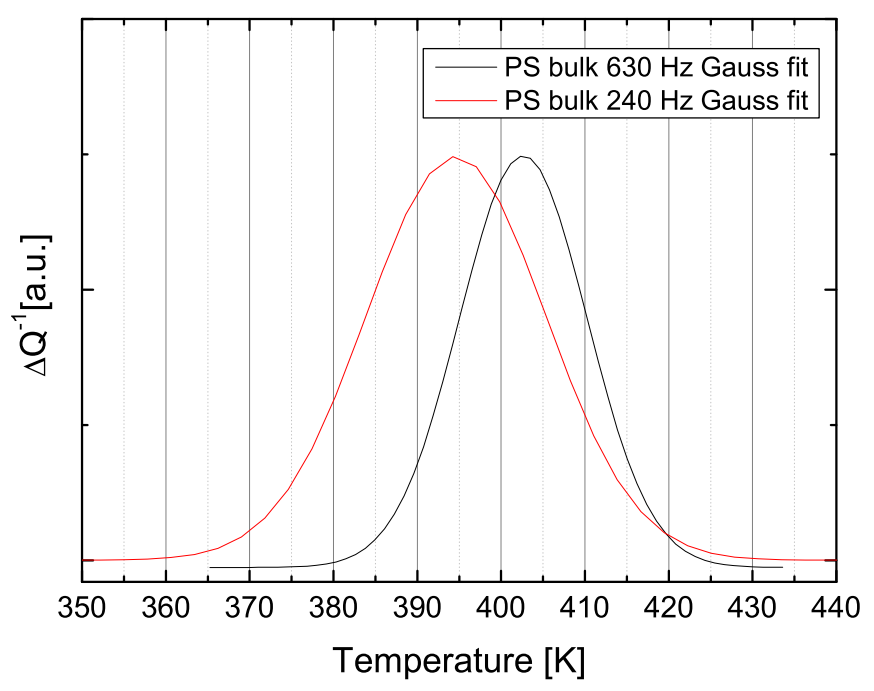

Figure 5.22: The $\alpha$ relaxation measured at two different frequencies gives information about the activation energy. The different width of the peaks is caused by high damping of the thicker substrate, the damping of which, therefore, covers a part of the relaxation peak. 
that must be overcome in the course of hopping from one potential well to the next is called the activation energy $Q_{a}$ and in turn it gives one an idea of the energetics of the particular molecular process.

The energy can be determined by mechanical spectroscopy at different frequencies. The lower the frequency, the lower will be the temperature where the $\alpha$ relaxation has its maximum.

From Eq. 2.4 the activation energy can be calculated as

$$
Q_{a}=\left(R \ln \frac{\omega_{2}}{\omega_{1}}\right)\left(\frac{1}{T_{1}}-\frac{1}{T_{2}}\right)^{-1} .
$$

From two spectra measured at different frequencies (Fig. 5.22), the activation energy calculated is $Q_{a}=160 \mathrm{~kJ} / \mathrm{mol}$. In the calculation $T_{1}$ and $T_{2}$ are the temperatures at the peak maxima. It is possible, though, to do the calculation at any temperature (Nowick and Berry, 1972), but this introduces an additional inaccuracy due to the error in subtraction of the background. The peak position depends on the molecular weight; therefore the films, the relaxation spectra of which are compared, must be of the same polymer.

The value calculated is close to $130 \mathrm{~kJ} / \mathrm{mol}$ found in the literature (Kaliappan and Cappella, 2005), but one must be aware that the peak is rather broad, what makes the estimation of the peak temperature problematic. A better result would be achieved if the measurements were done at a frequencies that differ for at least one order of magnitude.

\subsection{Molecular weight distribution measured by Size Exclusive Chromatography (SEC)}

The PS of the average molecular weight $M_{w}=2.8 \cdot 10^{5}$ was purchased from Aldrich. Since the polymer had the unknown molecular weight distribution, the analysis is done at the Institute of Physical Chemistry, Göttingen. The result of the analysis is presented in Fig 5.23. The distribution is rather broad spanning from $10^{4}$ to $10^{6}$. The small peak at low molecular weight results either from monomers and dimers or from some impurities in the material and the SEC apparatus. The thin films measurements in Section 5.3.2 which are very sensitive to the $M_{w}$ are not performed with this polymer.

\subsection{Discussion}

The effect of reduced dimensions on the $\alpha$ relaxation process is successfully measured by means of mechanical spectroscopy. The vibrating reeds enable measurements with a free surface, which gives the method an advantage compared to dielectric loss measurements. The low heating/cooling rate enables the measurements of the decay at nearly constant temperature and 


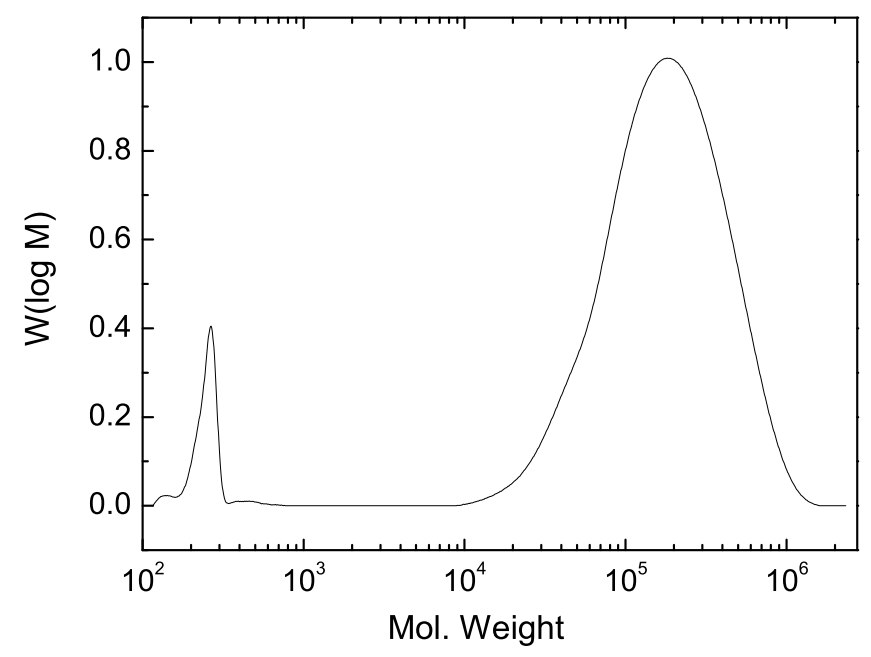

Figure 5.23: Molecular weight distribution of the commercial PS with $M_{w}=2.8 \cdot 10^{5}$ measured by Size Exclusive Chromatography.

the relaxation time probed by the experiment is long enough for observing the effects of the reduced dimensions (see 5.1.3). The PS measured has a high $M_{w}$ ensuring strongly entangled structure.

The $\alpha$ relaxation in thick polymer films is independent of the films thickness and behaves like bulk. Below a certain thickness the peak becomes broader and it shifts to lower temperatures. The increased width of the peak stems from the broader relaxation time distribution, because the dynamics during the relaxation in thin films differs from that in the bulk polymer films. The lower energy needed for the relaxation to take place enables the movements of molecules at lower temperatures, observed as the peak shift.

However, as it can be seen in Figs. 5.24 and 5.25, the changes in the relaxation peak are obvious for thin films of $84 \mathrm{~nm}$ and $44 \mathrm{~nm}$ thickness compared to the bulk $95 \mathrm{~nm}$ thick film, but with the further thickness reduction only the relaxation strength, related to the decrease in the amount of material, is influenced.

For the sake of comparison the Gauss fits from Fig. 5.6 are depicted in Fig. 5.24. In Fig. 5.25 the effect of reduced dimensions on the peak maximum and the relaxation onset defined in Fig. 5.24 is shown.

The existence of a liquid like layer on the surface of polymers is experimentally confirmed and its thickness is $5 \pm 1 \mathrm{~nm}$ (Sharp et al., 2004). The molecules which extend to the surface may diffuse as described by de Gennes (2000) and the substrate/film interface may influence dynamics in the opposite way. During mechanical spectroscopy the liquid-like layer will response 


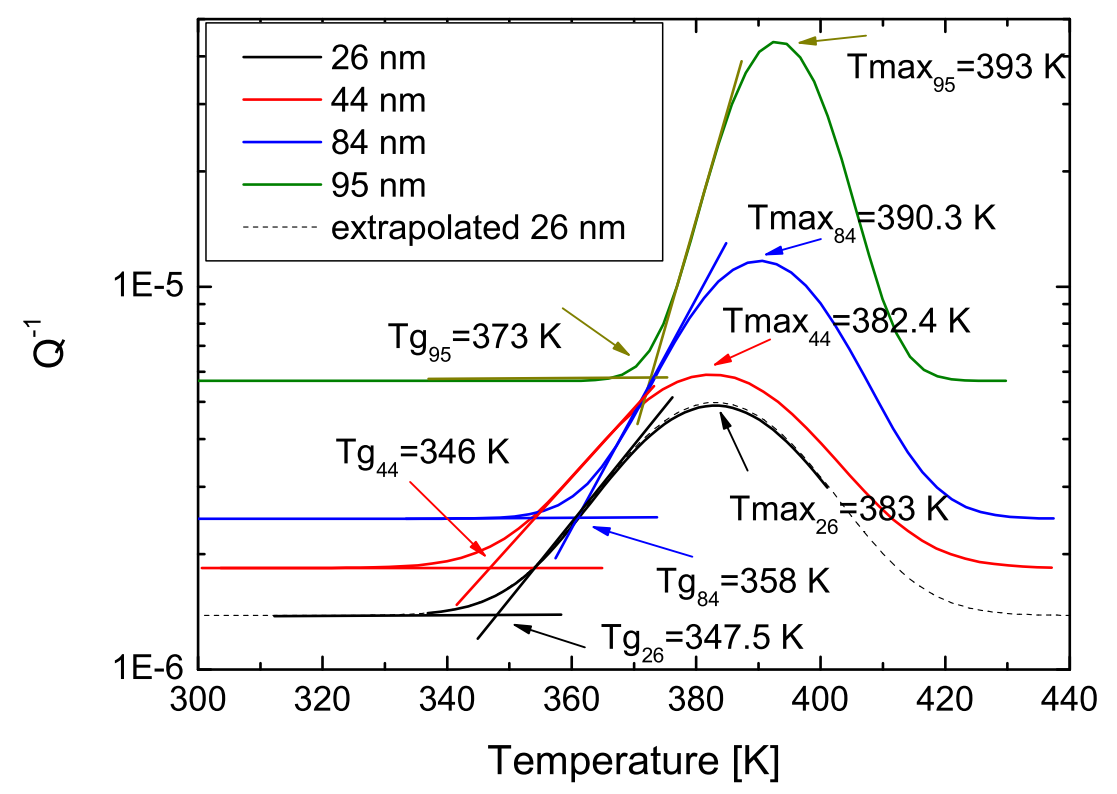

Figure 5.24: Gauss fits of the data presented in Fig. 5.6. The temperatures given in the spectra are the peak maxima, $T_{\max }$, and the relaxation onsets in the mechanical spectroscopy marked as $T_{g}$ defined as the intersection between linear fit at low temperatures and the tangent at the inflection point (convention). The stronger shift in the $T_{g}$ than that of the $T_{\max }$ is due to the broadening of the relaxation time distribution.

in a viscous manner and therefore the layer does not undergo the glass transition at the measuring temperatures. As a consequence the glass transition temperature should approach zero if the film thickness reduces to the thickness of the liquid-like layer. There must be a counteracting effect, in order to explain the experimental results shown in Fig. 5.25.

The orientation of the aromatic ring at the substrate/film interface from the substrate into the film with preferred tilt angle of $70^{\circ}$ causes an increase of the density at that interface, what inhibits the movements of molecules. If the thickness of the layer influenced by the density increase is comparable to that of the liquid-like layer (i.e. $5 \mathrm{~nm}$ ), a film of $10 \mathrm{~nm}$ would consist of a liquid like layer and the layer of higher density (solid-like layer). Independent of the thickness of the solid-like region at some film thickness the increase in the molecular mobility due the reptation equals the inhibited movements due to the increase in density. In this work the thickness where no further depression in the relaxation temperature is noticed is about 44 $\mathrm{nm}$. Although it seems that the relaxation peaks of films of $26 \mathrm{~nm}$ and 7.5 


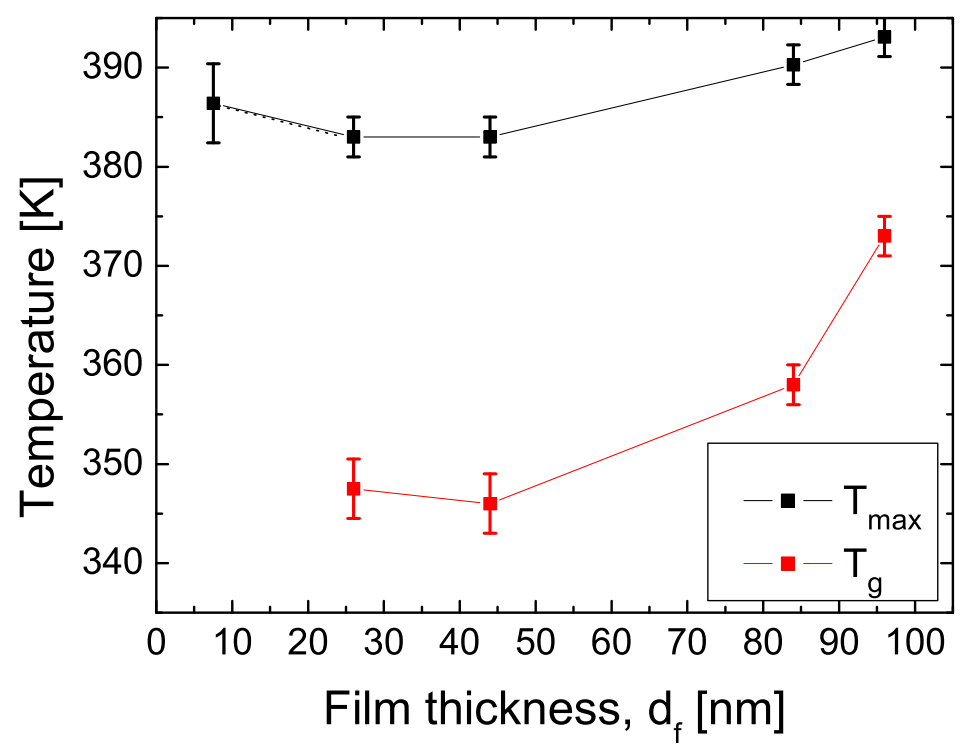

Figure 5.25: Glass transition temperature $T_{g}$ and maximum $\alpha$ relaxation temperature $T_{\max }$ as given in Fig. 5.24. The estimated error in thickness $\pm 5 \mathrm{~nm}$. The data of the film of $7.5 \mathrm{~nm}$ thickness (Fig. 5.9), is noise limited. Nevertheless, it is obvious that there is no further depression in the relaxation peak below $44 \mathrm{~nm}$ film thickness.

$\mathrm{nm}$ are even slightly shifted to the higher temperatures, these departures are within the uncertainties of the data.

The data from Fig. 5.25 is compared in Fig. 5.27 with the one from Fig. 5.1. Both temperatures, $T_{\max }$ and $T_{g}$, decrease with the films thickness down to $44 \mathrm{~nm}$, showing that the dynamics in thin films differs from that of the bulk, but the shift measured in peak maxima is about $10 \pm 1 \mathrm{~K}$ and in the relaxation onsets $26 \pm 2 \mathrm{~K}$.

The activation energy of $160 \mathrm{~kJ} / \mathrm{mol}$ calculated from the shift in the peak position due to the different eigenfrequency of the sample is almost $20 \%$ higher than the literature values. In order to get a more precise value, more measurements with different frequencies should be done and the difference in frequency should be at least one order of magnitude.

In Fig. 5.26 an overview of thicknesses determined by SEM micrography compared to those determined from the frequency shift is given. The fact that the film thicknesses calculated from the frequency shift are comparable to those measured by FIB/SEM analysis is a big advantage of the method for several reasons. The most important one is that one measurement gives both 


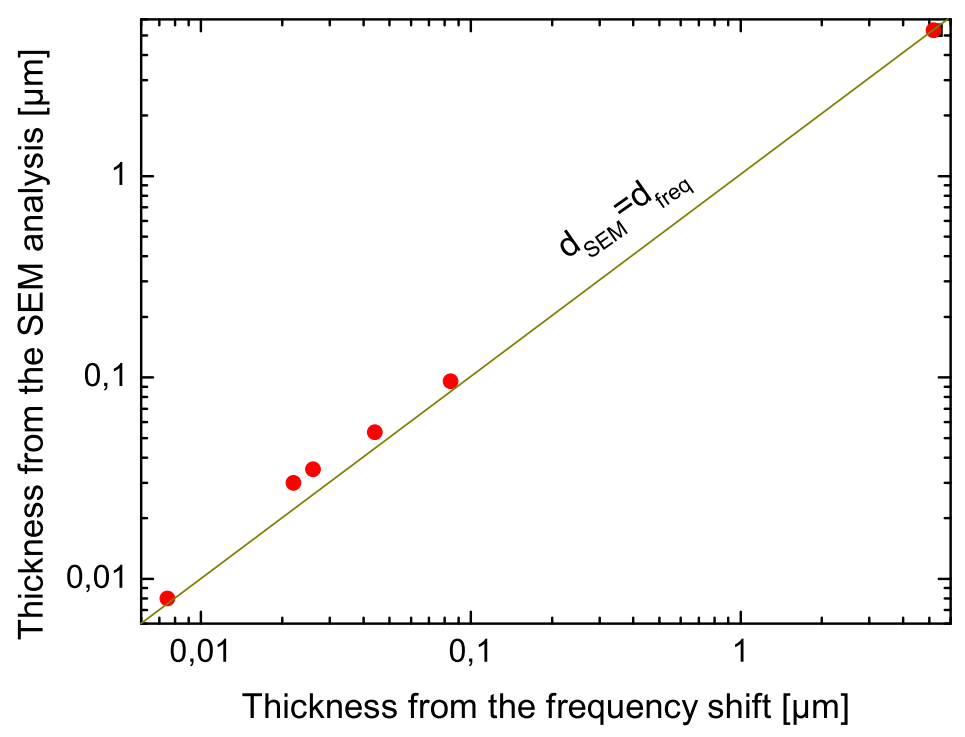

Figure 5.26: A comparison of film thicknesses determined from the frequency shift and from the SEM micrography. The film of $4.3 \mu \mathrm{m}$ thickness is the PVCN film from Section 6.4.

information, the relaxation spectrum and the film thickness, needed for the investigation of the effect of reduced dimensions on dynamics in thin films. The samples can be placed in the VRA immediately after applying the film and the chamber can be evacuated, preventing film oxidation. The annealing can be done within the apparatus in vacuum and in the dark, hence the solvent can evaporate faster, the relaxation of stresses within the film takes place and no moisture or UV light affects the film. The measuring of the film thickness by some other method directly on the composite cantilever would increase the risk of breaking the sample.

The vibrating reed as a resonant technique enables the nondestructive in situ measurement of the film thickness. The $\alpha$ relaxation of PS films broadens and the glass transition temperature shifts to the lower temperatures, but the effect on dynamics is not as dramatic as reported by Forrest and Dalnoki-Veress (2001) (Fig. 5.1).

Mechanical spectroscopy on thin films as one of the dynamic measurements gives information not only on the glass transition temperature, but on the whole relaxation spectrum. Within this work the depression in the relaxation temperatures as well as the broadening of the relaxation peak is measured, but stopped at a film thickness of $44 \mathrm{~nm}$. The experimental funding is attributed to the confinements at the film/vacuum and substrate/film 


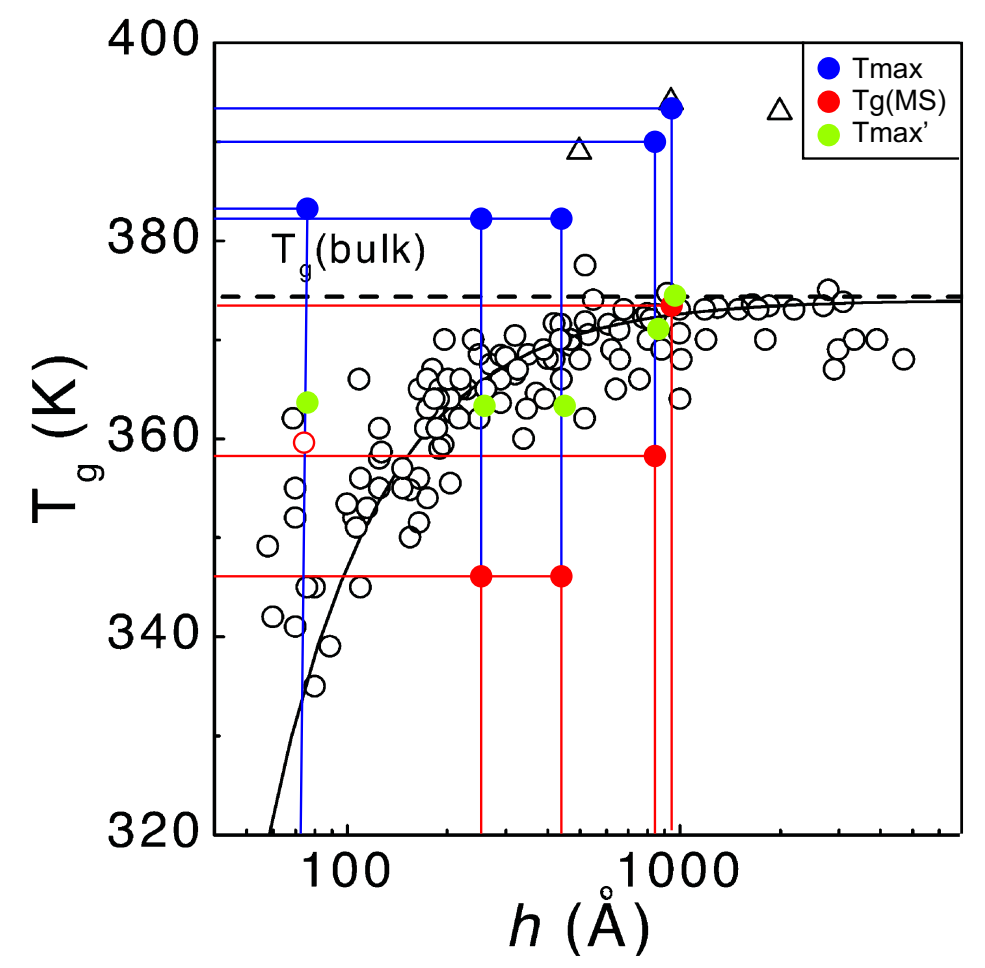

Figure 5.27: The results of this study on thin films are included in the overview diagram presented by Forrest (see Fig. 5.1). The red points present the relaxation onsets (in mechanical spectroscopy (MS) conventionally identified as $T_{g}$ ), the blue points present the relaxation maxima and the green points present the relaxation maxima shifted so that the temperature belonging to the $95 \mathrm{~nm}$ thin film fits to the glass transition temperature of PS. The shift is done only to make the comparison easier.

interfaces. 


\section{Chapter 6}

\section{Mechanical Spectroscopy of Poly(vinyl cinnamate) with Different Cross-linking Degree}

Cross-linking of polymers is very important for their application. Through cross-links, the dynamics of polymers is highly influenced leading to changes in materials properties.

In this chapter a contribution to revealing the problem of interrelation among the cross-linked structure, molecular dynamics and the material's properties is presented. The structure of polymers is modified in a controlled manner and the resulting changes in dynamics are observed. The effect of cross-linking on dynamics of polymer glasses below and above the glass transition temperature $\left(T_{g}\right)$ is studied with an emphasis to the problem of glass transition. The chemical bonds between molecules produced by crosslinking hinder molecular mobility and thus influence relaxation processes in the material. The mechanical response of a solid is related to the density of strong bonds; therefore the covalent bonds between the side chains make a material more rigid.

On the example of poly(vinyl cinnamate) (PVCN) by means of mechanical spectroscopy, dynamics of polymer molecules with increasing the crosslinking degree is studied. The polymer is cross-linked by irradiating it with ultraviolet (UV) light. The cross-linking is controlled by the irradiation time and its degree is observed by means of Fourier Transformed Infrared Spectroscopy (FTIR).

In Section 6.1 the mechanism of cross-linking is described by introducing the expected changes of the material behavior. Section 6.2 deals with the sensitivity of PVCN to the UV light which is revealed by a detailed FTIR analysis. Eventually, in Section 6.3 the mechanical spectra are presented 
with a careful analysis of the changes in the mechanical response by the increasing in cross-linking degree.

\subsection{Cross-linking}

In this section an introduction into the relation between the cross-linked structure and the mechanical response is given along with a brief description of the light induced cross-linking mechanism. Then basic information on the structure and properties of PVCN is presented and the expected behavior of its covalent bonds when UV irradiated is analyzed.

\subsubsection{Cross-linking and the mechanical response}

Cross-linking involves the formation of intermolecular connections through chemical bonds which occurs randomly. This process necessarily results in a three dimensional network and reduction of chain mobility.

As the cross-links density is increased, the restriction on molecular mobility becomes substantial and much higher energy will be required to induce segmental motions what leads to the increase in $T_{g}$. In the glassy region, $T<T_{g}$, independent of the cross-linking degree, the probability of conformation changes are very low and the mechanical response to small stresses (deformations) is basically anelastic. For $T>T_{g}$, the glass-to-rubbery region, the response is both anelastic and viscous. By cross-linking, the flow regions in the material become less pronounced.

The elastic modulus of a solid is related to the energy of a bond between neighboring atoms or molecules multiplied by the density of those bonds (Jones, 2003). The same force applied to a cross-linked and an uncross-linked polymer will impose higher deformation of the uncross-linked polymer. The increase in the number of covalent bonds in the polymer by cross-linking results in a mechanically harder and stiffer material.

Since cross-linked polymers are chemically tied together and solubility essentially involves the separation of solute molecules by solvent molecules, cross-linked polymers do not dissolve, but can only be swelled by liquids. The presence of cross-linking confers stability of polymers (Ebewele, 2000).

An uncross-linked polymer is a viscoelastic liquid in the sense that it does not possess any equilibrium compliance and exhibits viscous flow at sufficiently long times.

\subsubsection{Light induced cross-linking}

The exposure to radiation generally induces unwanted chemical reactions responsible for the degradation of polymer molecules and the modification of their properties. But light can also be utilized to promote some desired reactions, such as the polymerization of a monomer or the cross-linking of 
a polymer. The main interest of using light to induce a chemical reactions lies in the high initiation rate provided by intense illumination and a precise spatial and temporal control of reaction without a catalyst (Decker, 2002).

To induce a chemical reaction by light the energies absorbed by a molecule must be sufficient to break a covalent bond. In most chemical compounds the covalent bond energies correspond to wavelengths between $200 \mathrm{~nm}$ and $700 \mathrm{~nm}$. Thus the most effective radiation is of that type, which spans the visible and ultraviolet regions. Higher the number of electrons shared in a bond, the higher will be the bonding energy. For absorption to occur, two conditions have to be satisfied. First, for an initial molecular state with energy $E_{m}$ there must be a state $n$ of higher energy $E_{n}$ such that $h \nu=E_{n}-E_{m}$ and second, there must be a specific interaction between the electric component of the incident radiation and the chromophore which results in a change in the dipole moment of the molecule during the transition. Electronic transitions with high absorption probability are said to be 'allowed' and those with low absorption probability are said to be 'forbidden'.

After absorption of a photon a molecule exists in an excited state and because it is not in thermal equilibrium with its surroundings, it will have only a short lifetime since a number of chemical and physical processes can contribute to the deactivation of the excited molecule to the lower state (Guillet, 1985). In the following experiments the process that mostly contributes to the deactivation is the cross-linking as will be described in Chapters 6.1.3 and 6.2.2.

\subsubsection{Cross-linking of poly(vinyl cinnamate) (PVCN)}

Poly(vinyl cinnamate) (PVCN) is actually the copolymer of vinyl cinnamate and vinyl alcohol. Its glass transition temperature $T_{g}$ is about $77^{\circ} \mathrm{C}$. The polymer is stable up to $200^{\circ} \mathrm{C}$. Above this temperature the thermal degradation caused by cleavage of cinnamate unit from the main chain and crosslinking takes place (Kim and Park, 1998). This polymer is well known for its sensitivity to the UV radiation (Minsk et al., 1959), (Robertson et al., 1959). The UV light induces chemical reaction which leads to the crosslinked structure and a relatively soft polymer glass can thus be transformed into a hard and insoluble polymer.

PVCN is spatially isotropic (Yaroshchuk et al., 2002) and it undergoes a random cross-linking reaction by photoaddition between a UV excited cinnamoyl group of one polymer chain and that of an unexcited cinnamoyl group on another or the same chain, forming a cyclobutyne ring as schematically depicted in Fig. 6.1 (Coleman et al., 1998). The carbonyl group of an ester will not be UV excited, because according to (Guillet, 1985), a carbonyl group of an ester will be excited while irradiated by short-wavelength radiation, usually $254 \mathrm{~nm}$. The electrons in single bonds are usually held too tightly to be excited by near ultraviolet radiation. Hence only $\mathrm{C}=\mathrm{C}$ 


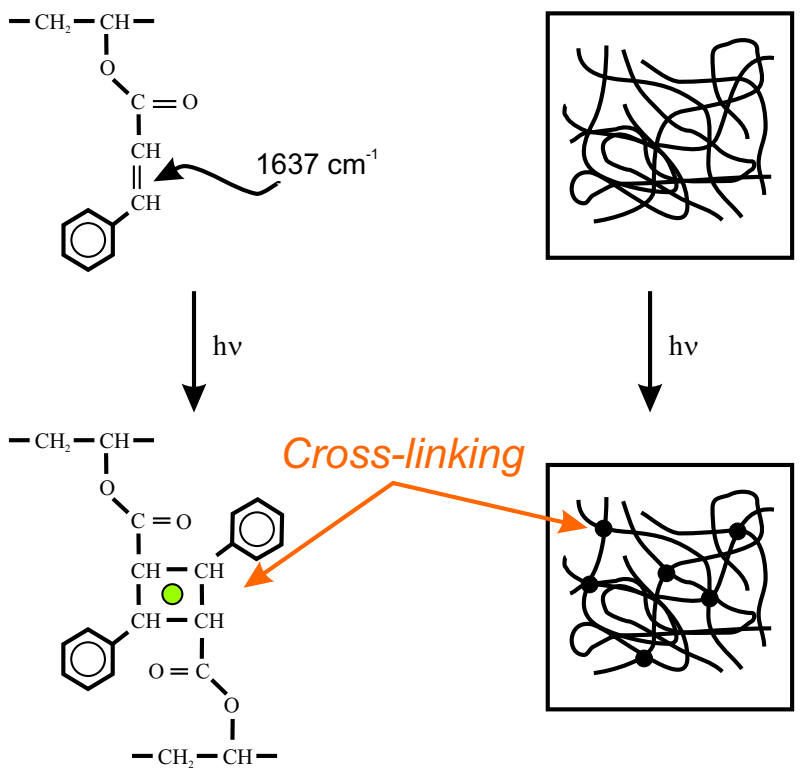

Figure 6.1: Cross-linking of PVCN. Photoaddition between a UV excited cinnamoyl group with that of an unexcited cinnamoyl group.

double bond will cross-link when PVCN is irradiated by UV light.

\subsection{Cross-linking degree assessment by Fourier Trans- formed Infrared Spectroscopy (FTIR)}

The term "infrared" covers the range of the electromagnetic spectrum between 0.78 and $1000 \mu \mathrm{m}$. In the context of infrared spectroscopy, wavelength $\lambda$, is measured in "wavenumbers" $\nu$, which have the units $\left[\mathrm{cm}^{-1}\right]$ and $\nu=1 / \lambda$. The region that is the most useful lies between $4000-500$ $\mathrm{cm}^{-1}$.

Unlike UV, IR radiation does not have enough energy to induce electronic transitions as with UV. Absorption of IR is restricted to compounds with small energy differences in the possible vibrational and rotational states. For a molecule to absorb IR, the vibrations or rotations within a molecule must cause a change in the dipole moment of the molecule. The alternating electrical field of the radiation interacts with fluctuations in the dipole moment of the molecule. If the frequency of the radiation matches the vibrational frequency of the molecule then radiation will be absorbed, causing a change in the amplitude of molecular vibration.

Since each bond absorbs different wavelengths, the cross-linking can be nicely studied by infrared (IR) spectroscopy. In the following experiment the disappearance of the IR band characteristic of the $\mathrm{C}=\mathrm{C}$ double bond is monitored upon UV exposure. 
The FTIR spectra can be presented either as absorbance or as transmittance of energy.

\subsubsection{Preparation of samples for FTIR}

For this experiment films of several microns are prepared by spin-coating the same polymer solution on silicon plates. The samples are then dried at the temperature slightly above $T_{g}$ for 2 hours in order to evaporate the solvent (tetrahydrofuran) remained after spin-coating. The surface of the substrate used in the experiment has to be reflective and the polymer film transparent, so that the incident beam can be transmitted through the film and reflected onto the detector. The spectra show the energy loss of the incident beam, or the transmittance through the film to the detector.

After drying and relaxing the film is photo-cross-linked using a 200 W mercury-xenon lamp (Hamamatsu, L8333) with the intensity of 3500 $\mathrm{mW} / \mathrm{cm}^{2}$ at $365 \mathrm{~nm}$. The sample holder along with the sample is placed at a distance of $18 \mathrm{~cm}$ from the source. The degree of cross-linking is controlled by the photo-irradiation time.

The exposure intervals are between 2 and 20 min and the overall exposure time is $60 \mathrm{~min}$. The irradiation is carried out in air at room temperature. The temperature increase in the sample is $2^{\circ} \mathrm{C}$ for 2 min exposure and $4^{\circ} \mathrm{C}$ for the 10 min exposure.

\subsubsection{Infrared spectra depending on the UV irradiation time}

The FTIR is done in cooperation with the Pulsed Laser Deposition (PLD) group at the Institute for materials physics in Göttingen (Süske, 2005) by means of the spectrometer Bruker Tensor 35. The angle of incidence is $5^{\circ}$ and the resolution $4 \mathrm{~cm}^{-1}$. The transmittance is the ratio of a film spectrum and a spectrum of a pure Si substrate.

The overview (500 to $4000 \mathrm{~cm}^{-1}$ ) fingerprint spectrum of the PVCN before UV exposure is shown in Fig. 6.2. Between 3250 and $3600 \mathrm{~cm}^{-1}$ is a broad band attributed to the $\mathrm{O}-\mathrm{H}$ stretching vibrations of the poly(vinyl alcohol) proving that the polymer is a copolymer of vinyl cinnamate and vinyl alcohol. A relatively sharp IR bands, characteristic of an essentially conformationally insensitive phenyl ring are evident between 3100 and 3000 $\mathrm{cm}^{-1}$ (C-H stretch), at $\approx 1580,1500$ and $1450 \mathrm{~cm}^{-1}$ (ring stretches), $\approx 770$, 720 and $680 \mathrm{~cm}^{-1}$ (ring modes and $\mathrm{C}-\mathrm{H}$ wag) and between 1780 and 2000 $\mathrm{cm}^{-1}$ (the so-called summation bands) (Coleman et al., 1998).

However, for this study the band characteristic of the $\mathrm{C}=\mathrm{C}$ at 1637 $\mathrm{cm}^{-1}$ and that of the $\mathrm{C}=\mathrm{O}$ between 1700 and $1750 \mathrm{~cm}^{-1}$ are the most important. These bands can be seen in the expanded spectrum from 1500 to $1900 \mathrm{~cm}^{-1}$ (Fig. 6.3). As depicted in Fig. 6.1 the number of $\mathrm{C}=\mathrm{C}$ double bonds decreases during cross-linking and the change in the attributed band 


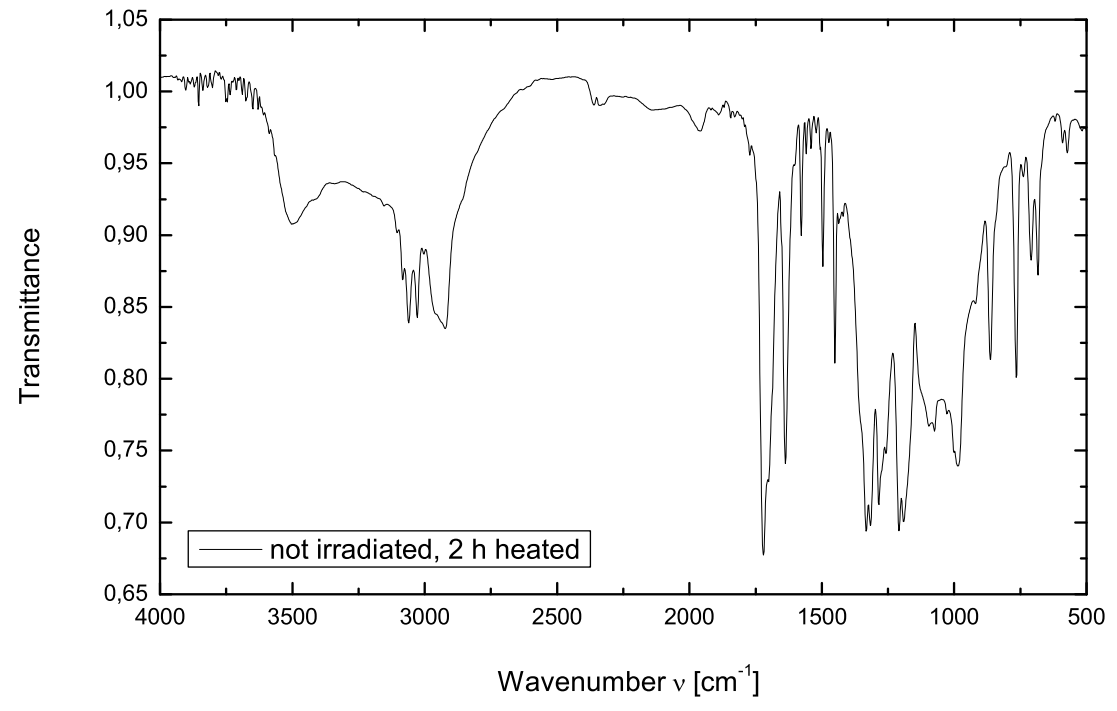

Figure 6.2: FTIR of PVCN, an overview spectrum of a polymer film before irradiation.

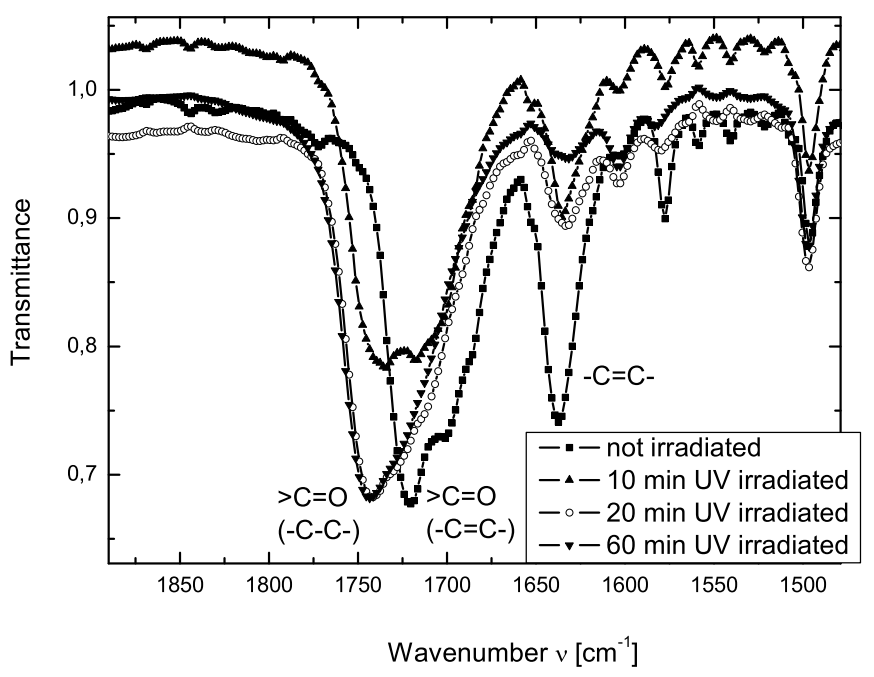

Figure 6.3: A detail of FTIR spectra of PVCN depending on the UV exposure time with the bands that are changed by UV light. 
with the time of exposure to the UV light can be observed in the IR spectrum and is the simplest way to estimate the cross-linking degree. On the other hand the number of carbonyl or phenolic hydroxyl functional groups remains constant, however before the exposure all the carbonyl groups are of the unsaturated ester type (i.e. $\mathrm{C}=\mathrm{C}-\mathrm{CO}-\mathrm{C}$ ) whereas after exposure to $\mathrm{UV}$ radiation those involved in the cross-linking reactions are now saturated esters. The shift of the $\mathrm{C}=\mathrm{O}$ band of the unsaturated ester type to that of the unsaturated type also provides the information on the cross-linking degree (Coleman et al., 1998).

The most dramatic changes in the infrared spectrum occur in the first 10 minutes. The bands of the $\mathrm{C}=\mathrm{C}$ double bond and that of the carbonyl group of the unsaturated ester decrease with exposure time. Concurrently, a new band, attributed to a saturated ester carbonyl stretching band occurs and its intensity increases. According to (Matsuguchi et al., 2000) the thinner the film is, the higher the degree of cross-linking will be for the same photoirradiation time. They also report that PVCN will cross-link nearly $100 \%$ after 48 hours irradiation. The absorption depth is higher then the film thicknesses in the experiment, though the maximal thickness is not tested.

\section{Kinetics of cross-linking}

The kinetics of the cross-linking is shown in Fig. 6.4. In order to avoid the experimental inaccuracy, the $\mathrm{C}=\mathrm{C}$ band is by integration normed to the stretching $\mathrm{C}-\mathrm{H}$ band. Independent of the exposure intervals the kinetics seems to be the same and the results are reproducible.

However, it is interesting to notice that the sample that is irradiated in 2 min intervals did not show any change in the spectrum for the first three intervals and afterward the decrease of the $\mathrm{C}=\mathrm{C}$ band is the same as that of the samples irradiated for longer intervals. This effect is more obvious in the logarithmic time scale. A possible explanation would be that the polymer contains something that inhibits the cross-linking by terminating the radicals and when it is consumed, the cross-linking can start. This process is called "self cleaning" (Buback, 2005). This would explain why the decay of the $\mathrm{C}=\mathrm{C}$ band is independent of the exposure intervals.

For the reaction to take place an unsaturated cross-linker has to come within a short reaction radius $r_{x}$ of an excited cross-linker. This process is diffusion dependent. Since the glassy polymer at room temperature has a rather high activation energy for the inter diffusion there must be either a critical concentration of excited cross-links in the film which would be able to search for the unsaturated couple in the nearest neighborhood or some local movements. After the reaction started the number of cross-links decays with the logarithmic dependence on irradiation time. At the final state some cross-linkers will be unable to reach an unsaturated cross-linker. This happens because the cross-linking itself leads to a spacial confinement 

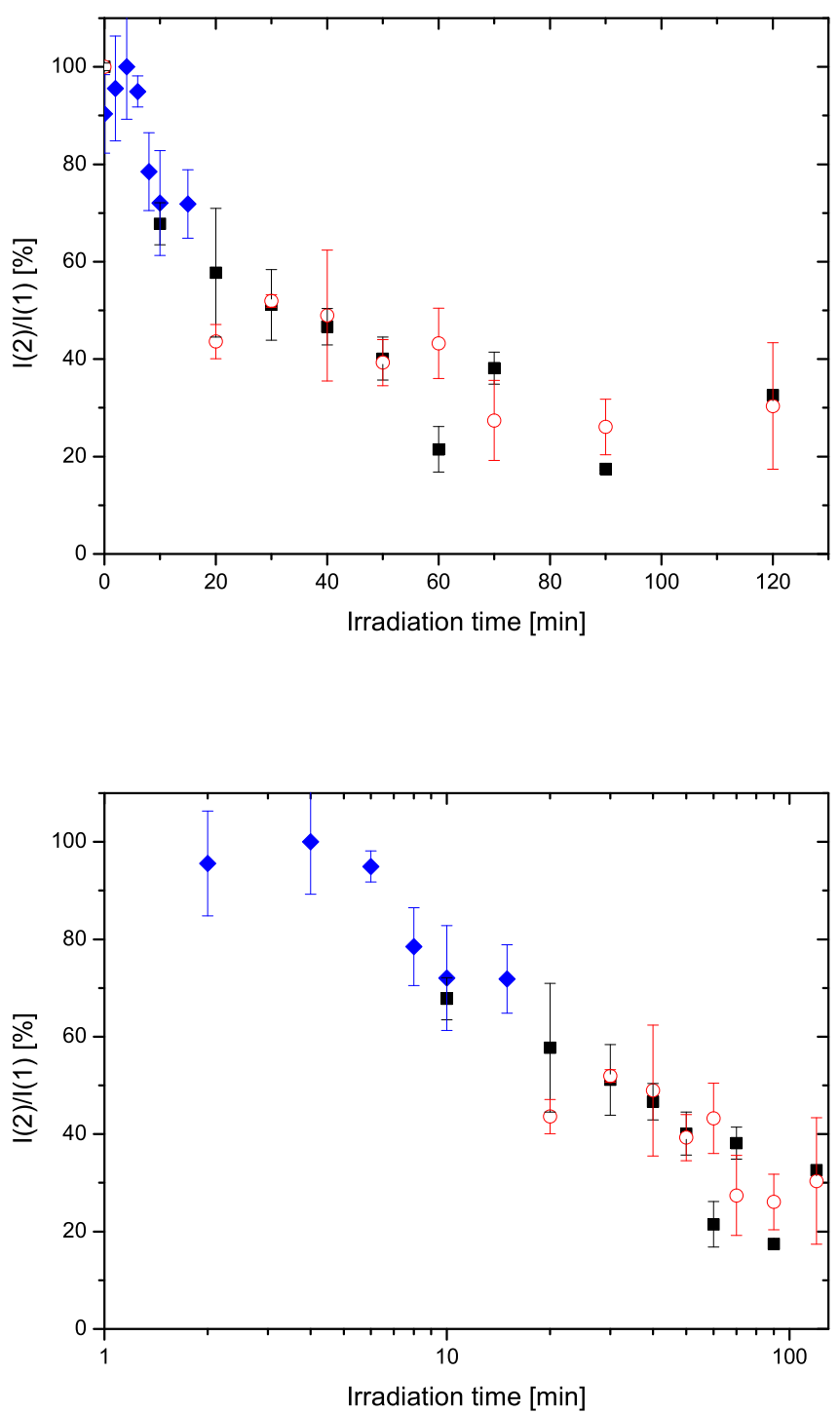

Figure 6.4: The intensity decrease in the $\mathrm{C}=\mathrm{C}$ band with the irradiation time. The intensity of the $\mathrm{C}=\mathrm{C}$ band is normalized to that of the $-\mathrm{CH}$ band. Three samples are analyzed, each is presented by a different symbol. In the upper figure the intensity ration is plotted versus time and in the lower figure versus the logarithm of time. 
of the chains. They can only explore a rather small volume in order to look for linking partners (Grest et al., 1992). This leads naturally to some unsaturated links.

\subsection{Mechanical spectra depending on cross-linking degree}

\subsubsection{Film preparation for VRA measurements}

For these experiments the mechanically polished silicon cantilevers, Fig. 4.3 are used. A polymer film is applied on the upper surface.

Poly(vinyl cinnamate) (PVCN) of weight averaged molecular weight of ca. 200000 (GPC) is purchased from Aldrich Chemical Company, Inc. The material has the glass transition temperature of $77^{\circ} \mathrm{C}$ and is stable up to $200^{\circ} \mathrm{C}$ (see Chapter 6.1.3). Without further material purification $2.5 \%$ solution of PVCN in tetrahydrofuran (THF) is made. In order to see a pronounced effect of cross-linking a rather thick film with bulk properties is made by spreading the solution on the Si substrate evenly with a syringe and needle. After a few minutes when the majority of the solvent evaporated, the sample is mounted in the sample holder of the vibrating reed apparatus within a chamber which is evacuated afterwards. The film is transparent for the laser beam, so the reflection from the polished Si surface to the PSD could be recorded. The chamber is evacuated and heated to $90^{\circ} \mathrm{C}$ (above the glass transition temperature) to evaporate the rest of the solvent and to relax the residual stresses in the film. The temperature is kept constant for at least 3.5 hours at $2 \cdot 10^{-6}$ mbar in the dark. The sample remains in the apparatus for 4 days at the room temperature at a pressure of $10^{-6} \mathrm{mbar}$.

There is not any noticeable difference between spin-coated films and those applied by a syringe, both by mechanical spectroscopy and by FTIR. The only difference is that one has to have rather concentrated solution to spin-coat a film of the same thickness as that of the film applied by a syringe. Also Forrest and Sharp (2003) analyzed the influence of the film preparation on the glass transition temperature of PS films and reported no difference.

In order to cross-link the film, the sample is taken out of the vacuum chamber together with the sample holder and is placed $18 \mathrm{~cm}$ from the UV source. After irradiation, the sample holder is mounted back into the VRA and evacuated. The UV exposure intervals are 10, 30 and 120 minutes.

\subsubsection{Mechanical spectra}

Fig. 6.5 depicts mechanical spectra of the PVCN film on the Si substrate with an increase in cross-linking degree. The quality factor $Q^{-1}$ of the pure reed is between $2 \cdot 10^{-5}$ and $4 \cdot 10^{-5}$ depending on temperature. The $Q^{-1}$ value of the composite (substrate with a polymer film on it) is about 


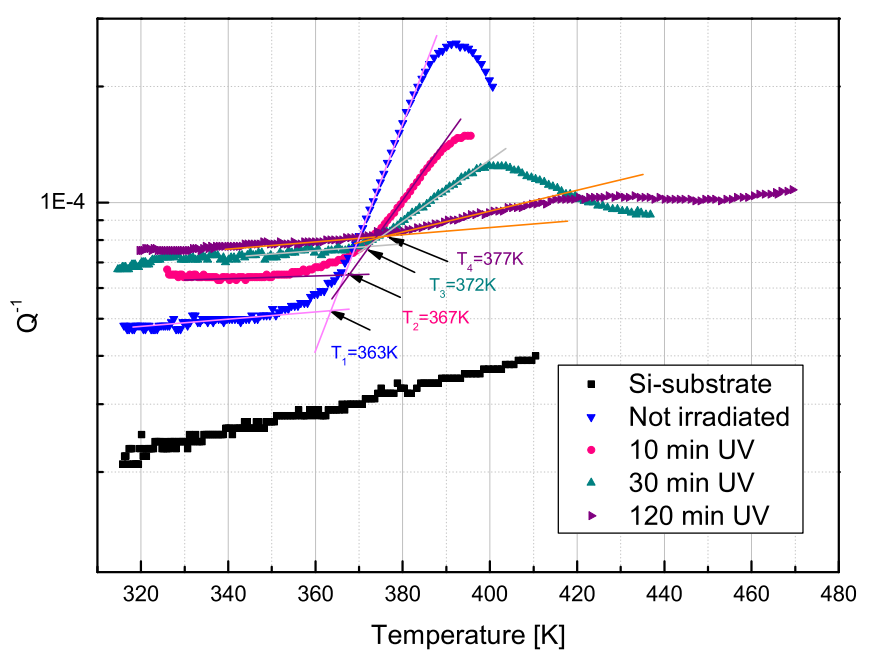

Figure 6.5: Mechanical spectra of PVCN depending on the UV exposure time. The spectra are of the composite, a Si-substrate and a polymer film. For further analysis the spectrum of the pure substrate has to be subtracted.

$5 \cdot 10^{-5}$ for the glassy state of PVCN. During the $\alpha$ relaxation it increased to the maximal value of about $5 \cdot 10^{-4}$. Dramatic changes in the spectrum occurred after the sample is exposed for 10 minutes to the UV irradiation. In the glassy state the $Q^{-1}$ value increases, whereas the relaxation strength decreases. The relaxation start or the glass transition temperature $T_{g}$ (Fig. 6.6) and the peak temperature (Fig. 6.7) shifts to higher values. With further irradiation this tendency continues consistently. After 120 minutes the relaxation almost vanishes.

In order to see the relaxation of the polymer without the substrate, the damping of the substrate has to be subtracted. The accurate values of $\Delta Q^{-1}$ follow from Eq. 3.51, which requires known dependence of both elastic moduli, Si and PVCN, on temperature. Here $\Delta Q^{-1}$ is calculated as the difference of $Q^{-1}$ factors of the composite and of the substrate; $\Delta Q^{-1}=$ $Q_{c}^{-1}-Q_{s}^{-1}$. The relaxation peaks are symmetric and fit nicely to the Gauss curve (Fig. 6.7). This makes the comparison much simpler. The Gauss fits of the subtracted spectra shifted to the 0 base line (Fig. 6.8) show how pronounced the changes are in the relaxation strength with the irradiation time. From the curves normalized to the same amplitude (Fig. 6.9) one can notice that the peaks are not only shifted, but also the relaxation became broader. In order to compare the width the peaks are set to the same temperature (Fig. 6.10). After 10 min irradiation the majority of changes happened, both in the relaxation strength reduction and in width. 


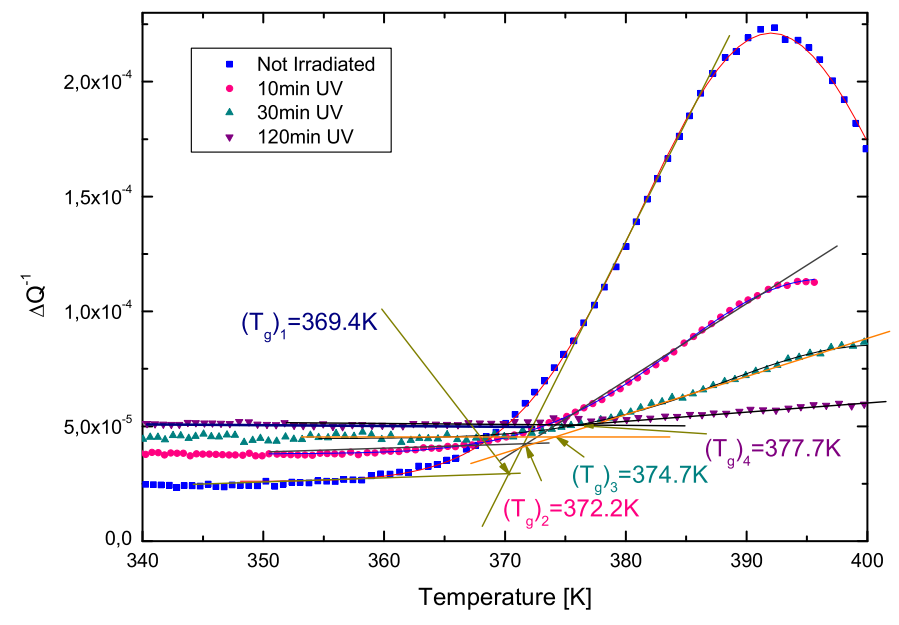

Figure 6.6: The glass transition temperatures, $T_{g}$, as the intersect of tangents. $T_{g}$ increases with the UV exposure time. The damping of the substrate is subtracted.

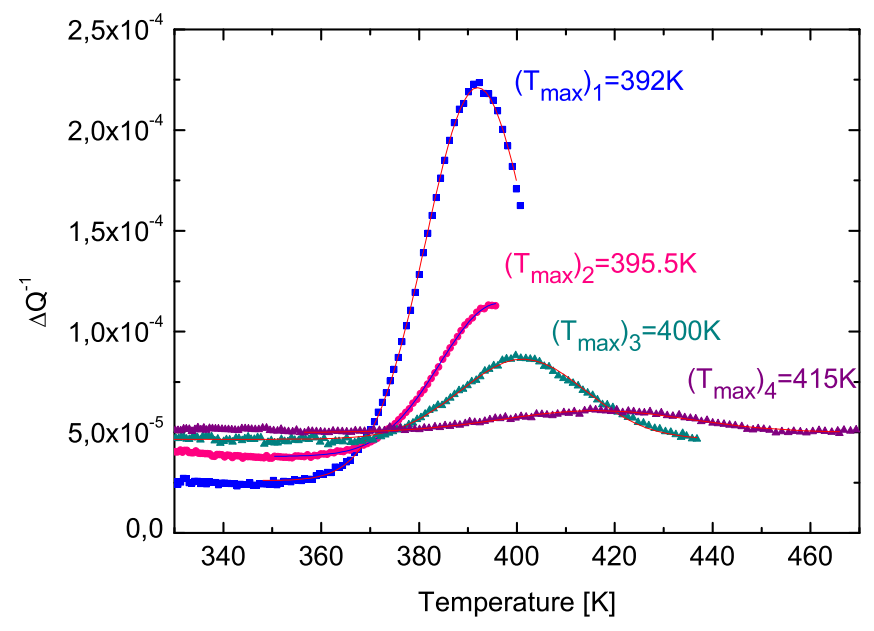

Figure 6.7: Mechanical spectra of PVCN for various UV exposure times, after subtraction of substrate values. The peak maxima shift due to cross-linking. The line graphs are of the Gauss fit. 


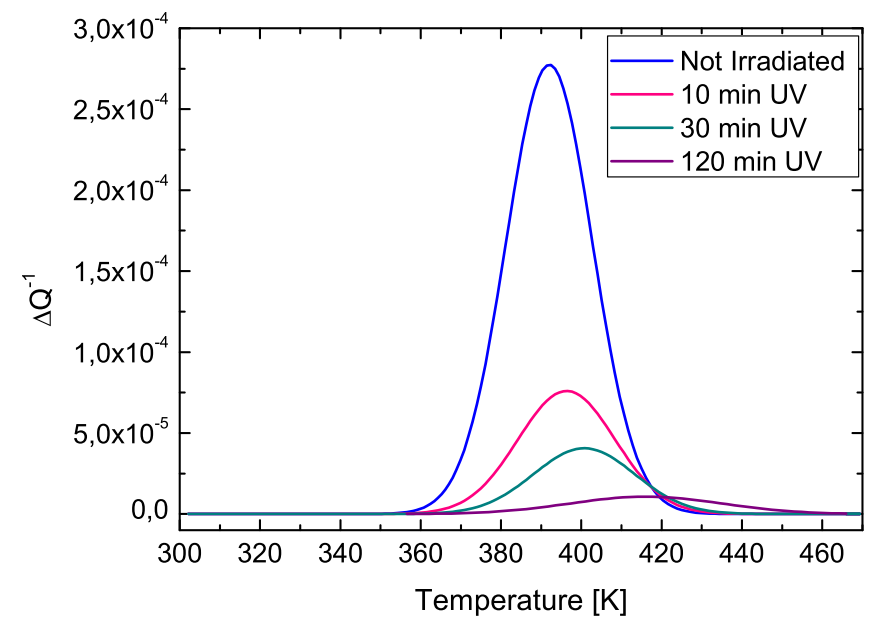

Figure 6.8: Gauss fits of the mechanical spectra. Curves are shifted to the 0 base line in order to compare the absolute decrease in the peak height caused by the cross-linked structure.

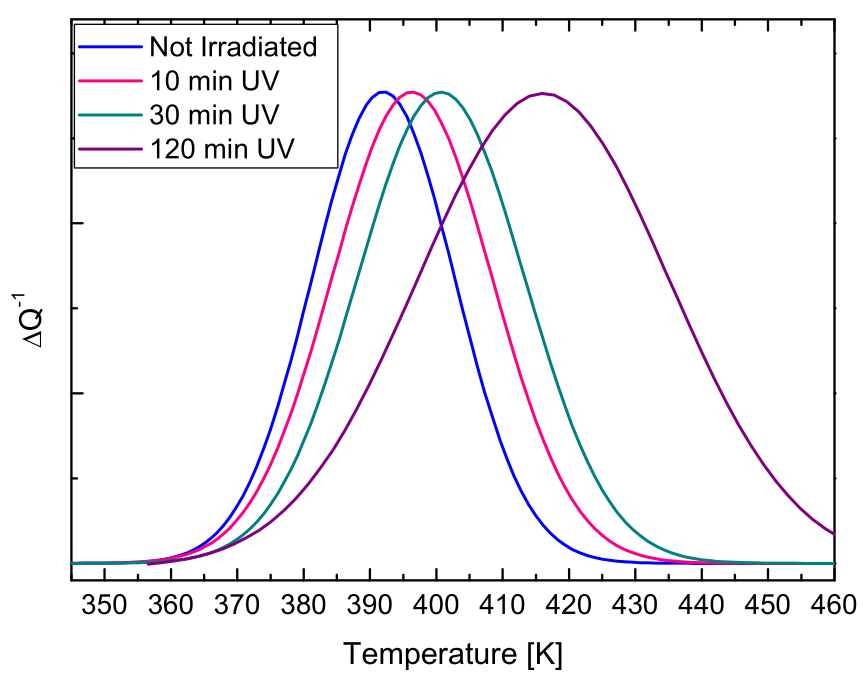

Figure 6.9: Gauss fits of mechanical spectra normed to the same amplitude in order to compare the peak shift caused by cross-linking. 


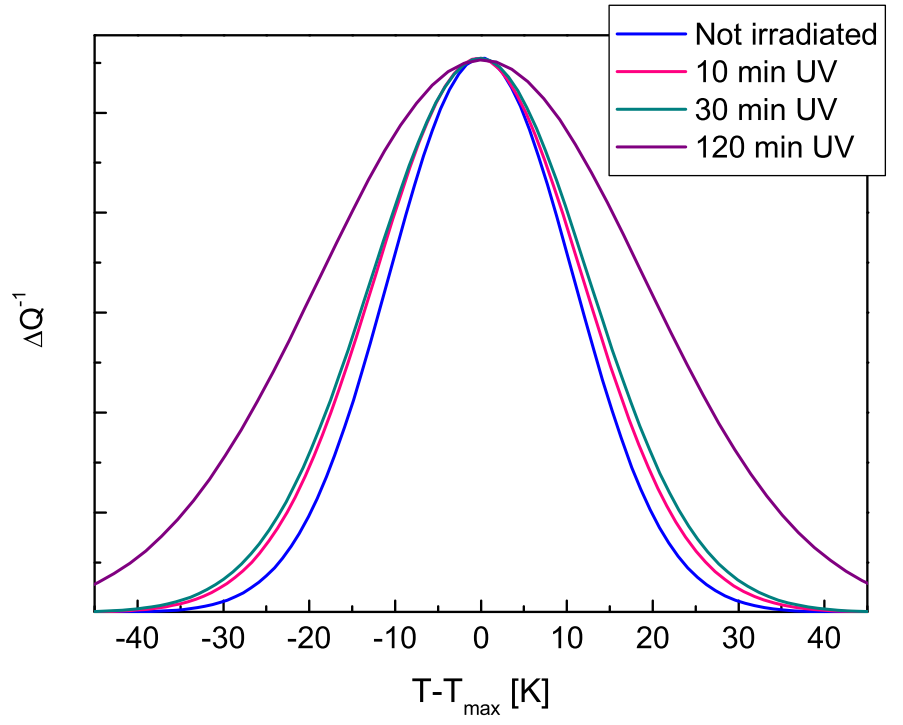

Figure 6.10: Gauss fits normalized to the same amplitude and shifted to the same peak position in order to compare the width of the relaxation.

\subsubsection{Eigen frequency influenced by cross-linking}

The eigen frequency of the sample depends on the mass, the elastic modulus and on the sample dimensions (Eq. 3.18). At $300 \mathrm{~K}$ for the pure Si substrate used in this experiment it is equal to $639.5 \mathrm{~Hz}$ (Fig. 6.11). Since the elastic modulus is temperature dependent, the frequency also changes with temperature. Here the slope of the frequency depending on temperature is $-2.04 \cdot 10^{-2} \mathrm{~Hz} / \mathrm{K}$ and $f \propto \sqrt{E}$ (Eq. 3.18).

After the film is applied the mass of the sample increases and thus the eigen frequency decreases by $\Delta f=-5.2 \mathrm{~Hz}$. From this shift one can calculate the film thickness (Eq. 3.20). The thickness of the substrate calculated from the eigen frequency is $d_{s}=105 \mu \mathrm{m}$, the density of $\mathrm{Si}$ is $\rho_{s}=2.33$ $\mathrm{g} / \mathrm{cm}^{3}$ and the elastic modulus of $\operatorname{Si}(100)$ is $E_{s}=163 \cdot 10^{9} \mathrm{~Pa}$. The polymer does not sink in the water (the water density is $\approx 1 \mathrm{~g} / \mathrm{cm}^{3}$ ), and it hardly sinks in the toluene (the toluene density is $0.865 \mathrm{~g} / \mathrm{cm}^{3}$ ), so the estimated polymer density is assumed to be $\rho_{f}=0.9 \mathrm{~g} / \mathrm{cm}^{3}$. The elastic modulus of non-cross-linked polymers is about $E_{f}=3 \cdot 10^{9} \mathrm{~Pa}$ (Van Krevelen, 1990). With these values the thickness is $d_{f}=5.18 \mu \mathrm{m}$.

The frequency of the UV exposed sample increases consistently with the increase in the cross-linking degree (Fig. 6.11 and Fig. 6.12). Since the mass remains constant, the effect is caused by the increase in the elastic modulus of the cross-linked structure. The frequency of the $120 \mathrm{~min}$ irradiated sample 


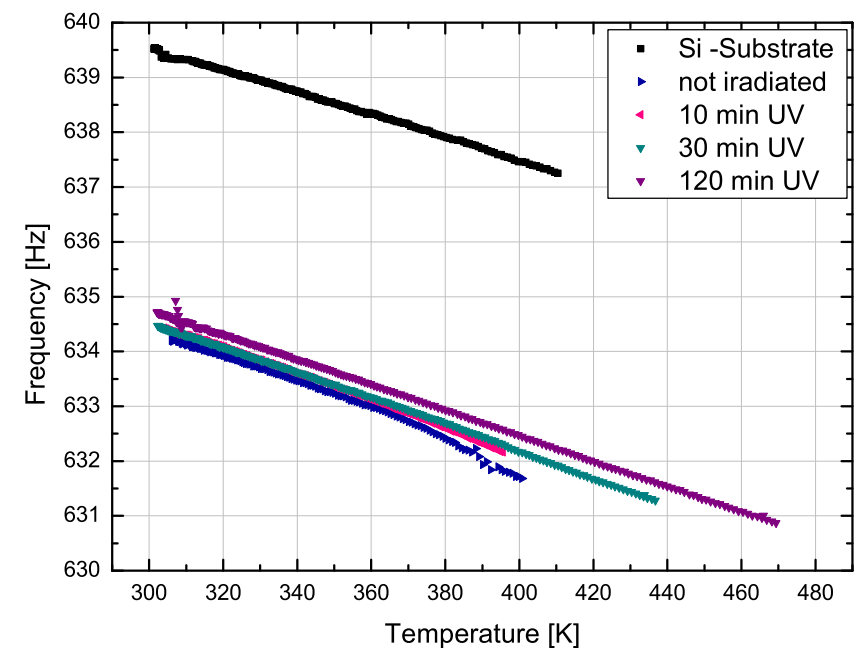

Figure 6.11: Eigen frequencies of the sample. The frequency of the substrate is compared to that of the composite, the substrate with the film applied on it. Then the film is irradiated with the UV light resulting in the increased elastic modulus s concomitant increase of eigen frequency.

compared to that of the not irradiated one is increased for $\Delta \Delta f=0.4 \mathrm{~Hz}$ at $300 \mathrm{~K}$. There is a slight increase in the absolute value of slope of the curve describing the eigen frequency dependence on temperature of the composite $(-0.023)$ comparing to the pure Si reed $(-0.020)$ proving that the modulus of the cross-linked polymer is more temperature sensitive than that of $\operatorname{Si}(100)$. By uncross-linked polymers the slope of the elastic modulus depending on temperature is the same as that of the cross-linked below the $T_{g}$ where the viscous flow starts.

From the frequency shift $\Delta \Delta f$ of the film which is irradiated for $120 \mathrm{~min}$ and has about $70 \%$ of the structure cross-linked, the elastic modulus can be calculated. From (Eq. 3.23) $E_{f x}=4.4 \cdot 10^{9}$ Pa follows. The elastic modulus of cross-linked PVCN is about $50 \%$ higher than that of the uncross-linked structure. This is in agreement with (Van Krevelen, 1990). Introducing new covalent bonds in the structure results in the higher overall energy of bonding and thus the higher elastic modulus (see Section 6.1.1). The increase of the elastic modulus of the film and the frequency shift depending on the amount of cross-links generated by the UV exposure and measured by the FTIR is depicted in Fig. 6.12.

Since the mass of the sample and thus the product of the density and thickness before and after cross-linking remains constant, $\rho_{f} d_{f}=\rho_{f x} d_{f x}$, a slight possible change in the density caused by the ordered structure which 


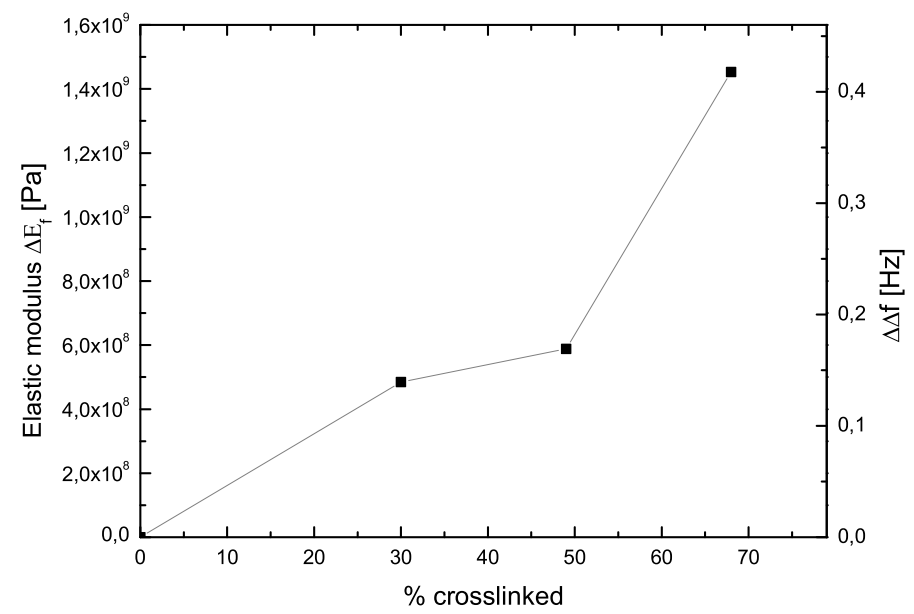

Figure 6.12: The elastic modulus of the cross-linked structure increases by about $50 \%$ after about $70 \%$ of $\mathrm{C}=\mathrm{C}$ double bonds cross-linked. This causes the change in the eigen frequency of the composite sample. The line is drawn to guide the eye.

would result in the change in the film thickness was not tested.

The frequency dependence on temperature is linear up to the glass transition temperature where the curve undergoes inflection. The inflection of the curve decreases with the decrease in the relaxation strength. The more pronounced inflection of the uncross-linked film means higher $\Delta \Delta f$, so from Eq. 3.23 it is obvious that the elastic modulus above $T_{g}$ grows faster with cross-linking than in the glassy state, because the viscous flow of the $\alpha$ relaxation is strongly affected by the joints between molecules.

\subsubsection{Discussion on mechanical spectroscopy}

The mechanical spectra of PVCN with different degree of cross-linking give us information on how the cross-linked structure influences the glass transition and how do mechanical properties change depending on temperature and phase.

The obvious observation in the spectra is that the $\alpha$ relaxation peak diminishes with cross-linking. The peak becomes broader and shifts to higher temperatures. The quality factor of the composite (the film on the substrate) $Q^{-1}$ at $T<T_{g}$ increases with the UV exposure time. This change $\Delta Q^{-1}$ is basically caused by the increase of the modulus $E_{f}$ with increasing cross-link density, but also some other effects are observed. 


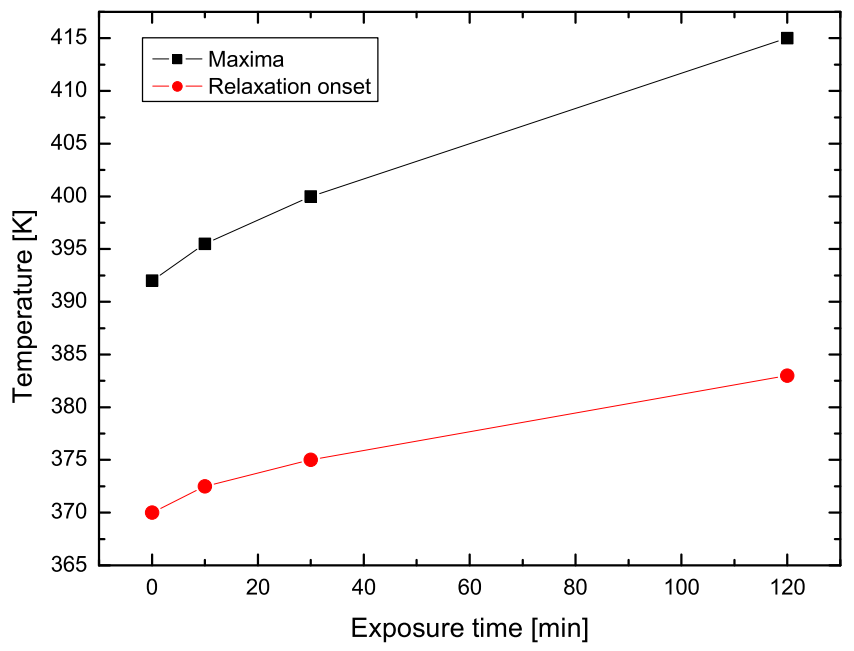

Figure 6.13: Increase in the glass transition temperature with the exposure time. The peak temperatures increase faster then those of the glass transition temperatures, because the relaxation becomes broader. The lines are drawn to guide the eye.

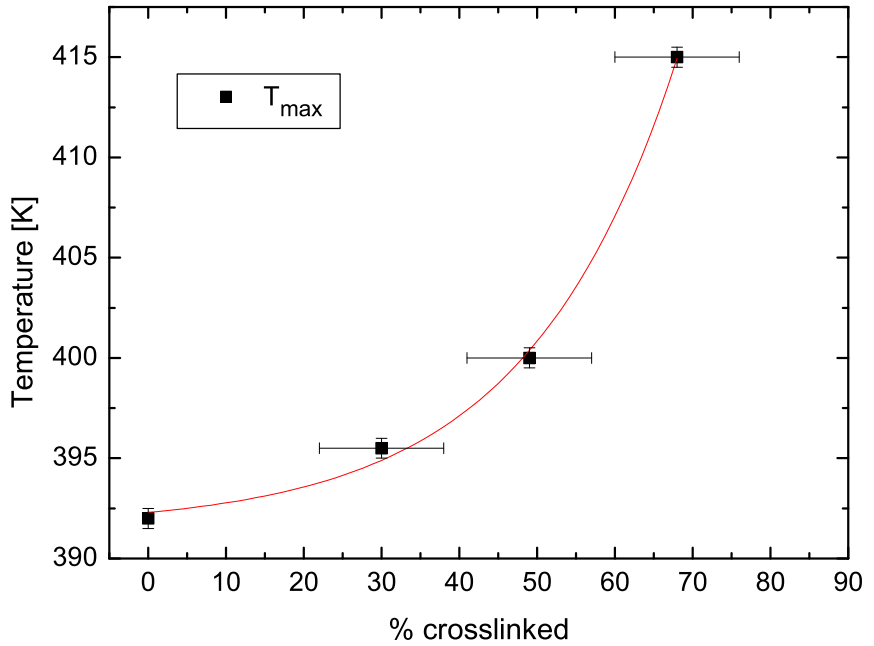

Figure 6.14: The temperatures of the maxima in the mechanical spectra depending on the cross-linking degree. The peak temperature diverges when the fraction of cross-links is about $75 \%$. (The curve is drawn to guide the eye.) 


\section{Glass transition}

Since the $\alpha$ relaxation of polymers is caused by movements of chains and segments the hindrance through the cross-links leads to the lower internal friction during the relaxation process. Here only the standard WLF jumps take place, because according to (de Gennes, 2000) for bulk samples the sliding process is completely inefficient.

A polymer with a backbone that exhibits higher flexibility will have a lower $T_{g}$. This is because the activation energy for conformational changes is lower. Therefore, conformational changes can take place at lower temperatures. Contrary, in order to excite the cross-linked molecular net of high molecular weight, higher energy (or temperature) is required, so the relaxation takes place at higher temperatures and the average relaxation time becomes longer. The new structure entails heterogeneity of the chain environment and the length of the units participating in the relaxation process. This leads to the large distribution of the relaxation times and thus implies a broadening of the relaxation, as shown in Fig. 6.10. The dependence of $T_{g}$ and peak maxima on the exposure time is depicted in Fig. 6.13.

After the sample is exposed for $120 \mathrm{~min}$ to the UV light the relaxation almost vanished. The total extinction of the peak would mean that the viscous flow is totally hindered by cross-links. As confirmed by the IR spectra (Fig. 6.3) the structure is not completely cross-linked. The reason for that and the mechanism of cross-linking is described in Section 6.2.2. How the peak position from the mechanical spectra shifts depending on the amount of cross-links is depicted in Fig. 6.14. The values are taken from the IR spectra (Fig. 6.4) and the mechanical spectra (Fig. 6.7). Obviously the peak temperature diverges for the fraction of about $75-80 \%$. This means that the material becomes a solid. This is in agreement with the IR spectra showing saturation in cross-linking for the same fraction.

The decrease of the peak can additionally be explained with the help of Eq. 3.51. Cross-linking enhances the elastic modulus (see Sections 6.3.3 and 6.1.1). The drop in the quality factor of the sample $Q^{-1}$ can only be reduced by the reduction in the quality factor of the film $Q_{f}^{-1}$. In the glassto-rubbery $\alpha$ transition the cross-linking reduces viscous flow and thus the losses in the material. This leads to the lower internal friction of the polymer film resulting in lower vibration damping.

\section{Glassy state}

In the glassy state, however, the quality factor of the composite increases. The cause of the quality factor enhancement by the UV exposed sample in the glassy state is rather complex. In Section 6.3.3 the change in the elastic moduli is calculated. For the uncross-linked PVCN it is $E_{f}=3 \cdot 10^{9} \mathrm{~Pa}$ and for the cross-linked $E_{f x}=4.4 \cdot 10^{9} \mathrm{~Pa}$. From Eq. 3.51 one can calculate the 
shift in the quality factor of the film $Q_{f}^{-1}$ relative to the substrate before and after cross-linking. The shift in the $Q^{-1}$ factor at $300 \mathrm{~K}$ before cross-linking is $\Delta Q^{-1}=2.5 \cdot 10^{-5}$ and after cross-linking $\left(\Delta Q^{-1}\right)_{(x)}=5.2 \cdot 10^{-5}$ (Fig. 6.5). From Eq. 3.51 follows $Q_{f}^{-1} / Q_{f x}^{-1}=0.73$. This means that for $T>T_{g}$ internal friction decreases with cross-linking and for $T<T_{g}$ it increases.

The internal friction can be increased by increasing a number of relaxation centers, the relaxation strength (the initial and final strain) or by decreasing the activation energy. The activation energy of a cross-linked structure increases; therefore the reason for this behavior is either in the relaxation centers enrichment or the increase in the relaxation strength. Due to the cross-linking the reduction in the distance between cross-links leads to the increase in the number of relaxation centers. Even in the transition are one can notice that the "base line" of the spectra shifts to the higher $Q^{-1}$ values, though the relaxation strength decreases strongly due to the hindernis to the viscous flow (see Fig 6.7).

From mechanical spectra one gets interesting information about the materials behavior at some temperature range that can be of interest for an industrial application. By simply controlling the exposure time, one can tailor the material properties needed. The changes in the properties below and above $T_{g}$ are not the same and one has to be aware that $T_{g}$ is frequency dependent. The change in the damping, the increase in elastic modulus and the reduction of the viscous behavior leading to incapability to melt combined with the resistivity of the polymer to solvents may have a large application field as coatings or isolation.

\subsection{SEM Analysis}

In Fig. 6.15 the cross section of the reed with the cross-linked film on it used for these measurements can be seen. The sample was placed into liquid nitrogen, taken out and broken within a second. Since silicon is a good heat conductor the time is enough to increase the temperature enough to see the ductile behavior of the polymer. This can be seen on the right hand side of Fig. 6.15. Breaking the sample inside the liquid nitrogen would prevent the ductile fracture. The film surface is rather smooth and uniform on the whole cross section with the exception at the edges where its thickness decreases (Fig. 6.15).

SEM analysis shows that the film thickness is $5.3 \mu \mathrm{m}$. The film thickness calculated from the eigen frequency shift (see Chapter 6.3.2) is $5.18 \mu \mathrm{m}$ which is in very good agreement with the SEM analysis.

The difference of about $3 \%$ is mainly due to the curved surface at the edges (Fig. 6.15), where the film thickness decreases. The thickness calculated from the frequency shift is an average.

For mechanical spectroscopy of films with bulk properties the film thick- 


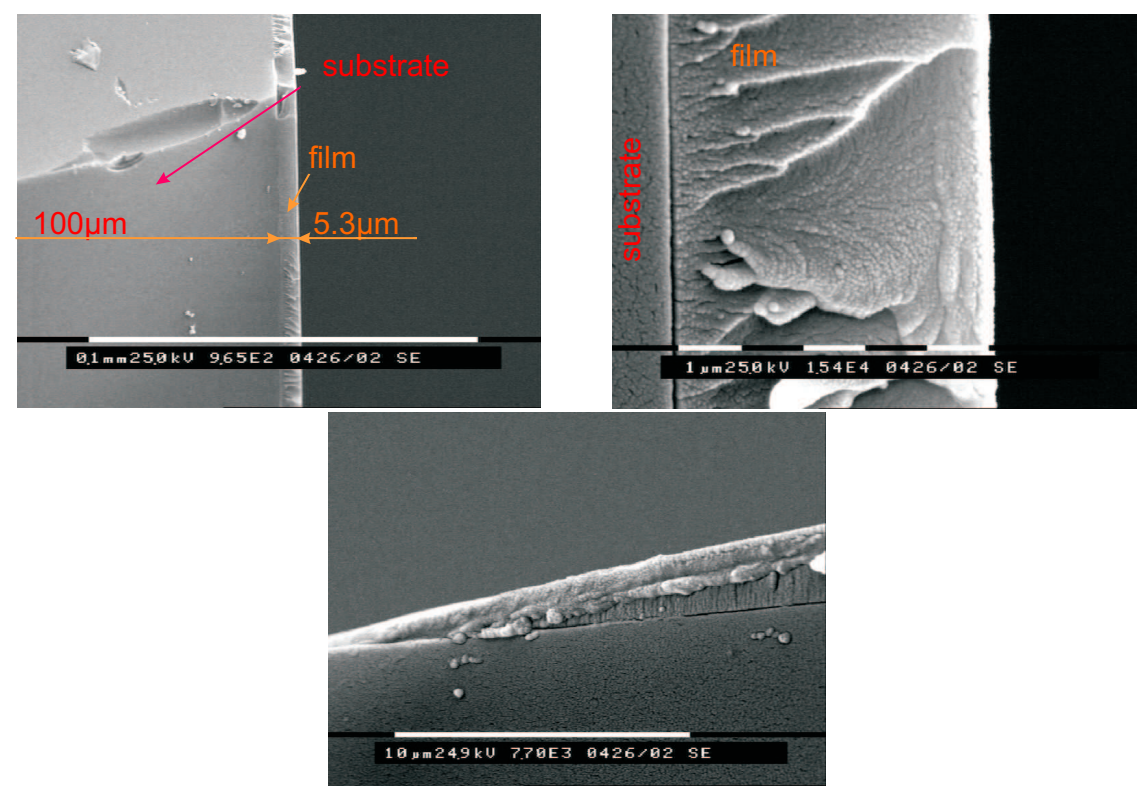

Figure 6.15: Cantilever and film broken after immersion in liquid nitrogen. The film surface and the film thickness can be analyzed by the scanning electron microscope (SEM). In the left hand side picture one can see that the film surface is smooth. The picture on the right hand side shows the ductile nature of the film. The lower picture depicts the curved edge of the film.

ness influences the peak height and not its position or relaxation broadness; therefore the exact value is not of high priority. With this analysis once again it is proved that the non-destructive, simple and cheap vibrating reed method can also be used for the determination of film thickness. 


\section{Chapter 7}

\section{Summary and outlook}

By means of mechanical spectroscopy the effect of reduced dimensions and increasing cross-linking degree on dynamics of polymer chains have been studied. Mechanical damping of supported polymer films was measured by the Vibrating Reed technique. In order to measure the internal friction in polymer films the damping of substrates had to be minimized. Therefore the substrates used in the experiments were made of $\mathrm{Si}(100)$ single crystals with optimized shape and dimensions to reduce losses. The method allows the study of the whole relaxation spectra near $T_{g}$.

The effect of reduced dimensions on the glass transition of supported polymer films was studied on the system Si-substrate/PS-film. The films were prepared by spin-coating a solution of PS in toluene. The experiments were performed with a low cooling/heating rate on polymers of molecular weight $M_{w}=10^{6}$ and the polydispersity index PDI=1.06.

For the films of thicknesses less than $100 \mathrm{~nm}$ the width of the $\alpha$ relaxation peak increases and shifts to the lower temperatures proving the increase in molecular mobility. However, below a certain thickness there was no further effect on the $\alpha$ relaxation peak observed. The most simple model explaining such a behavior is the existence of a liquid-like layer at the film/vacuum and the counteracting solid-like layer at the film/substrate interface. The molecules which extend to the liquid like layer can diffuse and that leads to the increase in mobility. The liquid-like layer responds in the viscous manner and hence it does not contribute to the internal friction. As a consequence if the films thickness reduces to the thickness of the liquid-like layer the glass transition temperature should approach zero. However, at the film/substrate interface the orientation of the phenyl rings out of the substrate causes the increase in density in the solid-like region close to the film/substrate interface. This leads to the inhibited molecular movements. At some film thickness the increase in the molecular mobility due the reptation equals the inhibited movements due to the increase in density. Within this work the depression in glass transition temperature was noticed for films 
of thicknesses between $44 \mathrm{~nm}$ and $95 \mathrm{~nm}$. The relaxation peaks of films of $26 \mathrm{~nm}$ and $7.5 \mathrm{~nm}$ are even slightly shifted to the higher temperatures, but these departures are within the uncertainties of the data.

The film thicknesses were varied by changing the concentration of solution and afterward determined from shifts in eigen frequencies of vibrating reeds. Results were compared to those observed by SEM. Despite the different geometries of vibrating reeds and the samples prepared for the SEM analysis, the thicknesses were comparable.

The further study on dynamics in thin polymer films should be performed on free standing polymer films. This could be accomplished by measuring the damping of vibrating membranes excited by an acoustic waves.

Another way to influence the dynamics in polymer films is to cross-link polymer chains. Within this work a system Si-substrate/PVCN-film was studied. The polymer is photosensitive and hence it was cross-linked by UV radiation. The degree of cross-linking was easily controlled by controlling the time of exposure to the UV light. The cross-links inhibit the molecular mobility leading to an increase in $T_{g}$ and a reduction of the relaxation strength. Below the $T_{g}$ the elastic modulus of the cross-linked structure increased about $50 \%$ and that caused a higher damping. The "base line" of the spectra shifts to the higher $Q^{-1}$ values due to the increase in modulus and probably due to the increase in the number of relaxation centers.

The cross-linking degree of the polymer was measured by the FT-IR as a decrease in a band attributed to the $\mathrm{C}=\mathrm{C}$ double bond which saturates during the cross-linking. The thickness was determined in the same way as in the case of the thin PS films.

After two hours exposure of the PVCN film to the UV light the $\alpha$ relaxation almost diminished although the cross-links density was only $80 \%$. In the IR spectra the cross-links density of $80 \%$ was hardly influenced by further UV exposure. The reason for the early structural arrest remains an opened question. Further investigations should include the study of diffusion of small molecules in cross-linked structure in order to prove the change in the density of PVCN films with cross-linking.

Dynamics of polymer films near $T_{g}$ was successfully investigated by means of mechanical spectroscopy. This investigation is the first successive study of the effect of reduced dimensions on dynamics in supported PS films. The vibrating reed method allows the measuring of dynamics in polymer films as well as a non-destructive determination of the film thickness from the shift in the eigen frequency of the vibrating reeds. Hence the method is a very good choice for investigation of dynamics depending on film thickness. 


\section{Appendix A}

\section{Energy-Dispersive X-Ray (EDX) Analysis of a Dry Etched Substrate.}

EDX analysis of the dry etched sample showed that after dry etching the surface of the reed is contaminated by $\mathrm{Fe}, \mathrm{Cr}, \mathrm{Ni}, \mathrm{Al}$ and $\mathrm{P}$ atoms. These atoms come from sputtering off the surface of the sample holder made of stainless steel.

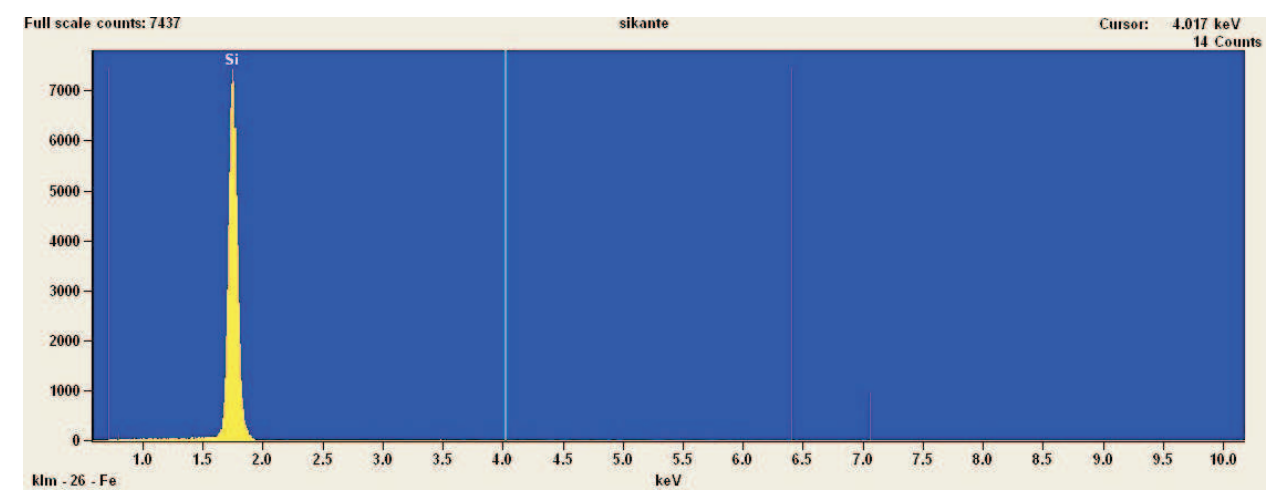

Figure A.1: The EDX scan of the dry etched Si substrate. 


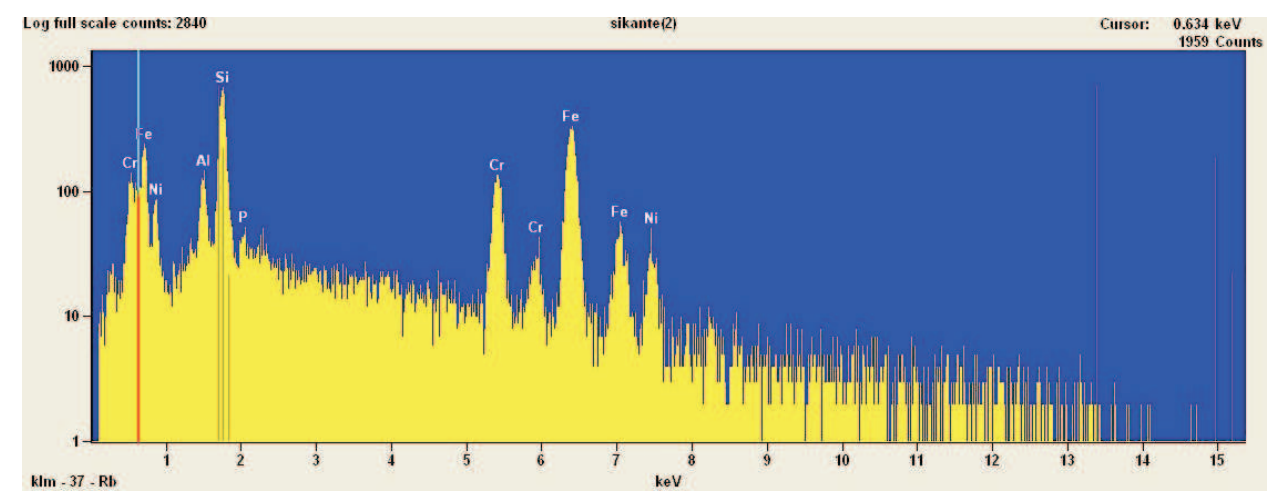

Figure A.2: The EDX scan of the dry etched Si substrate in a logarithmic scale reveals the $\mathrm{Fe}, \mathrm{Ni}$ and $\mathrm{Cr}$ atoms, belonging to the stainless steel of the sample holder. 


\section{Bibliography}

G. Adam and J. H. Gibbs, 1965, On the temperature dependence of cooperative relaxation properties in glass-forming liquids. The Journal of Chemical Physics 43, 139.

M. Alcoutlabi and G. McKenna, 2005, Effect of confinement on material behaviour at the nanometer size scale. Journal of Physics: Condensed Matter 17, R461.

C. A. Angell, 1988, Structural instability and relaxation in liquid and glassy phases near the fragile liquid limit. Journal of Non-Crystalline Solids 102, 205.

I. Bahar, N. Baysal, B. Erman and L. Monnerie, 1995, Kinematics of polymer chains in dense media. 3. Influence of intramolecular conformational potentials. Macromolecules 28, 1038.

D. P. Birnie III and M. Manley, 1997, Combined flow and evaporation of fluid on a spinning disk. Physics of Fluids 9, 870.

M. Buback, 2005. Private communication.

F. Bueche, 1953, Segmental mobility of polymers near their glass temperature. The Journal of Chemical Physics 21, 1850.

U. Buechenau, Glaszustand und glasübergang. In Physik der Polymere, edited by R. Hölzle, pp. 9.1-9.25 (Forschungszentrum Jülich, Insitutut für Festkörperforschung, 1991).

J. Y. Cavaille, L. David and J. Perez, Relaxation phenomena in non crystalline solids: Case of polymeric materials. In Mechanical Spectroscopy $Q^{-1} 2001$ with Applications to Materials Science, edited by R. Schaller, G. Fantozzi and G. Gremaud (Trans Tech Publications Ltd., Switzerland, 2001).

M. M. Coleman, M. S. Y. Hu and P. C. Painter, 1998, Infrared characterization of poly(vinyl cinnamate) and its blends with poly(4-vinyl phenol) before and after uv exposure. Journal of Polymer Science B 36, 1579. 
K. Dalnoki-Veress, J. Forrest, C. Murray, C. Gigault and J. R. Dutcher, 2001, Molecular weight dependence of reduction in the glass transition temperature of thin freely standing polymer films. Physical Review E 63, 031801.

P. de Gennes, 2000, Glass transitions in thin polymer films. Europian Physical Journal E 2, 201.

P. G. Debenedetti and F. Stillinger, 2001, Supercooled liquids and the glass transition. Nature 410, 259.

C. Decker, 2002, Light-induced crosslinking polymerization. Polymer International 51, 1141.

G. B. DeMaggio, W. E. Frieze, D. W. Gidley, M. Zhu, H. A. Hristov and A. F. Yee, 1997, Interface and surface effects on the glass transition in thin polystyrene films. Physical Review Letters 95, 025701.

R. O. Ebewele, Polymer Science and Technology (CRC Press LLC, Boca Raton, Florida, 2000).

M. Y. Efremov, E. A. Olson, M. Zhang, Z. Zhang and L. H. Allen, 2005, Glass transition in ultrathin polymer films: Calorimetric study. Physical Review Letters 95, 025701.

C. J. Ellison, S. D. Kim, D. B. Hall and J. M. Torkelson, 2002, Confinement and processing effects on glass transition temperature and physical aging in ultrathin polymer films. The European Physical Journal E 8, 155.

Z. Fakhraai and J. Forrest, 2005, Probing slow dynamics in supported thin polymer films. Physical Review Letters 95, 025701.

Z. Fakhraai, J. Sharp and J. Forrest, 2004, Effect of sample preparation on the glass-tansition of thin polystyrene films. Journal of Polymer Science: Part B:Polymer Physics 42, 4503.

Z. Fakhraai, S. Valadkhan and J. A. Forrest, 2005, Qualitative discrepancy between different measures of dynamics in thin polymer films. The European Physical Journal E 18, 143.

F. Faupel, R. Willecke and A. Thran, 1998, Diffusion of metals in polymers. Materials Science and Engineering: R: Report 22, 1.

J. Forrest and J. Sharp, 2003, Free surfaces cause reductions in the glass transition temperature of thin polystyrene films. Physical Review Letters 91, 235701.

J. A. Forrest and K. Dalnoki-Veress, 2001, The glass transition in thin polymer films. Advances in Colloid and Interface Science 94, 167. 
J. A. Forrest, K. Dalnoki-Veress, J. R. Stevens and J. R. Dutcher, 1996, Effect of free surfaces on the glass transition temperature of thin polymer films. Physical Review Letters 77, 2002.

K. Fukao, 2003, Dynamics in thin polymer films by dielectric spectroscopy. The European Physical Journal E 12, 119.

K. Fukao and Y. Miyamoto, 2000, Glass transition and dynamics in thin polymer films: Dielectric relaxation of thin films of polystyrene. Physical Review E 61, 1743.

K. Fukao and Y. Miyamoto, 2001, Slow dynamics near glass transition in thin polymer films. Physical Review E 64, 011803.

K. Fukao, S. Uno, Y. Miyamoto, A. Hoshino and H. Miyaji, 2001, Dynamics of $\alpha$ and $\beta$ processes in thin polymer films: Poly(vinyl acetate) and poly(methyl methacrylate). Physical Review E 64, 051807.

K. S. Gautam, A. D. Schwab, A. Dhinojwala, D. Zhang, S. M. Dougal and M. S. Yeganeh, 2000, Molecular structure of polystyrene at air/polymer and solid/polymer interfaces. Physical review letters 85, 3854 .

L. A. Giannuzzi and F. A. Stevie (Editors) Introduction to Focused Ion Beams, Instrumentation, Theory, Techniques and Practice (Springer Science+Business Media, Inc, New York, 2005).

E. J. Graeser and C. R. Wong, The relationship of traditional damping measures for materials with high damping capacity. In Mechanics and Mechanisms of Material Damping, edited by V. Kinra and A. Wolfenden, pp. 316-343 (ASTM STP 1196, Philadelphia, PA, 1992).

G. Grest, K. Kremer and E. Duering, 1992, Kinetics of end crosslinking in dense polymer melts. Europhysics Letters 19, 195.

J. Guillet, Polymer Photophysics and Photochemistry (Cambridge University Press, Cambridge, London, New York, New Rochelle, Melbourne, Sydney, 1985).

D. B. Hall, J. C. Hooker and J. M. Torkelson, 1997, Ultrathin polymer films near the glass transition: effect on the distribution of alpha-relaxation times as measured by second harmonic generation. Macromolecules 30, 667.

U. Harms, L. Kempen and H. Neuhauser, 1999, Vibrating reed apparatus with optical detection and digital signal processing: Application to measurements on thin films. Review of Scientific Instruments 70, 1751. 
L. Hartmann, W. Gorbatschow, J. Hauwede and F. Kremer, 2002, Molecular dynamics in thin films of isotactic poly(methyl methacrylate). The European Physical Journal E 8, 145.

M. Hasegawa, K. Kotani, S. Yamura, H. Kato, I. Kodama and A. Inoue, 2004, Hydrogen-induced internal friction of Zr-based bulk glassy alloys in a rod shape above 90 K. Journal of Alloys and Compounds 365, 221.

Y. Hiki and T. Kosugi, 2005, Relaxations in polystyrene below the glass transition studied by internal friction measurement. Journal of NonCrystalline Solids 351, 1300.

O. Hölck, 2000, Aufbau einer Vibrating-Reed-Apparatur zur Untersuchung der inneren Reibung kleiner Molecüle in polymeren Gläsern. Diploma thesis, Institute for Materials Physics, Göttingen.

R. Jones, Soft Condensed Matter (Oxford University Press, New York, 2003).

S. K. Kaliappan and B. Cappella, 2005, Temperature dependent elasticplastic behaviour of polystyrene studied using afm force-distance curves. Polymer 46, 11416.

S. Kawana and R. A. L. Jones, 2001, Character of the glass transition in thin supported polymer films. Physical Review E 63, 021501.

J. L. Keddie, R. A. L. Jones and R. A. Cory, 1994, Size-dependent depression of the glass transition temperature in polymer films. Europhysics Letters $\mathbf{2 7}, 59$.

H.-T. Kim and J.-K. Park, 1998, Thermal degradation of poly(vinyl cinnamate). Polymer Bulletin 41, 325.

L. E. Kinsler and A. R. Frey, Fundamentals of Acoustics. 2nd ed. (John Wiley and Sons, Inc., New York, London, 1962).

J. Kraus, P. Müller-Buschbaum, T. Kuhlmann, D. Schubert and T. Stamm, 2000, Confinement effect on the chain conformation in thin polymer films. Europhysics letters 49, 210.

D. R. Mason and A. P. Sutton, 2005, Computational steering in Monte Carlo simulations of thin film polystyrene. Philosophical transactions of the royal society A $\mathbf{3 6 3}, 1961$.

M. Matsuguchi, J. Miura and Y. Sakai, 2000, Effect of crosslinking structure on sorption of $\mathrm{CO}_{2}$ in photocrosslinked PVCA film. Journal of Applied Polymer Science 78, 1744. 
N. G. McCrum, B. E. Read and G. Williams, Anelastic and Dielectric Effects in Polymeric Solids (Dover Publications, Inc., New York, 1991).

G. B. McKenna, 2000, Size and confinement effects in glass forming liquids: perspectives on bulk and nano-scale behaviours. Journal de Physique IV 10,53 .

L. M. Minsk, J. Smith, W. V. Deusen and J. Wright, 1959, Photosensitive polymers. i. cinnamate esters of poly(vinyl alcohol) and cellulose. Journal of Applied Polymer Science 2, 302.

H. Mizubayashi, Y. Yoshihara and S. Okuda, 1992, The elasticity measurements of aluminium nm-films. Physica Status Solidi A 129, 475.

H. Neuhauser, A. Nagy, A. Strahl, H. N. I. Schrader, I. Behrens, E. Peiner and A. Schlachetzki, 2004, Mechanical spectroscopy of thin layers of ppv polymer on si substrates. Materials Science and Engineering A 370, 311.

C. E. Newell, 1968, Miniaturization of tuning forks. Science 161, 1320.

A. S. Nowick and B. S. Berry, Anelastic Relaxation in Crystalline Solids (Academic Press, New York and London, 1972).

J. Orloff, M. Utlaut and L. Swansono (Editors) High Resolution Focused Ion Beams: FIB and Its Applications; The Physics of Liquid Metal Ion Sources and Ion Optics and Their Application to Focused Ion Beam Tech (Kluwer Academic/Plenum Publishers, New York, Boston, Dordrecht, London, Moscow, 2005).

E. M. Robertson, W. P. V. Deusen and L. M. Minsk, 1959, Photosensitive polymers. ii. sensitization of poly(vinyl cinnamate). Journal of Applied Polymer Science 2, 308.

C. B. Roth and J. R. Dutcher, 1997, Interface and chain confinement effects on the glass transition temperature of thin polymer films. Physical Review E 56, 5705 .

C. B. Roth and J. R. Dutcher, 2003, Glass transition temperature of freelystanding films of atactic poly(methyl methacrylate). The European Physical Journal E 12, 103.

W. Runyan, Silicon Semiconductor Technology (McGraw-Hill, New York, 1965).

T. P. Russel, R. L. Jones, S. K. Kumar, D. L. Ho and R. B. Briber, 1999, Chain conformation in ultrathin polymer films. Nature 400, 146. 
J. San Juan, Mechanical spectroscopy. In Mechanical Spectroscopy $Q^{-1} 2001$ with Applications to Materials Science, edited by R. Schaller, G. Fantozzi and G. Gremaud (Trans Tech Publications Ltd., Switzerland, 2001).

A. Serghei, H. Huth, M. Schellenberger, C. Schick and F. Kremer, 2005, Pattern formation in thin polystyrene films induced by an enhanced mobility in ambient air. Physical Review E 71, 061801.

J. S. Sharp and J. A. Forrest, 2003, Dielectric and ellipsometric studies of the dynamics in thin films of isotactic poly(methylmethacrylate) with one free surface. Physical Review E 67, 031805.

J. S. Sharp, J. H. Teichroeb and J. A. Forrest, 2004, The properties of free polymer surfaces and their influence on the glass transition temperature of thin polystyrene films. The European Physical Journal E 15, 473.

L. Singh, P. J. Ludovice and C. L. Henderson, 2004, Influence of molecular weight and film thickness on the glass transition temperature and coefficient of thermal expansion of supported ultrathin polymer films. Thin Solid Films 449, 231.

T. Strunskus, V. Zaporojtchenko, K. Behnke, C. v. Bechtolsheim and F. Faupel, 2000, Tailoring the morphology of metal/polymer interfaces. Advanced Engineering Materials 22, 489.

E. Süske, 2005, Charakteristische Eigenschaften laserdeponierter PMMAFilme. Ph.D. thesis, Institute for Materials Physics, Göttingen.

H. Tanimoto, S. Sakai and H. Mizubayashi, 2004, Anelasticity of nanocrystalline metals. Materials Science and Engineering A 370, 135.

D. Van Krevelen, Properties of Polymers. 3rd ed. (Elsevier Science B.V., Amstedam, The Netherlands, 1990).

J. H. van Zanten, W. E. Wallace and W. Wu, 1996, Effect of strongly favorable substrate interactions on the thermal properties of ultrathin polymer films. Physical Review E 53, R2053.

M. Weller, Thin-layer materials. In Mechanical Spectroscopy $Q^{-1} 2001$ with Applications to Materials Science, edited by R. Schaller, G. Fantozzi and G. Gremaud (Trans Tech Publications Ltd., Switzerland, 2001).

O. Yaroshchuk, T. Sergan, J. Kelly and I. Gerus, 2002, Spatial anisotropy in poly(vinyl cinnamate) films. Japanese Journal of Applied Physics 41, 275.

G. Zener, 1937, Internal friction in solids. I. Theory of internal friction in reeds. Physical Review 52, 230. 



\section{Acknowledgment}

First, I would like to thank to Prof. Reiner Kirchheim for his generous supervision, stimulating discussions and his always optimistic attitude. Prof. Kirchheim opened me the door of physics and I am particularly thankful for that.

Many thanks to Prof. Konrad Samwer, my co-supervisor, for keeping his interest in the development of my work and his readiness to support me.

Special thanks to Dr. Hans-Georg Brion for many good advices in all possible scientific, technical and everyday's problems.

Ole Hölck deserves a special thanks for introducing me to the "secrets" of the Vibrating Reed Apparatus, many discussions, the exchange of literature and a very detailed reading through the manuscript. His attitudes, understanding and willingness to help all those who need it was always motivating.

I would also like to thank Prof. Hans-Ulrich Krebs for vivid humorous but scientific discussions and his effort in organizing informal meetings of students with similar scientific interests. The information exchange with Dr. Erik Süske, Throsten Scharf, Jörg Hachenberg, Dr. Peter Rösner and many others who participated was always a pleasure. The cooperation with Dr. Erik Süske resulted in a successful FT-IR study of cross-linked polymers.

I appreciate the support and help of Dr. Peter-Joachim Wilbrandt, Volker Radisch and Matthias Hahn in the FIB/SEM micrography.

Many thanks to all the technical support I received in the Institute, Karin Ahlborn, Mike Kanbach, Michael Malchow, Michael Hey, Denny Wagner and Herr Rothämel.

My thanks to PD Dr. Erwin Peiner and Arti Tibrewala from the Institute of Semiconductor Technology of the Technical University at Brunswick and Prof. Hiroshi Mizubayashi from the College of engineering sciences at the University of Tsukuba, Japan, for providing me with Si substrates.

I am very thankful to the DFG Graduate School, Spectroscopy and Dynamics of Molecular Aggregates, Chains and Coils, GRK 782, for the financial support, but also for helping in building a net of physicists and chemists who learnt the same scientific language. The opportunity to access many labs through many contacts resulted in lots of good advices and in possibility to make the SEC analysis of PS in the group of Prof. Buback at the 
Institute of Physical Chemistry.

A special thanks goes to all of them who made my stay at the Institute of Materials Physics a very pleasant experience: my roommates Ahmed Shariq and Carola Eyßell, our secretaries Christine Kuba, Frau Saalfeld, Frau Haake and all those who participated at the joyful coffee time.

My sincere thanks are given to my dearest husband Tomi Haramina whose curiosity brought us to Göttingen and who was always there when I needed him. 



\section{Lebenslauf}

\section{Persönliche Daten}

Name:

Geburtsdatum:

Geburtsort:

Staatsangehörigkeit:

\section{Schulbildung}

$07 / 1980-06 / 1988$

$07 / 1988-06 / 1992$

\section{Studium}

$10 / 1992-11 / 1999$

03/2002-07/2002

$12 / 2002-06 / 2006$

$12 / 2002-11 / 2005$

06/2006
Tatjana Haramina, geb. Sušić

15. Dezember 1973

Karlovac, Kroatien

kroatisch

Grundschule, Karlovac

Gymnasium, Karlovac; Abschluss: Abitur

Studium des Maschinenbaus an der Universität Zagreb, Fakultät für Maschinenbau und Schiffbau; Abschluss: Dipl. Ing. Maschinenbau Praktikum an der Universität Kassel, Institut für Werkstofftechnik, Kunststoff- und Recyclingtechnik Doktorarbeit betreut durch Prof. Dr. Reiner Kirchheim am Institut für Materialphysik der Universität Göttingen Stipendiatin des Graduirtenkolegs "GRK 782 Spektroskopie und Dynamik molekularer Aggregate, Ketten und Knäuel" Promotion in Physik an der Universität G̈̈tingen

\section{Berufliche Tätigkeit}

$10 / 2000-03 / 2002$

Softwareentwicklung bei "Siemens", Zagreb, Graz

$12 / 2005-08 / 2006$

Wissenschaftliche Mitarbeiterin an der Uni Göttingen, Institut für Materialphysik 